\title{
Ruthenium Catalyzed Intramolecular C-S coupling Reactions: Synthetic Scope and Mechanistic Insight
}

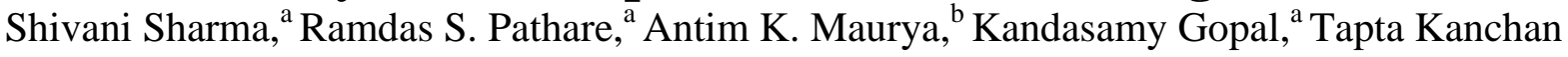
Roy, ${ }^{\text {a Devesh M. Sawant }}{ }^{\mathrm{b}^{*}}$ and Ram T. Pardasani ${ }^{\mathrm{a}}$

School of Chemical Sciences and Pharmacy, Central University of Rajasthan, Bandarsindri, Kishangarh, Ajmer, Rajasthan 305801, India

Email:dms@curaj.ac.in

\section{Contents}

Page no.

1. General considerations

S1

2. Detailed results of screening

S1

3. General procedure for the synthesis of compounds

4. Analytical data of benzothiazoles

S11

5. Kinetic isotope effect experiments

S39

6. Radical trap experiment

$\mathrm{S} 42$

7. Kinetic studies

$\mathrm{S} 42$

8. Crystallographic data

$\mathrm{S} 43$

9. Computational data

S45

10. References

S51 


\section{General Considerations}

All the reactions were carried out under argon atmosphere using standard Schlenk techniques. Anhydrous DCE was purchased from Sigma-Aldrich. N-arylthioureas 8 were readily prepared by reacting corresponding arylthiocyanates and amines ${ }^{1}$. All other reagents were purchased from Sigma Aldrich or Spectrochem and used as such without purification. Analytical TLC were performed using $2.5 \times 5 \mathrm{~cm}$ plate coated with $0.25 \mathrm{~mm}$ thickness of silica gel $(60 \mathrm{~F}-254 \mathrm{Merck})$ and visualization was accomplished with $\mathrm{UV}$ light or $\mathrm{I}_{2} / \mathrm{KMnO}_{4}$ staining. ${ }^{1} \mathrm{H}$ and ${ }^{13} \mathrm{C} \mathrm{NMR}$ spectra were obtained from Bruker's Ascend $500 \mathrm{MHz}$ spectrophotometer operating at 500.3 $\mathrm{MHz}$ for ${ }^{1} \mathrm{H}$ and $125.8 \mathrm{MHz}$ for ${ }^{13} \mathrm{C}$ experiments. The chemical shifts are reported in ppm scale with respect to $\mathrm{CDCl}_{3}(7.269 \mathrm{ppm})$ for ${ }^{1} \mathrm{H}$ and $(77.00 \mathrm{ppm})$ for ${ }^{13} \mathrm{C} \mathrm{NMR}$ as internal standard.The abbreviations are used: $\mathrm{s}=$ singlet, $\mathrm{d}=$ doublet, $\mathrm{t}=$ triplet, $\mathrm{q}=$ quartet, $\mathrm{dd}=$ double doublet, $\mathrm{m}=$ multiplet, brs $=$ broad singlet $\&$ br $=$ broad signal. Melting Points were recorded on Buchi M-655 Melting point apparatus and are uncorrected. Kinetic studies were performed on HPLC system LC-2010CHT (M/s Shimadzu Co Ltd Tokya Japan) using C-18 RP HPLC column (C18G, $250 \mathrm{x}$ $4.6 \mathrm{~mm}, 5 \mu \mathrm{m}$, Spinco Biotech Pvt Ltd, Mumbai, India). The mobile phase consisted of water and acetonitrile in 40:60 v/v ratio at a flow rate of $1.0 \mathrm{~mL} / \mathrm{min}$ at the scanning wavelength of $254 \mathrm{~nm}$. High resolution mass spectra (ESI-HRMS) were measured with a Waters GCT PremierCAB155 instrument and accurate masses were reported for the molecular ion $\left(\mathrm{M}^{+}\right)$. All the computational analysis has been carried out using Gaussian 09 program suit.

\section{Detailed Results of Screening}

\subsection{Table S1: Initial Screening}

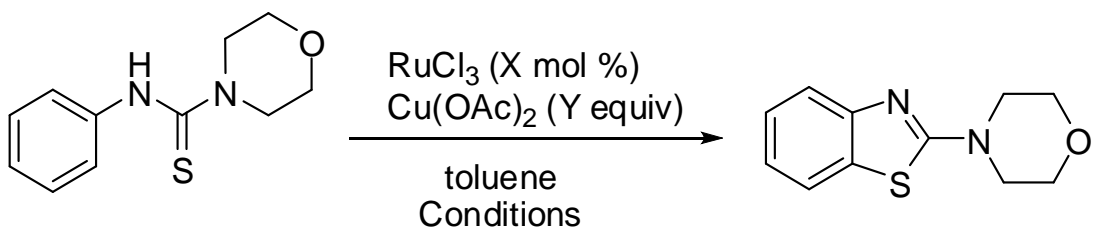

\begin{tabular}{ccccc}
\hline S.No. & $\mathrm{X}$ & $\mathrm{Y}$ & Conditions & Isolated yield (\%) \\
\hline $\mathbf{1 .}$ & $\mathbf{1 0}$ & $\mathbf{1}$ & $\mathbf{1 1 0}{ }^{\circ} \mathbf{C}, \mathbf{2 4} \mathbf{h}$ & $\mathbf{6 1}$ \\
2. & 10 & 2 & $110^{\circ} \mathrm{C}, 24 \mathrm{~h}$ & 54 \\
3. & 10 & 1 & $110^{\circ} \mathrm{C}, 36 \mathrm{~h}$ & 68 \\
4. & 10 & 1 & $70^{\circ} \mathrm{C}, 36 \mathrm{~h}$ & 43 \\
5. & 10 & 1 & $90{ }^{\circ} \mathrm{C}, 36 \mathrm{~h}$ & 48 \\
6. & 10 & 0 & $110{ }^{\circ} \mathrm{C}, 36 \mathrm{~h}$ & 18 \\
7. & 0 & 1 & $110^{\circ} \mathrm{C}, 36 \mathrm{~h}$ & 10 \\
\hline
\end{tabular}

\subsection{Table S2: Oxidant effect}

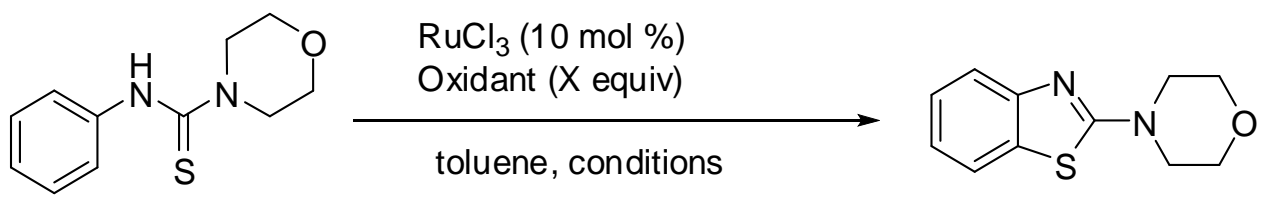




\begin{tabular}{ccccc}
\hline S.No. & Oxidant & $\mathrm{X}$ & Conditions & Isolated yield (\%) \\
\hline 1. & $\mathrm{~K}_{2} \mathrm{~S}_{2} \mathrm{O}_{8}$ & 1 & $110{ }^{\circ} \mathrm{C}, 24 \mathrm{~h}$ & 59 \\
2. & $\mathrm{NH}_{4} \mathrm{~S}_{2} \mathrm{O}_{8}$ & 1 & $110{ }^{\circ} \mathrm{C}, 24 \mathrm{~h}$ & 63 \\
3. & $\mathrm{DDQ}$ & 1 & $110{ }^{\circ} \mathrm{C}, 48 \mathrm{~h}$ & 28 \\
4. & $\mathrm{BQ}$ & 1 & $110{ }^{\circ} \mathrm{C}, 48 \mathrm{~h}$ & 35 \\
5. & $\mathrm{Cu}(\mathrm{OAc})_{2}$ & 1 & $110{ }^{\circ} \mathrm{C}, 24 \mathrm{~h}$ & 61 \\
6. & $\mathrm{AgOAc}$ & 1 & $110{ }^{\circ} \mathrm{C}, 48 \mathrm{~h}$ & 25 \\
7. & $\mathrm{CuI}$ & 1 & $110{ }^{\circ} \mathrm{C}, 24 \mathrm{~h}$ & 69 \\
8. & Oxone & 2 & $110{ }^{\circ} \mathrm{C}, 24 \mathrm{~h}$ & $\mathbf{8 0}$ \\
9. & $\mathrm{Oxone}$ & 1 & $110{ }^{\circ} \mathrm{C}, 24 \mathrm{~h}$ & 67 \\
10. & $\mathrm{Oxone}$ & 3 & $110{ }^{\circ} \mathrm{C}, 24 \mathrm{~h}$ & 81 \\
11. & ${ }^{t} \mathrm{BuOOH}$ & 1 & $110{ }^{\circ} \mathrm{C}, 48 \mathrm{~h}$ & 0 \\
12. & - & - & $110{ }^{\circ} \mathrm{C}, 48 \mathrm{~h}$ & 18 \\
\hline
\end{tabular}

\subsection{Table S3: Solvent effect}

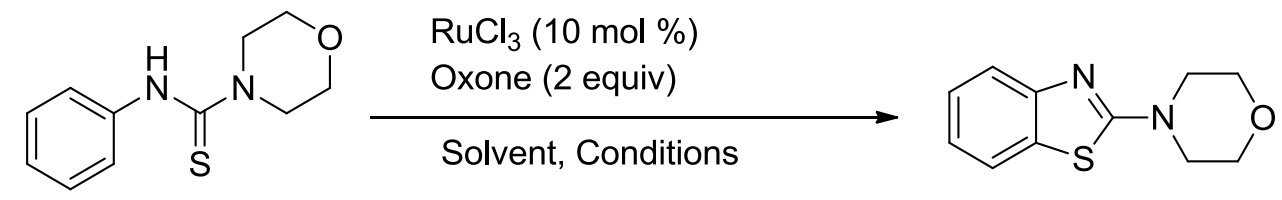

\begin{tabular}{cccc}
\hline S.No. & Solvent & Conditions & Isolated yield (\%) \\
\hline 1 & toluene & $110^{\circ} \mathrm{C}, 24 \mathrm{~h}$ & 80 \\
2 & THF & $80^{\circ} \mathrm{C}, 48 \mathrm{~h}$ & 75 \\
3 & dioxane & $110^{\circ} \mathrm{C}, 48 \mathrm{~h}$ & 38 \\
4 & DCM & $50^{\circ} \mathrm{C}, 48 \mathrm{~h}$ & 27 \\
$\mathbf{5}$ & DCE & $\mathbf{1 0 0}^{\circ} \mathbf{C}, \mathbf{6 ~ h}$ & $\mathbf{8 9}$ \\
6 & xylene & $110^{\circ} \mathrm{C}, 48 \mathrm{~h}$ & 65 \\
7 & $\mathrm{DMF}$ & $110^{\circ} \mathrm{C}, 48 \mathrm{~h}$ & 12 \\
8 & $\mathrm{DMSO}$ & $110^{\circ} \mathrm{C}, 48 \mathrm{~h}$ & 57 \\
9 & $\mathrm{H}_{2} \mathrm{O}$ & $110^{\circ} \mathrm{C}, 48 \mathrm{~h}$ & 35 \\
10 & $\mathrm{CH}_{3} \mathrm{COOH}$ & $120^{\circ} \mathrm{C}, 48 \mathrm{~h}$ & 15 \\
11 & $\mathrm{CH}_{3} \mathrm{CN}$ & $90^{\circ} \mathrm{C}, 48 \mathrm{~h}$ & 20 \\
12 & $\mathrm{EtOH}$ & $85^{\circ} \mathrm{C}, 48 \mathrm{~h}$ & 40 \\
\hline
\end{tabular}

\subsection{Table S4: Ruthenium source and ligand effect}
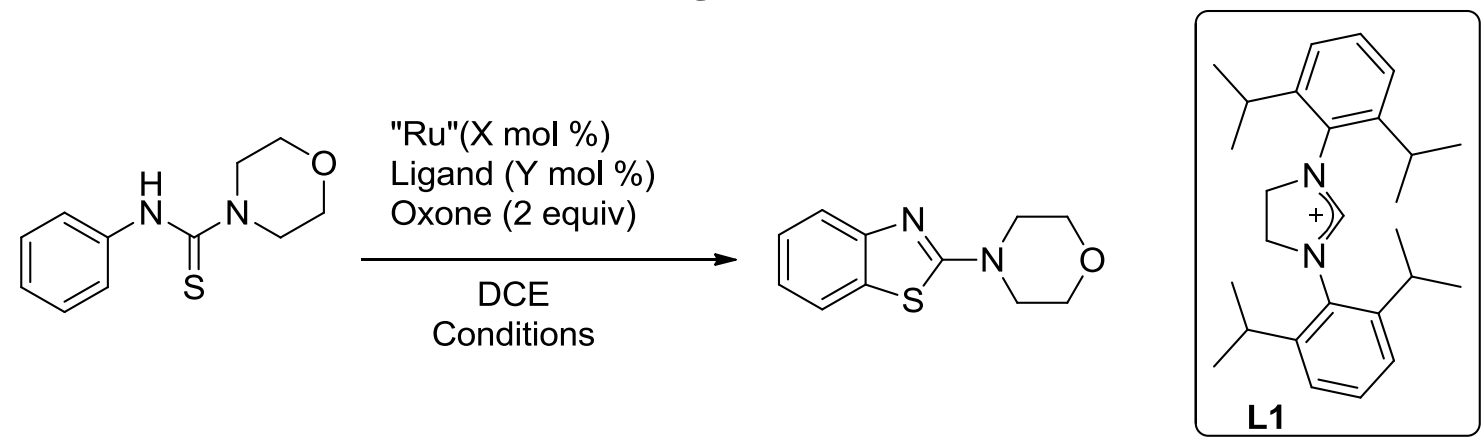


\begin{tabular}{|c|c|c|c|c|c|c|}
\hline & & & & & & Yield $(\%)$ \\
\hline 1. & $\mathrm{RuCl}_{3}$ & 10 & - & - & $100{ }^{\circ} \mathrm{C}, 24 \mathrm{~h}$ & 89 \\
\hline 2. & {$\left[\mathrm{RuCp} * \mathrm{Cl}_{2}\right]_{2}$} & 10 & - & - & $100^{\circ} \mathrm{C}, 48 \mathrm{~h}$ & 37 \\
\hline 3. & $\mathrm{RuCl}_{3}$ & 10 & $\mathrm{PPh}_{3}$ & 20 & $100^{\circ} \mathrm{C}, 48 \mathrm{~h}$ & 80 \\
\hline 4. & $\mathrm{RuCl}_{3}$ & 10 & Xantphos & 20 & $100{ }^{\circ} \mathrm{C}, 48 \mathrm{~h}$ & 78 \\
\hline 5. & $\mathrm{RuCl}_{3}$ & 10 & dppf & 20 & $100{ }^{\circ} \mathrm{C}, 48 \mathrm{~h}$ & 65 \\
\hline 6. & $\mathrm{RuCl}_{3}$ & 10 & $\mathbf{L} \mathbf{1}^{\mathrm{b}}$ & 30 & $120^{\circ} \mathrm{C}, 24 \mathrm{~h}$ & 77 \\
\hline 7. & $\mathrm{RuCl}_{3}$ & 10 & 2,2'-bipyridine & 30 & $100^{\circ} \mathrm{C}, 48 \mathrm{~h}$ & 54 \\
\hline 8. & {$\left[\mathrm{RuCl}_{2}(p \text {-cymene })\right]_{2}^{\mathrm{b}}$} & 10 & $\mathrm{PPh}_{3}$ & 20 & $100^{\circ} \mathrm{C}, 48 \mathrm{~h}$ & 78 \\
\hline 9. & {$\left[\mathrm{RuCl}_{2}(p \text {-cymene })\right]_{2}^{\mathrm{b}}$} & 10 & Xantphos & 20 & $100^{\circ} \mathrm{C}, 48 \mathrm{~h}$ & 70 \\
\hline 10. & {$\left[\mathrm{RuCl}_{2}(p \text {-cymene })\right]_{2}^{\mathrm{b}}$} & 10 & dppf & 20 & $100^{\circ} \mathrm{C}, 48 \mathrm{~h}$ & 65 \\
\hline 11. & {$\left[\mathrm{RuCl}_{2}(p \text {-cymene })\right]_{2}^{\mathrm{b}}$} & 10 & $\mathbf{L} \mathbf{1}^{\mathrm{c}}$ & 30 & $120^{\circ} \mathrm{C}, 24 \mathrm{~h}$ & 72 \\
\hline 12. & {$\left[\mathrm{RuCl}_{2}(p \text {-cymene })\right]_{2}$} & 10 & 2,2'-bipyridine & 30 & $100^{\circ} \mathrm{C}, 48 \mathrm{~h}$ & 64 \\
\hline 13. & {$\left[\mathrm{RuCl}_{2}(p \text {-cymene })\right]_{2}^{\mathrm{b}}$} & 10 & - & - & $100^{\circ} \mathrm{C}, 6 \mathrm{~h}$ & 82 \\
\hline
\end{tabular}

${ }^{a} \mathrm{NHC}$ was generated by reacting $\mathrm{Ll}$ with $\mathrm{Cs}_{2} \mathrm{CO}_{3}$ as reported earlier; ${ }^{2}{ }^{b} \mathrm{AgSbF}$ used to activate the catalyst.

Protocol for generation of Ru-NHC complex ${ }^{1}{ }^{a} N$-phenylmorpholine-4-carbothioamide (1.00 $\mathrm{mmol})$ were added to a solution of $\left[\mathrm{RuCl}_{2}\right.$ (p-cymene $\left.)\right]_{2}(0.10 \mathrm{mmol}), \mathbf{L 1}(0.30 \mathrm{mmol})$ and $\mathrm{Cs}_{2} \mathrm{CO}_{3}(3.00 \mathrm{mmol})$ in $N$-Methylpyrrolidone(NMP) $(2 \mathrm{~mL})$. The resulting mixture was stirred at $120{ }^{\circ} \mathrm{C}$ for $24 \mathrm{~h}$.

2.5 Table S5: Final optimization with $\mathrm{RuCl}_{3}$ and $\left[\mathrm{RuCl}_{2}(p \text {-cymene) }]_{2}\right.$

\begin{tabular}{|c|c|c|c|c|c|}
\hline S.No. & Catalyst & $\mathrm{X}$ & $\bar{Y}$ & Conditions & Yield (\%) \\
\hline 1. & $\mathrm{RuCl}_{3}$ & 10 & 2 & $100{ }^{\circ} \mathrm{C}, 6 \mathrm{~h}$ & 89 \\
\hline 2. & $\mathbf{R u C l}_{3}$ & 5 & 2 & $100^{\circ} \mathrm{C}, 6 \mathrm{~h}$ & 87 \\
\hline 3. & $\mathrm{RuCl}_{3}$ & 2.5 & 2 & $100{ }^{\circ} \mathrm{C}, 6 \mathrm{~h}$ & 80 \\
\hline 4. & $\mathrm{RuCl}_{3}$ & 1 & 2 & $100{ }^{\circ} \mathrm{C}, 6 \mathrm{~h}$ & 50 \\
\hline 5. & $\mathrm{RuCl}_{3}$ & 5 & 3 & $100{ }^{\circ} \mathrm{C}, 24 \mathrm{~h}$ & 89 \\
\hline 6. & $\mathrm{RuCl}_{3}$ & 5 & 1 & $100{ }^{\circ} \mathrm{C}, 24 \mathrm{~h}$ & 67 \\
\hline 7. & $\mathrm{RuCl}_{3}$ & 5 & 2 & $60^{\circ} \mathrm{C}, 24 \mathrm{~h}$ & 45 \\
\hline 8. & $\mathrm{RuCl}_{3}$ & 5 & 2 & $80^{\circ} \mathrm{C}, 24 \mathrm{~h}$ & 70 \\
\hline 9. & - & - & 2 & $100{ }^{\circ} \mathrm{C}, 24 \mathrm{~h}$ & 15 \\
\hline 10. & $\mathrm{RuCl}_{3}$ & 5 & 0 & $100{ }^{\circ} \mathrm{C}, 24 \mathrm{~h}$ & 12 \\
\hline 11. & {$\left[\operatorname{RuCl}_{2}(p \text {-cymene })\right]_{2}{ }^{\mathrm{a}}$} & 5 & 2 & $100^{\circ} \mathrm{C}, 6 \mathrm{~h}$ & 80 \\
\hline 12. & {$\left[\mathrm{RuCl}_{2}(p \text {-cymene })\right]_{2}^{\mathrm{a}}$} & 10 & 2 & $100{ }^{\circ} \mathrm{C}, 6 \mathrm{~h}$ & 82 \\
\hline 13. & {$\left[\mathrm{RuCl}_{2}(p \text {-cymene })\right]_{2}^{\mathrm{a}}$} & 5 & 2 & $60^{\circ} \mathrm{C}, 24 \mathrm{~h}$ & 48 \\
\hline 14. & {$\left[\mathrm{RuCl}_{2}(p \text {-cymene })\right]_{2}^{\mathrm{a}}$} & 5 & 2 & $80^{\circ} \mathrm{C}, 24 \mathrm{~h}$ & 64 \\
\hline 15. & - & - & 2 & $100{ }^{\circ} \mathrm{C}, 6 \mathrm{~h}$ & 15 \\
\hline 16. & {$\left[\mathrm{RuCl}_{2}(p \text {-cymene })\right]_{2}^{\mathrm{b}}$} & 5 & 0 & $100{ }^{\circ} \mathrm{C}, 6 \mathrm{~h}$ & 20 \\
\hline
\end{tabular}

${ }^{a} \mathrm{AgSbF} \mathrm{F}_{6}$ used to activate the catalyst. 
2.6 Table S6: Screening of Catalyst

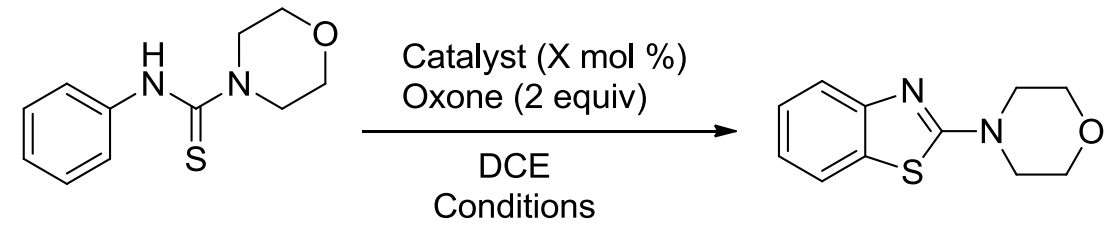

\begin{tabular}{ccccc}
\hline S.No. & Catalyst & $\mathrm{X}$ & Conditions & $\begin{array}{c}\text { Isolated Yield } \\
(\%)\end{array}$ \\
\hline 1. & $\mathrm{PdCl}_{2}$ & 5 & $80{ }^{\circ} \mathrm{C}, 6 \mathrm{~h}$ & 91 \\
2. & $\mathrm{Pd}(\mathrm{OAc})_{2}$ & 5 & $80{ }^{\circ} \mathrm{C}, 6 \mathrm{~h}$ & 92 \\
3. & {$\left[\mathrm{RuCl}_{2}(p-\mathrm{cymene})\right]_{2}$} & 5 & $100^{\circ} \mathrm{C}, 6 \mathrm{~h}$ & 80 \\
4. & $\mathrm{RhCl}_{3}$ & 10 & $100^{\circ} \mathrm{C}, 24 \mathrm{~h}$ & 0 \\
5. & {$\left[\mathrm{RhCp}^{*} \mathrm{Cl}_{2}\right]_{2}$} & 10 & $100^{\circ} \mathrm{C}, 24 \mathrm{~h}$ & 0 \\
6. & $\mathrm{FeCl}_{3}$ & 10 & $100^{\circ} \mathrm{C}, 24 \mathrm{~h}$ & 12 \\
7. & $\mathrm{NiCl}_{2}$ & 10 & $100^{\circ} \mathrm{C}, 24 \mathrm{~h}$ & 0 \\
8. & $\mathrm{AuCl}$ & 10 & $100^{\circ} \mathrm{C}, 24 \mathrm{~h}$ & 0 \\
\hline
\end{tabular}

3. General procedure for the synthesis of compounds 9a-v:

A schlenk tube equipped with a stir-bar was charged with thiourea. Then DCE was added to the reaction tube via a syringe. The reaction tube was purged with argon. Then after 5-10 min, Oxone and $\mathrm{RuCl}_{3}$ was added to the reaction mixture followed by purging of argon gas. Subsequently, argon was replaced by air and the reaction mixture was stirred at $110{ }^{\circ} \mathrm{C}$ for $4-10$ h. On the completion of the reaction on TLC, the reaction mixture was passed through celite bed and concentrated under reduced pressure and then purified by silica gel chromatography (EtOAc:petroleum ether) to yield the desired product. The specific reaction condition and characterization data for each reaction are given below.

\section{9a: 4-(Benzo[d]thiazol-2-yl)morpholine}

$N$-Phenylmorpholine-4-carbothioamide $(0.05 \mathrm{~g}, 0.224 \mathrm{mmol})$, Oxone $(0.138 \mathrm{~g}, 0.448 \mathrm{mmol}), \mathrm{RuCl}_{3}$ $(0.0023 \mathrm{~g}, 0.012 \mathrm{mmol})$, DCE $(3 \mathrm{~mL})$. Product: golden brown solid, yield: $0.044 \mathrm{~g}(87 \%)$; m.p.:118-119 ${ }^{\circ} \mathrm{C}\left[\right.$ lit. $\left.^{3} 119-120{ }^{\circ} \mathrm{C}\right] .{ }^{1} \mathrm{H}$ NMR $(\delta \mathrm{ppm}):\left(500 \mathrm{MHz}, \mathrm{CDCl}_{3}\right), 7.62(\mathrm{dd}, 1 \mathrm{H}$, aromatic C- $H, J=0.5,8 \mathrm{~Hz}), 7.58(\mathrm{~d}, 1 \mathrm{H}$, aromatic $\mathrm{C}-H, J=8 \mathrm{~Hz}), 7.32(\mathrm{dt}, 1 \mathrm{H}$, aromatic C- $H, J$ $=1.0,8.5 \mathrm{~Hz}), 7.12(\mathrm{dt}, 1 \mathrm{H}$, aromatic $\mathrm{C}-H, J=1.0,8.0 \mathrm{~Hz}), 3.84\left(\mathrm{t}, 4 \mathrm{H}, \mathrm{sp}^{3} \mathrm{C}-H, J=4.9 \mathrm{~Hz}\right)$, 3.63 (t, $\left.4 \mathrm{H}, \mathrm{sp}^{3} \mathrm{C}-H, J=4.9 \mathrm{~Hz}\right) .{ }^{13} \mathrm{C} \mathrm{NMR}(\delta \mathrm{ppm}):\left(125 \mathrm{MHz}, \mathrm{CDCl}_{3}\right): 169.05,152.49$, $130.58,126.13,121.72,120.81,119.33,66.26,48.51$. HRMS (EI) calcd for $\mathrm{C}_{11} \mathrm{H}_{12} \mathrm{~N}_{2} \mathrm{OS}\left(\mathrm{M}^{+}\right)$ 220.0670 , found 220.0679 . 


\section{9b: 4-(6-Ethylbenzo[d]thiazol-2-yl)morpholine}

$N$-(4-Ethylphenyl)morpholine-4-carbothioamide (0.05 g, $0.199 \mathrm{mmol})$, Oxone (0.061 g, 0.398 $\mathrm{mmol}), \mathrm{RuCl}_{3}(0.0018 \mathrm{~g}, 0.009 \mathrm{mmol}), \mathrm{DCE}(3 \mathrm{~mL})$. Product: brown solid, yield: $0.035 \mathrm{~g}(71 \%)$; m.p.: $100-102{ }^{\circ} \mathrm{C} .{ }^{1} \mathrm{H}$ NMR $(\delta \mathrm{ppm}):\left(500 \mathrm{MHz}, \mathrm{CDCl}_{3}\right), 7.49(\mathrm{~d}, 1 \mathrm{H}$, aromatic $\mathrm{C}-H, J=8.5 \mathrm{~Hz})$, $7.46(\mathrm{~d}, 1 \mathrm{H}$, aromatic C- $H, J=1.5 \mathrm{~Hz}), 7.16(\mathrm{dd}, 1 \mathrm{H}$, aromatic $\mathrm{C}-H, J=1.5,8 \mathrm{~Hz}), 3.84(\mathrm{t}, 4 \mathrm{H}$, $\left.\mathrm{sp}^{3} \mathrm{C}-H, J=4.7 \mathrm{~Hz}\right), 3.61\left(\mathrm{t}, 4 \mathrm{H}, \mathrm{sp}^{3} \mathrm{C}-H, J=4.7 \mathrm{~Hz}\right), 2.70\left(\mathrm{q}, 2 \mathrm{H}, \mathrm{sp}^{3} \mathrm{C}-\mathrm{H}, J=7.5 \mathrm{~Hz}\right), 1.26(\mathrm{t}$, $\left.3 \mathrm{H} ., \mathrm{sp}^{3} \mathrm{C}-H, J=7.5 \mathrm{~Hz}\right),{ }^{13} \mathrm{C} \mathrm{NMR}(\delta \mathrm{ppm}):\left(125 \mathrm{MHz}, \mathrm{CDCl}_{3}\right): 168.63,150.44,138.11,130.63$, $126.25,119.70,119.03,66.28,48.53,28.73,16.02$. HRMS (EI) calcd for $\mathrm{C}_{13} \mathrm{H}_{16} \mathrm{~N}_{2} \mathrm{OS}\left(\mathrm{M}^{+}\right)$ 248.0983 , found 248.0987 .

\section{9c: 4-(6-Methoxybenzo[d]thiazol-2-yl)morpholine}

$N$-(4-Methoxyphenyl)morpholine-4-carbothioamide $\quad(0.05 \mathrm{~g}, \quad 0.198 \mathrm{mmol}), \quad$ Oxone $\quad(0.122 \mathrm{~g}$, $0.396 \mathrm{mmol}), \mathrm{RuCl}_{3}(0.002 \mathrm{~g}, 0.01 \mathrm{mmol}), \mathrm{DCE}(3 \mathrm{~mL})$. Product: brick brown solid, yield: 0.042 $\mathrm{g}(85 \%)$;m.p.: $137-138{ }^{\circ} \mathrm{C}$ [lit. $\left.{ }^{3} 137-138{ }^{\circ} \mathrm{C}\right] .{ }^{1} \mathrm{H} \mathrm{NMR}(\delta \mathrm{ppm}):\left(500 \mathrm{MHz}, \mathrm{CDCl}_{3}\right), 7.48(\mathrm{dd}, 1 \mathrm{H}$, aromatic C- $H, J=3.5,9 \mathrm{~Hz}), 7.16(\mathrm{t}, 1 \mathrm{H}$, aromatic C- $H, J=3 \mathrm{~Hz}), 6.92(\mathrm{dd}, 1 \mathrm{H}$, aromatic C- $H$, $J=2.5,8.8 \mathrm{~Hz}), 3.85-3.83\left(\mathrm{~m}, 4 \mathrm{H}, \mathrm{sp}^{3} \mathrm{C}-H\right), 3.83\left(\mathrm{~s}, 3 \mathrm{H}, \mathrm{OCH}_{3}\right), 3.58\left(\mathrm{t}, 4 \mathrm{H}, \mathrm{sp}^{3} \mathrm{C}-H, J=4.75\right.$ $\mathrm{Hz}) .{ }^{13} \mathrm{C}$ NMR $(\delta \mathrm{ppm}):\left(125 \mathrm{MHz}, \mathrm{CDCl}_{3}\right): 167.70,155.22,146.64,131.59,119.79,113.79$, 105.22, 66.28, 55.85, 48.53. HRMS (EI) calcd for $\mathrm{C}_{12} \mathrm{H}_{14} \mathrm{~N}_{2} \mathrm{O}_{2} \mathrm{~S}\left(\mathrm{M}^{+}\right)$250.0776, found 250.0767.

\section{9d: 4-(6-Nitrobenzo[d]thiazol-2-yl)morpholine}

$\mathrm{N}$-(4-Nitrophenyl)morpholine-4-carbothioamide (0.05 g, $0.187 \mathrm{mmol})$, Oxone $(0.115 \mathrm{~g}, 0.374$ $\mathrm{mmol}), \mathrm{RuCl}_{3}(0.0018 \mathrm{~g}, 0.009 \mathrm{mmol}), \mathrm{DCE}$ (3 mL). Product: yellowsolid,yield: $0.039 \mathrm{~g}(79 \%)$; m.p.: decompose $>200{ }^{\circ} \mathrm{C}\left[\right.$ lit. $^{3}$ decompose $\left.>200{ }^{\circ} \mathrm{C}\right] .{ }^{1} \mathrm{H}$ NMR $(\delta \mathrm{ppm}):\left(500 \mathrm{MHz}, \mathrm{CDCl}_{3}\right), 8.51$ $(\mathrm{d}, 1 \mathrm{H}$, aromatic C- $H, J=2.4 \mathrm{~Hz}), 8.20(\mathrm{dd}, 1 \mathrm{H}$, aromatic C- $H, J=2.5,9 \mathrm{~Hz}), 7.51(\mathrm{~d}, 1 \mathrm{H}$, aromatic C- $H, J=9 \mathrm{~Hz}), 3.86\left(\mathrm{t}, 4 \mathrm{H}, \mathrm{sp}^{3} \mathrm{C}-H, J=4.9 \mathrm{~Hz}\right), 3.71\left(\mathrm{t}, 4 \mathrm{H}, \mathrm{sp}^{3} \mathrm{C}-H, J=4.8 \mathrm{~Hz}\right) .{ }^{13} \mathrm{C}$ NMR $(\delta \mathrm{ppm}):\left(125 \mathrm{MHz}, \mathrm{CDCl}_{3}\right): 172.03,157.87,141.88,130.76,122.65,118.39,117.37$, 66.15, 48.59. HRMS (EI) calcd for $\mathrm{C}_{11} \mathrm{H}_{11} \mathrm{~N}_{3} \mathrm{O}_{3} \mathrm{~S}\left(\mathrm{M}^{+}\right)$265.0521, found 265.0526.

\section{9e: 4-(6-Fluorobenzo[d]thiazol-2-yl)morpholine}

$N$-(4-Fluorophenyl)morpholine-4-carbothioamide (0.05 g, 0.208mmol), Oxone (0.128 g, 0.416 $\mathrm{mmol}), \mathrm{RuCl}_{3}(0.002 \mathrm{~g}, 0.01 \mathrm{mmol})$, DCE $(3 \mathrm{~mL})$. Product: brick brown solid, yield: $0.037 \mathrm{~g}$ (75\%); m.p.: 146-147 ${ }^{\circ} \mathrm{C}$ [lit. $\left.{ }^{3} 146-147.5{ }^{\circ} \mathrm{C}\right] .{ }^{1} \mathrm{H}$ NMR $(\delta \mathrm{ppm}):\left(500 \mathrm{MHz}, \mathrm{CDCl}_{3}\right), 7.49$ (dd, $1 \mathrm{H}$, aromatic $\mathrm{C}-H, J=4.5,8.5 \mathrm{~Hz}), 7.34(\mathrm{dd}, 1 \mathrm{H}$, aromatic $\mathrm{C}-H, J=2.5,8 \mathrm{~Hz}), 7.04(\mathrm{ddd}, 1 \mathrm{H}$, aromatic C- $H, J=2.5,9 \mathrm{~Hz}), 3.84\left(\mathrm{t}, 4 \mathrm{H}, \mathrm{sp}^{3} \mathrm{C}-H, J=5 \mathrm{~Hz}\right), 3.60\left(\mathrm{t}, 4 \mathrm{H}, \mathrm{sp}^{3} \mathrm{C}-H, J=5 \mathrm{~Hz}\right)$. ${ }^{13} \mathrm{C}$ NMR $(\delta \mathrm{ppm}):\left(125 \mathrm{MHz}, \mathrm{CDCl}_{3}\right): 168.64,158.26\left(J_{\mathrm{C}-\mathrm{F}}=239 \mathrm{~Hz}\right), 148.92,131.23,119.78$ $\left(J_{\mathrm{C}-\mathrm{F}}=8.5 \mathrm{~Hz}\right), 113.84\left(J_{\mathrm{C}-\mathrm{F}}=23.5 \mathrm{~Hz}\right), 107.55\left(J_{\mathrm{C}-\mathrm{F}}=27 \mathrm{~Hz}\right), 66.23,48.48$. HRMS $(\mathrm{EI})$ calcd for $\mathrm{C}_{11} \mathrm{H}_{11} \mathrm{FN}_{2} \mathrm{OS}\left(\mathrm{M}^{+}\right)$238.0576, found 238.0573. 


\section{9f: 4-(6-Chlorobenzo[d]thiazol-2-yl)morpholine}

$\mathrm{N}$-(4-Chlorophenyl)morpholine-4-carbothioamide (0.05 g, $0.194 \mathrm{mmol})$, Oxone (0.119 g, 0.388 $\mathrm{mmol}), \mathrm{RuCl}_{3}(0.0018 \mathrm{~g}, 0.009 \mathrm{mmol}), \mathrm{DCE}(3 \mathrm{~mL})$. Product: brick red solid, yield: $0.036 \mathrm{~g}$ (73\%); m.p.: 142-144 ${ }^{\circ} \mathrm{C}$ [lit. $\left.{ }^{4} 144-145{ }^{\circ} \mathrm{C}\right] .{ }^{1} \mathrm{H} \mathrm{NMR}(\delta \mathrm{ppm}):\left(500 \mathrm{MHz}, \mathrm{CDCl}_{3}\right), 7.58(\mathrm{~d}, 1 \mathrm{H}$, aromatic C- $H, J=2 \mathrm{~Hz}), 7.46(\mathrm{~d}, 1 \mathrm{H}$, aromatic $\mathrm{C}-H, J=9 \mathrm{~Hz}), 7.26(\mathrm{dd}, 1 \mathrm{H}$, aromatic $\mathrm{C}-H, J=$ $2.5,9 \mathrm{~Hz}), 3.84\left(\mathrm{t}, 4 \mathrm{H}, \mathrm{sp}^{3} \mathrm{C}-H, J=5 \mathrm{~Hz}\right), 3.62\left(\mathrm{t}, 4 \mathrm{H}, \mathrm{sp}^{3} \mathrm{C}-H, J=5 \mathrm{~Hz}\right) .{ }^{13} \mathrm{C} \mathrm{NMR}(\delta \mathrm{ppm})$ : $\left(125 \mathrm{MHz}, \mathrm{CDCl}_{3}\right)$ : 169.06, 151.08, 131.77, 126.82, 126.60, 120.60, 119.96, 66.20, 48.49. HRMS (EI) calcd for $\mathrm{C}_{11} \mathrm{H}_{11} \mathrm{ClN}_{2} \mathrm{OS}\left(\mathrm{M}^{+}\right)$254.0281, found 254.0280.

\section{9g: 4-(6-Bromobenzo[d]thiazol-2-yl)morpholine}

$N$-(4-Bromophenyl)morpholine-4-carbothioamide (0.05 g, $0.167 \mathrm{mmol})$, Oxone (0.103 g, 0.334 $\mathrm{mmol}), \mathrm{RuCl}_{3}(0.0017 \mathrm{~g}, 0.008 \mathrm{mmol})$, DCE (3 mL). Product: brown solid, yield: $0.033 \mathrm{~g}(67 \%)$; m.p.: 165-166 ${ }^{\circ} \mathrm{C}\left[\right.$ lit. $\left.^{4} 165-167{ }^{\circ} \mathrm{C}\right] .{ }^{1} \mathrm{H} \mathrm{NMR}(\delta \mathrm{ppm}):\left(500 \mathrm{MHz}, \mathrm{CDCl}_{3}\right), 7.72(\mathrm{~d}, 1 \mathrm{H}$, aromatic $\mathrm{C}-H, J=1.35 \mathrm{~Hz}), 7.41(\mathrm{t}, 2 \mathrm{H}$, aromatic C- $H, J=1.73 \mathrm{~Hz}), 3.84\left(\mathrm{t}, 4 \mathrm{H}, \mathrm{sp}^{3} \mathrm{C}-H, J=4.9 \mathrm{~Hz}\right)$, $3.62\left(\mathrm{t}, 4 \mathrm{H}, \mathrm{sp}^{3} \mathrm{C}-H, J=4.9 \mathrm{~Hz}\right) .{ }^{13} \mathrm{C} \mathrm{NMR}(\delta \mathrm{ppm}):\left(125 \mathrm{MHz}, \mathrm{CDCl}_{3}\right): 169.06,151.49$, 132.22, 129.37, 123.29, 120.41, 114.02, 66.20, 48.47. HRMS (EI) calcd for $\mathrm{C}_{11} \mathrm{H}_{11} \mathrm{BrN}_{2} \mathrm{OS}\left(\mathrm{M}^{+}\right)$ 297.9775, found 297.9772.

\section{9h: Methyl 2-morpholinobenzo[d]thiazole-6-carboxylate}

Ethyl 4-(morpholine-4-carbothioamido)benzoate (0.05 g, $0.178 \mathrm{mmol})$, Oxone (0.109 g, 0.356 $\mathrm{mmol}), \mathrm{RuCl}_{3}(0.002 \mathrm{~g}, 0.009 \mathrm{mmol}), \mathrm{DCE}(3 \mathrm{~mL})$. Product: off-white solid, yield: $0.044 \mathrm{~g}$ (80\%); m.p.: $118-119{ }^{\circ} \mathrm{C} .{ }^{1} \mathrm{H}$ NMR $(\delta \mathrm{ppm}):\left(500 \mathrm{MHz}, \mathrm{CDCl}_{3}\right), 8.33(\mathrm{~d}, 1 \mathrm{H}$, aromatic $\mathrm{C}-H, J=$ $1.5 \mathrm{~Hz}), 8.02(\mathrm{dd}, 1 \mathrm{H}$, aromatic $\mathrm{C}-H, J=1.7,8.5 \mathrm{~Hz}), 7.55(\mathrm{~d}, 1 \mathrm{H}$, aromatic C- $H, J=8.5 \mathrm{~Hz})$, 4.38 (q, 2H, sp $\left.{ }^{3} \mathrm{C}-H, J=7.1 \mathrm{~Hz}\right), 3.85$ (t, $\left.4 \mathrm{H}, \mathrm{sp}^{3} \mathrm{C}-H, J=4.9 \mathrm{~Hz}\right), 3.68$ (t, 4H, sp ${ }^{3} \mathrm{C}-H, J=4.9$ $\mathrm{Hz}), 1.41\left(\mathrm{t}, 3 \mathrm{H}, \mathrm{sp}^{3} \mathrm{C}-H, J=7.1 \mathrm{~Hz}\right) .{ }^{13} \mathrm{C} \mathrm{NMR}(\delta \mathrm{ppm}):\left(125 \mathrm{MHz}, \mathrm{CDCl}_{3}\right): 171.01,166.46$, $156.28,130.44,128.01,123.67,122.81,118.52,66.21,60.88,48.49,14.42$. HRMS (EI) calcd for $\mathrm{C}_{13} \mathrm{H}_{14} \mathrm{~N}_{2} \mathrm{O}_{3} \mathrm{~S}\left(\mathrm{M}^{+}\right)$278.0725, found 278.0722.

\section{9i: 4-(5-Methoxybenzo[d]thiazol-2-yl)morpholine}

$\mathrm{N}$-(3-Methoxyphenyl)morpholine-4-carbothioamide (0.05 g, $0.198 \mathrm{mmol})$, Oxone (0.122 g, $0.396 \mathrm{mmol}), \mathrm{RuCl}_{3}(0.002 \mathrm{~g}, 0.01 \mathrm{mmol}), \mathrm{DCE}(3 \mathrm{~mL})$. Product: pale yellow solid, yield: 0.045 g (91\%); m.p.: $120-122{ }^{\circ} \mathrm{C}\left[\right.$ lit. $\left.^{3}{ }^{125-126}{ }^{\circ} \mathrm{C}\right] .{ }^{1} \mathrm{H}$ NMR $(\delta \mathrm{ppm}):\left(500 \mathrm{MHz}, \mathrm{CDCl}_{3}\right), 7.46(\mathrm{~d}, 1 \mathrm{H}$, aromatic C- $H, J=8.5 \mathrm{~Hz}), 7.14(\mathrm{~d}, 1 \mathrm{H}$, aromatic C- $H, J=2.5 \mathrm{~Hz}), 6.74(\mathrm{dd}, 1 \mathrm{H}$, aromatic C- $H, J$ $=2.5,9 \mathrm{~Hz}), 3.83\left(\mathrm{t}, 7 \mathrm{H}, \mathrm{sp}^{3} \mathrm{C}-H, J=5 \mathrm{~Hz}\right), 3.61\left(\mathrm{t}, 4 \mathrm{H}, \mathrm{sp}^{3} \mathrm{C}-H, J=4.7 \mathrm{~Hz}\right) .{ }^{13} \mathrm{C} \mathrm{NMR}(\delta$ ppm): (125 MHz, $\left.\mathrm{CDCl}_{3}\right)$ : 170.35, 159.07, 153.69, 121.99, 121.03, 110.40, 103.43, 66.24, 55.54, 48.42. HRMS (EI) calcd for $\mathrm{C}_{12} \mathrm{H}_{14} \mathrm{~N}_{2} \mathrm{O}_{2} \mathrm{~S}\left(\mathrm{M}^{+}\right)$250.0776, found 250.0780. 


\section{9j: 4-(5-Nitrobenzo[d]thiazol-2-yl)morpholine}

$\mathrm{N}$-(3-Nitrophenyl)morpholine-4-carbothioamide (0.05 g, $0.187 \mathrm{mmol})$, Oxone $(0.115 \mathrm{~g}, 0.374$ $\mathrm{mmol}), \mathrm{RuCl}_{3}(0.0018 \mathrm{~g}, 0.009 \mathrm{mmol}), \mathrm{DCE}(3 \mathrm{~mL})$. Product: yellowish orange solid, yield: 0.038 g (77\%); m.p.: $172-175{ }^{\circ} \mathrm{C}$ [lit. $\left.{ }^{4} 172-174{ }^{\circ} \mathrm{C}\right] .{ }^{1} \mathrm{H}$ NMR $(\delta \mathrm{ppm}):\left(500 \mathrm{MHz}, \mathrm{CDCl}_{3}\right), 8.36$ $(\mathrm{d}, 1 \mathrm{H}$, aromatic C- $H, J=2 \mathrm{~Hz}), 7.97(\mathrm{dd}, 1 \mathrm{H}$, aromatic $\mathrm{C}-H, J=2,8.5 \mathrm{~Hz}), 7.70(\mathrm{~d}, 1 \mathrm{H}$, aromatic C- $H, J=9 \mathrm{~Hz}), 3.86\left(\mathrm{t}, 4 \mathrm{H}, \mathrm{sp}^{3} \mathrm{C}-H, J=4.92 \mathrm{~Hz}\right), 3.68\left(\mathrm{t}, 4 \mathrm{H}, \mathrm{sp}^{3} \mathrm{C}-H, J=4.9 \mathrm{~Hz}\right)$. ${ }^{13} \mathrm{C}$ NMR $\left(\delta\right.$ ppm): $\left(125 \mathrm{MHz}, \mathrm{CDCl}_{3}\right): 170.28,152.98,147.00,137.82,120.78,116.37,114.05$, 66.17, 48.52. HRMS (EI) calcd for $\mathrm{C}_{11} \mathrm{H}_{11} \mathrm{~N}_{3} \mathrm{O}_{3} \mathrm{~S}\left(\mathrm{M}^{+}\right)$265.0521, found 265.0519.

\section{9k: 4-(7-Nitrobenzo[d]thiazol-2-yl)morpholine}

$\mathrm{N}$-(3-Nitrophenyl)morpholine-4-carbothioamide $(0.05 \mathrm{~g}, 0.187 \mathrm{mmol})$, Oxone $(0.115 \mathrm{~g}, 0.374$ $\mathrm{mmol}), \mathrm{RuCl}_{3}(0.0018 \mathrm{~g}, 0.009 \mathrm{mmol}), \mathrm{DCE}(3 \mathrm{~mL})$. Product: orange solid, yield: $0.038 \mathrm{~g}$ (77\%); m.p.: $178-180{ }^{\circ} \mathrm{C} .{ }^{1} \mathrm{H}$ NMR $(\delta \mathrm{ppm}):\left(500 \mathrm{MHz}, \mathrm{CDCl}_{3}\right), 8.06(\mathrm{~d}, 1 \mathrm{H}$, aromatic $\mathrm{C}-H, J=$ $8 \mathrm{~Hz}), 7.82(\mathrm{~d}, 1 \mathrm{H}$, aromatic $\mathrm{C}-H, J=7.5 \mathrm{~Hz}), 7.46(\mathrm{t}, 1 \mathrm{H}$, aromatic C- $H, J=8 \mathrm{~Hz}), 3.87(\mathrm{t}, 4 \mathrm{H}$, $\left.\mathrm{sp}^{3} \mathrm{C}-H, J=5 \mathrm{~Hz}\right), 3.72\left(\mathrm{t}, 4 \mathrm{H}, \mathrm{sp}^{3} \mathrm{C}-H, J=4.7 \mathrm{~Hz}\right) .{ }^{13} \mathrm{C} \mathrm{NMR}(\delta \mathrm{ppm}):\left(125 \mathrm{MHz}, \mathrm{CDCl}_{3}\right)$ : 171.27, 155.03, 142.16, 126.82, 126.21, 124.85, 117.33, 66.20, 48.33. HRMS (EI) calcd for $\mathrm{C}_{11} \mathrm{H}_{11} \mathrm{~N}_{3} \mathrm{O}_{3} \mathrm{~S}\left(\mathrm{M}^{+}\right)$265.0521, found 265.0517.

\section{A mixture of 91: 4-(5-chlorobenzo[d]thiazol-2-yl)morpholine and 9m: 4-(7- chlorobenzo[d]thiazol-2-yl)morpholine}

$\mathrm{N}$-(3-Chlorophenyl)morpholine-4-carbothioamide (0.05 g, $0.194 \mathrm{mmol})$, Oxone (0.119 g, 0.388 $\mathrm{mmol}), \mathrm{RuCl}_{3}(0.0018 \mathrm{~g}, 0.009 \mathrm{mmol})$, DCE ( $\left.3 \mathrm{~mL}\right)$. Product: creamish brown solid, yield: 0.034

g (69\%); m.p.: $112-113{ }^{\circ} \mathrm{C}\left[\right.$ lit. $\left.^{3} 112-113{ }^{\circ} \mathrm{C}\right] .{ }^{1} \mathrm{H}$ NMR $(\delta \mathrm{ppm}):\left(500 \mathrm{MHz}, \mathrm{CDCl}_{3}\right), 7.54(\mathrm{~d}$, $1 \mathrm{H}$, aromatic C- $H, J=2 \mathrm{~Hz}), 7.50(\mathrm{~d}, 1 \mathrm{H}$, aromatic C- $H, J=8.5 \mathrm{~Hz}), 7.45(\mathrm{dd}, 1 \mathrm{H}$, aromatic C$H, J=1,8 \mathrm{~Hz}), 7.25(\mathrm{t}, 1 \mathrm{H}$, aromatic C- $H, J=8 \mathrm{~Hz}), 7.10-7.06(\mathrm{~m}, 2 \mathrm{H}$, aromatic C- $H)$, 3.85$3.82\left(\mathrm{~m}, 8 \mathrm{H}, \mathrm{sp}^{3} \mathrm{C}-H\right), 3.65-3.61\left(\mathrm{~m}, 8 \mathrm{H}, \mathrm{sp}^{3} \mathrm{C}-H\right) .{ }^{13} \mathrm{C} \mathrm{NMR}(\delta \mathrm{ppm}):\left(125 \mathrm{MHz}, \mathrm{CDCl}_{3}\right)$ : $170.04,168.81,153.59,153.32,131.99,130.58,128.79,127.07,126.00,121.80,121.39,119.26$, 117.40, 66.20, 48.44. HRMS (EI) calcd for $\mathrm{C}_{11} \mathrm{H}_{11} \mathrm{ClN}_{2} \mathrm{OS}\left(\mathrm{M}^{+}\right)$254.0281, found 254.0284.

\section{9n: 4-(4-Chlorobenzo[d]thiazol-2-yl)morpholine}

$N$-(2-Chlorophenyl)morpholine-4-carbothioamide (0.05 g, $0.194 \mathrm{mmol})$, Oxone (0.119 g, 0.388 $\mathrm{mmol}), \mathrm{RuCl}_{3}(0.0018 \mathrm{~g}, 0.009 \mathrm{mmol})$, DCE $(3 \mathrm{~mL})$. Product: yellowish brown solid, yield: 0.032g (64\%); m.p.: 101-102 ${ }^{\circ} \mathrm{C}\left[\right.$ lit. $\left.^{3}{ }^{101-102}{ }^{\circ} \mathrm{C}\right] .{ }^{1} \mathrm{H}$ NMR $(\delta \mathrm{ppm}):\left(500 \mathrm{MHz}, \mathrm{CDCl}_{3}\right), 7.50$ $(\mathrm{dd}, 1 \mathrm{H}$, aromatic C- $H, J=1,8 \mathrm{~Hz}), 7.33(\mathrm{~d}, 1 \mathrm{H}$, aromatic C- $H, \mathrm{~J}=1,7.5 \mathrm{~Hz}), 7.02(\mathrm{t}, 1 \mathrm{H}$, aromatic C- $H, J=8 \mathrm{~Hz}), 3.85\left(\mathrm{t}, 4 \mathrm{H}, \mathrm{sp}^{3} \mathrm{C}-H, J=4.7 \mathrm{~Hz}\right), 3.67\left(\mathrm{t}, 4 \mathrm{H}, \mathrm{sp}^{3} \mathrm{C}-H, J=5 \mathrm{~Hz}\right) .{ }^{13} \mathrm{C}$ NMR $(\delta \mathrm{ppm}):\left(125 \mathrm{MHz}, \mathrm{CDCl}_{3}\right): 168.96,149.58,147.52,131.73,126.40,122.00,119.25$, 66.25, 48.45. HRMS (EI) calcd for $\mathrm{C}_{11} \mathrm{H}_{11} \mathrm{ClN}_{2} \mathrm{OS}\left(\mathrm{M}^{+}\right)$254.0281, found 254.0280. 


\section{9o:4-(4-Bromobenzo[d]thiazol-2-yl)morpholine}

$N$-(2-Bromophenyl)morpholine-4-carbothioamide (0.05 g, $0.166 \mathrm{mmol})$, Oxone $(0.102 \mathrm{~g}, 0.332$ $\mathrm{mmol}), \mathrm{RuCl}_{3}(0.0016 \mathrm{~g}, 0.008 \mathrm{mmol})$, DCE $(3 \mathrm{~mL})$. Product: red solid, yield: $0.039 \mathrm{~g}(78 \%)$; m.p.: 81-85 ${ }^{\circ} \mathrm{C} .{ }^{1} \mathrm{H}$ NMR $(\delta \mathrm{ppm}):\left(500 \mathrm{MHz}, \mathrm{CDCl}_{3}\right), 7.54(\mathrm{~d}, 1 \mathrm{H}$, aromatic $\mathrm{C}-H, J=7 \mathrm{~Hz})$, $7.50(\mathrm{~d}, 1 \mathrm{H}$, aromatic C- $H, J=7.5 \mathrm{~Hz}), 6.95(\mathrm{t}, 1 \mathrm{H}$, aromatic C- $H, J=8 \mathrm{~Hz}), 3.85\left(\mathrm{t}, 4 \mathrm{H}, \mathrm{sp}^{3} \mathrm{C}-\right.$ $H, J=5 \mathrm{~Hz}), 3.66\left(\mathrm{t}, 4 \mathrm{H}, \mathrm{sp}^{3} \mathrm{C}-H, J=5 \mathrm{~Hz}\right) .{ }^{13} \mathrm{C} \mathrm{NMR}(\delta \mathrm{ppm}):\left(125 \mathrm{MHz}, \mathrm{CDCl}_{3}\right): 168.61$, $150.82,131.10,129.47,122.39,119.90,112,43,66.26,48.41$. HRMS (EI) calcd for $\mathrm{C}_{11} \mathrm{H}_{11} \mathrm{BrN}_{2} \mathrm{OS}\left(\mathrm{M}^{+}\right)$297.9775, found 297.9779.

\section{9p: 4-(4-iodobenzo[d]thiazol-2-yl)morpholine}

$N$-(2-Iodophenyl)morpholine-4-carbothioamide (0.05 g, $0.143 \mathrm{mmol})$, Oxone (0.088 g, 0.286 $\mathrm{mmol}), \mathrm{RuCl}_{3}(0.0014 \mathrm{~g}, 0.007 \mathrm{mmol}), \mathrm{DCE}(3 \mathrm{~mL})$. Product: off-white oil, yield: $0.027 \mathrm{~g}$ $(55 \%) ;{ }^{1} \mathrm{H} \mathrm{NMR}(\delta \mathrm{ppm}):\left(500 \mathrm{MHz}, \mathrm{CDCl}_{3}\right), 7.72(\mathrm{dd}, 1 \mathrm{H}$, aromatic $\mathrm{C}-H, J=1,8 \mathrm{~Hz}), 7.56$ $(\mathrm{dd}, 1 \mathrm{H}$, aromatic C- $H, J=1,7.5 \mathrm{~Hz}), 6.81(\mathrm{t}, 1 \mathrm{H}$, aromatic C- $H, J=7.7 \mathrm{~Hz}), 3.85\left(\mathrm{t}, 4 \mathrm{H}, \mathrm{sp}^{3} \mathrm{C}-\right.$ $H, J=5 \mathrm{~Hz}), 3.66\left(\mathrm{t}, 4 \mathrm{H}, \mathrm{sp}^{3} \mathrm{C}-H, J=5 \mathrm{~Hz}\right) .{ }^{13} \mathrm{C} \mathrm{NMR}(\delta \mathrm{ppm}):\left(125 \mathrm{MHz}, \mathrm{CDCl}_{3}\right): 167.72$, 153.53, 135.59, 129.01, 122.92, 120.78, 85.85, 66.26, 48.39. HRMS (EI) calcd for $\mathrm{C}_{11} \mathrm{H}_{11} \mathrm{IN}_{2} \mathrm{OS}$ $\left(\mathrm{M}^{+}\right)$345.9637, found 345.9639 .

\section{9q: $N$-Phenylbenzo[d]thiazol-2-amine}

1,3-Diphenylthiourea $(0.05 \mathrm{~g}, 0.219 \mathrm{mmol})$, Oxone $(0.135 \mathrm{~g}, 0.438 \mathrm{mmol}), \mathrm{RuCl}_{3}(0.0021 \mathrm{~g}$, $0.010 \mathrm{mmol})$, DCE (3 mL). Product: cream solid, yield: $0.041 \mathrm{~g}(82 \%)$; m.p.: $158-159^{\circ} \mathrm{C}$ [lit. $^{5}$ 157-159 $\left.{ }^{\circ} \mathrm{C}\right] .{ }^{1} \mathrm{H}$ NMR $(\delta \mathrm{ppm}):\left(500 \mathrm{MHz} \mathrm{CDCl}_{3}\right), 8.82(\mathrm{bs}, 1 \mathrm{H}, \mathrm{N}-H), 7.64(\mathrm{dd}, 1 \mathrm{H}$, aromatic $\mathrm{C}-H, J=0.5,7.5 \mathrm{~Hz}), 7.59(\mathrm{~d}, 2 \mathrm{H}$, aromatic C- $H, J=8 \mathrm{~Hz}), 7.52(\mathrm{dd}, 2 \mathrm{H}$, aromatic $\mathrm{C}-H, J=1$, $8.5 \mathrm{~Hz}), 7.42(\mathrm{t}, 2 \mathrm{H}$, aromatic C- $H, J=8 \mathrm{~Hz}), 7.34(\mathrm{dt}, 1 \mathrm{H}$, aromatic C- $H, J=1,8 \mathrm{~Hz}), 7.17(\mathrm{~m}$, $2 \mathrm{H}$, aromatic $\mathrm{C}-H) .{ }^{13} \mathrm{C}$ NMR $(\delta \mathrm{ppm}):\left(125 \mathrm{MHz}, \mathrm{CDCl}_{3}\right): 164.71,151.43,139.87,129.92$, 129.61, 126.18, 124.41, 122.44, 120.89, 120.28, 119.40. HRMS (EI) calcd for $\mathrm{C}_{13} \mathrm{H}_{10} \mathrm{~N}_{2} \mathrm{~S}\left(\mathrm{M}^{+}\right)$ 226.0565 , found 226.0560 .

\section{9r: $N$-(4-Chloro-3-fluorophenyl)-5-methoxybenzo[d]thiazol-2-amine}

1-(4-Chloro-3-fluorophenyl)-3-(4-methoxyphenyl)thiourea (0.05 g, $0.160 \mathrm{mmol})$, Oxone (0.098 g, $0.320 \mathrm{mmol}), \mathrm{RuCl}_{3}(0.0016 \mathrm{~g}, 0.008 \mathrm{mmol})$, DCE $(3 \mathrm{~mL})$. Product: off white solid, yield: 0.038 g (76\%); m.p.: 186-189 ${ }^{\circ} \mathrm{C} .{ }^{1} \mathrm{H}$ NMR $\left(\delta\right.$ ppm): $\left(500 \mathrm{MHz}, \mathrm{CDCl}_{3}+30 \mu \mathrm{L}\right.$ of DMSO- $\left.d_{6}\right)$, 9.72 (brs, $1 \mathrm{H}, \mathrm{N}-\mathrm{H}), 7.89-7.87(\mathrm{~m}, 1 \mathrm{H}$, aromatic $\mathrm{C}-H), 7.47-7.45(\mathrm{~m}, 1 \mathrm{H}$, aromatic $\mathrm{C}-H), 7.34(\mathrm{~d}$, $1 \mathrm{H}$, aromatic C- $H, J=8.6 \mathrm{~Hz}), 7.10(\mathrm{~d}, 1 \mathrm{H}$, aromatic C- $H, J=2.4 \mathrm{~Hz}), 6.96(\mathrm{t}, 1 \mathrm{H}$, aromatic C$H, J=8.8 \mathrm{~Hz}), 6.66-6.64(\mathrm{~m}, 1 \mathrm{H}$, aromatic $\mathrm{C}-H, J=2.5,8.6 \mathrm{~Hz}), 3.73\left(\mathrm{~s}, 3 \mathrm{H}, \mathrm{OCH}_{3}\right) .{ }^{13} \mathrm{C} \mathrm{NMR}$ $(\delta \mathrm{ppm}):\left(125 \mathrm{MHz}, \mathrm{CDCl}_{3}+30 \mu \mathrm{L} \mathrm{DMSO}-d_{6}\right):\left(125 \mathrm{MHz}, \mathrm{CDCl}_{3}\right): 163.10,158.77,154.19$, $153.34,152.25,137.60,121.65,120.69,119.93,117.85,117.80,116.49,116.32$, 111.02, 103.97, 55.49 . 


\section{9s: 2-(4-(2-Fluorophenyl)piperazin-1-yl)benzo[d]thiazole}

4-(2-Fluorophenyl)-N-phenylpiperidine-1-carbothioamide (0.05 g, $0.158 \mathrm{mmol})$, Oxone (0.097 $\mathrm{g}, 0.317 \mathrm{mmol}), \mathrm{RuCl}_{3}(0.0016 \mathrm{~g}, 0.007 \mathrm{mmol})$, DCE $(3 \mathrm{~mL})$. Product: brown solid, yield: 0.037 g (74\%); m.p.: 130-132 ${ }^{\circ} \mathrm{C}\left[\right.$ lit. $\left.^{6} 106-107{ }^{\circ} \mathrm{C}\right] .{ }^{1} \mathrm{H}$ NMR $(\delta \mathrm{ppm}):\left(500 \mathrm{MHz}, \mathrm{CDCl}_{3}\right), 7.63(\mathrm{~d}, 1 \mathrm{H}$, aromatic C- $H, J=8 \mathrm{~Hz}), 7.59(\mathrm{~d}, 1 \mathrm{H}$, aromatic C- $H, J=8 \mathrm{~Hz}), 7.32(\mathrm{dt}, 1 \mathrm{H}$, aromatic $\mathrm{C}-H, J=$ $1,8 \mathrm{~Hz}), 7.12-7.06(\mathrm{~m}, 3 \mathrm{H}$, aromatic $\mathrm{C}-H), 7.02-6.97(\mathrm{~m}, 2 \mathrm{H}$, aromatic $\mathrm{C}-H), 3.83\left(\mathrm{t}, 4 \mathrm{H}, \mathrm{sp}^{3} \mathrm{C}-\right.$ $H, J=5 \mathrm{~Hz}), 3.23\left(\mathrm{t}, 4 \mathrm{H}, \mathrm{sp}^{3} \mathrm{C}-H, J=5 \mathrm{~Hz}\right) .{ }^{13} \mathrm{C} \mathrm{NMR}(\delta \mathrm{ppm}):\left(125 \mathrm{MHz}, \mathrm{CDCl}_{3}\right):^{`} 168.78$, $154.84\left(J_{\mathrm{C}-\mathrm{F}}=258.75 \mathrm{~Hz}\right), 139.64\left(J_{\mathrm{C}-\mathrm{F}}=7.4 \mathrm{~Hz}\right), 130.76,126.10,124.60\left(J_{\mathrm{C}-\mathrm{F}}=2.5 \mathrm{~Hz}\right), 123.27$ $\left(J_{\mathrm{C}-\mathrm{F}}=7.5 \mathrm{~Hz}\right), 121.61,120.77,119.28\left(J_{\mathrm{C}-\mathrm{F}}=5 \mathrm{~Hz}\right), 116.32\left(J_{\mathrm{C}-\mathrm{F}}=20 \mathrm{~Hz}\right), 50.13\left(J_{\mathrm{C}-\mathrm{F}}=2.5 \mathrm{~Hz}\right)$, 48.56. HRMS (EI) calcd for $\mathrm{C}_{17} \mathrm{H}_{16} \mathrm{FN}_{3} \mathrm{~S}\left(\mathrm{M}^{+}\right)$313.1049, found 313.1039 .

\section{9t: 2-(4-(4-Fluorophenyl)piperazin-1-yl)benzo[d]thiazole}

4-(4-Fluorophenyl)-N-phenylpiperidine-1-carbothioamide (0.05 g, $0.158 \mathrm{mmol})$, Oxone (0.097 $\mathrm{g}, 0.317 \mathrm{mmol}), \mathrm{RuCl}_{3}(0.0016 \mathrm{~g}, 0.007 \mathrm{mmol})$, DCE $(3 \mathrm{~mL})$. Product: light pink solid, yield: 0.039 g (79\%); m.p.: 98-100 ${ }^{\circ} \mathrm{C} .{ }^{1} \mathrm{H}$ NMR $\left(\delta\right.$ ppm): $\left(500 \mathrm{MHz}, \mathrm{CDCl}_{3}\right), 7.61$ (d, 1H, aromatic C$H, J=7.5 \mathrm{~Hz}), 7.32$ (d, $1 \mathrm{H}$, aromatic C- $H, J=8 \mathrm{~Hz}), 7.03-6.99$ (m, 2H, aromatic C- $H$ ), 6.96$6.92(\mathrm{~m}, 3 \mathrm{H}$, aromatic C- $H), 3.83\left(\mathrm{t}, 4 \mathrm{H}, \mathrm{sp}^{3} \mathrm{C}-H, J=5 \mathrm{~Hz}\right), 3.23\left(\mathrm{t}, 4 \mathrm{H}, \mathrm{sp}^{3} \mathrm{C}-H, J=5.3 \mathrm{~Hz}\right)$. ${ }^{13} \mathrm{C}$ NMR $(\delta \mathrm{ppm}):\left(125 \mathrm{MHz}, \mathrm{CDCl}_{3}\right):{ }^{\prime} 168.28,157.79\left(J_{\mathrm{C}-\mathrm{F}}=237.5 \mathrm{~Hz}\right), 150.95,147.54$, 131.33, 129.48, 122.37, 119.94, $118.94\left(J_{\mathrm{C}-\mathrm{F}}=7.5 \mathrm{~Hz}\right), 115.82\left(J_{\mathrm{C}-\mathrm{F}}=22.5 \mathrm{~Hz}\right), 112.35,50.21$, 48.31. HRMS (EI) calcd for $\mathrm{C}_{17} \mathrm{H}_{16} \mathrm{FN}_{3} \mathrm{~S}\left(\mathrm{M}^{+}\right)$313.1049, found 313.1043 .

\section{9u: 5-Methoxy-N-phenylbenzo[d]thiazol-2-amine}

1-(3-Methoxyphenyl)-3-phenylthiourea (0.05 g, $0.193 \mathrm{mmol})$, Oxone (0.119 g, $0.386 \mathrm{mmol})$, $\mathrm{RuCl}_{3}$ (0.002 g, $\left.0.010 \mathrm{mmol}\right)$, DCE (3 mL). Product: off white solid, yield: $0.045 \mathrm{~g}(90 \%)$; m.p.: 177-178.3 ${ }^{\circ} \mathrm{C} .{ }^{1} \mathrm{H}$ NMR $(\delta \mathrm{ppm}):\left(500 \mathrm{MHz}, \mathrm{CDCl}_{3}\right), 7.76(\mathrm{dd}, 3 \mathrm{H}$, aromatic $\mathrm{C}-H, J=7.5,8.5$ $\mathrm{Hz})$, 7.43-7.40 (m, 2H, aromatic C- $H, J=6.5 \mathrm{~Hz}), 7.18-7.14(\mathrm{~m}, 2 \mathrm{H}$, aromatic C- $H), 6.81$ (dd, $1 \mathrm{H}$, aromatic $\mathrm{C}-H, J=2.5,8.5 \mathrm{~Hz}), 3.82\left(\mathrm{~s}, 3 \mathrm{H}, \mathrm{sp}^{3} \mathrm{C}-H, J=4.7 \mathrm{~Hz}\right) .{ }^{13} \mathrm{C} \mathrm{NMR}(\delta \mathrm{ppm}):(125$ $\left.\mathrm{MHz}, \mathrm{CDCl}_{3}\right): 166.04,159.08,152.62,139.87,129.56,124.39,121.33,121.10,120.35,111.17$, 103.46, 55.52. HRMS (EI) calcd for $\mathrm{C}_{14} \mathrm{H}_{12} \mathrm{~N}_{2} \mathrm{OS}\left(\mathrm{M}^{+}\right)$256.0670, found 256.0675.

\section{9v: 5-Methoxy-N-(3-nitrophenyl)benzo[d]thiazol-2-amine}

1-(3-Methoxyphenyl)-3-(3-nitrophenyl)thiourea (0.05 g, $0.164 \mathrm{mmol})$, Oxone (0.101 g, 0.328 $\mathrm{mmol}), \mathrm{RuCl}_{3}(0.0017 \mathrm{~g}, 0.008 \mathrm{mmol})$, DCE $(3 \mathrm{~mL})$. Product: yellow solid, yield: $0.037 \mathrm{~g}$ (74 \%); m.p.: $156-159{ }^{\circ} \mathrm{C} .{ }^{1} \mathrm{H} \mathrm{NMR}(\delta \mathrm{ppm}):\left(500 \mathrm{MHz}, \mathrm{CDCl}_{3}\right), 8.54(\mathrm{t}, 1 \mathrm{H}$, aromatic $\mathrm{C}-H, J=1.7$ $\mathrm{Hz}), 7.94(\mathrm{dd}, 1 \mathrm{H}$, aromatic C- $H, J=2,8 \mathrm{~Hz}), 7.89$ (dd, $1 \mathrm{H}$, aromatic C- $H, J=2,8.5 \mathrm{~Hz}), 7.56-$ $7.51(\mathrm{~m}, 3 \mathrm{H}$, aromatic C- $H), 7.25(\mathrm{~d}, 1 \mathrm{H}$, aromatic C- $H, J=2 \mathrm{~Hz}), 6.87(\mathrm{dd}, 1 \mathrm{H}$, aromatic C- $H, J$ $=2,8.5 \mathrm{~Hz}), 3.82\left(\mathrm{~s}, 3 \mathrm{H}, \mathrm{sp}^{3} \mathrm{C}-H, J=4.7 \mathrm{~Hz}\right) \cdot{ }^{13} \mathrm{C} \mathrm{NMR}(\delta \mathrm{ppm}):\left(125 \mathrm{MHz}, \mathrm{CDCl}_{3}\right): 163.25$, 159.30, 152.22, 148.97, 141.08, 139.33, 130.18, 124.33, 121.27, 117.86, 113.30, 112.32, 104.08, 55.64. HRMS (EI) calcd for $\mathrm{C}_{14} \mathrm{H}_{11} \mathrm{~N}_{3} \mathrm{O}_{3} \mathrm{~S}\left(\mathrm{M}^{+}\right)$303.0521, found 303.0525. 
Synthesis of 2-(2,3-diphenyl-1H-indol-1-yl)benzo[d]thiazole (16): A schlenk tube equipped with a stir-bar was charged with $\mathbf{9 q}(0.05 \mathrm{~g}, 0.22 \mathrm{mmol})$, diphenylacetylene $(0.039 \mathrm{~g}, 0.22$ $\mathrm{mmol})$. Then $3 \mathrm{ml}$ of DCE was added to the reaction tube via syringe. The reaction tube was purged with argon. Then after 5-10 min, $\left[\mathrm{Ru}(\mathrm{p} \text {-cymene }) \mathrm{Cl}_{2}\right]_{2}(0.007 \mathrm{~g}, 0.01 \mathrm{mmol}), \mathrm{AgSbF}_{6}$ $(0.076 \mathrm{~g}, 0.22 \mathrm{mmol}), \mathrm{Cu}(\mathrm{OAc})_{2}(0.04 \mathrm{~g}, 0.22 \mathrm{mmol})$ were added to the reaction mixture The mixture was stirred at $120{ }^{\circ} \mathrm{C}$ for $12 \mathrm{hrs}$. On the completion of the reaction on TLC, the reaction mixture was passed through Celite bed and concentrated under reduced pressure and then purified by silica gel chromatography (EtOAc: hexane) to furnish the desired product $\mathbf{1 6}$ as light yellow solid, $0.075 \mathrm{~g}$; $(84 \%)$; m.p.: $98-102{ }^{\circ} \mathrm{C} .{ }^{1} \mathrm{H}$ NMR $(\delta \mathrm{ppm}):\left(500 \mathrm{MHz}, \mathrm{CDCl}_{3}\right), 8.43(\mathrm{~d}$, $1 \mathrm{H}$, aromatic C- $H, J=8.5 \mathrm{~Hz}), 8.07(\mathrm{~d}, 1 \mathrm{H}$, aromatic C- $H, J=8 \mathrm{~Hz}), 7.74(\mathrm{~d}, 1 \mathrm{H}$, aromatic C- $H$, $J=7.5 \mathrm{~Hz}), 7.66(\mathrm{~d}, 1 \mathrm{H}$, aromatic C- $H, J=7.5 \mathrm{~Hz}), 7.50(\mathrm{dt}, 1 \mathrm{H}$, aromatic C- $H, J=1.5,8.5$ $\mathrm{Hz}), 7.43(\mathrm{dt}, 1 \mathrm{H}$, aromatic $\mathrm{C}-H, J=1,8.5 \mathrm{~Hz}), 7.38-7.31(\mathrm{~m}, 12 \mathrm{H}$, aromatic $\mathrm{C}-H) .{ }^{13} \mathrm{C}$ NMR $(\delta$ ppm): (125 MHz, $\left.\mathrm{CDCl}_{3}\right)$ : 158.78, 149.94, 137.57, 135.72, 134.50, 133.56, 131.90, 130.41, $130.10,128.85,128.78,128.50,128.36,126.72,126.27,124.76,124.64,123.01,122.78,121.14$, $120.86,119.79,113.63$. 


\section{Analytical Data of the compounds}

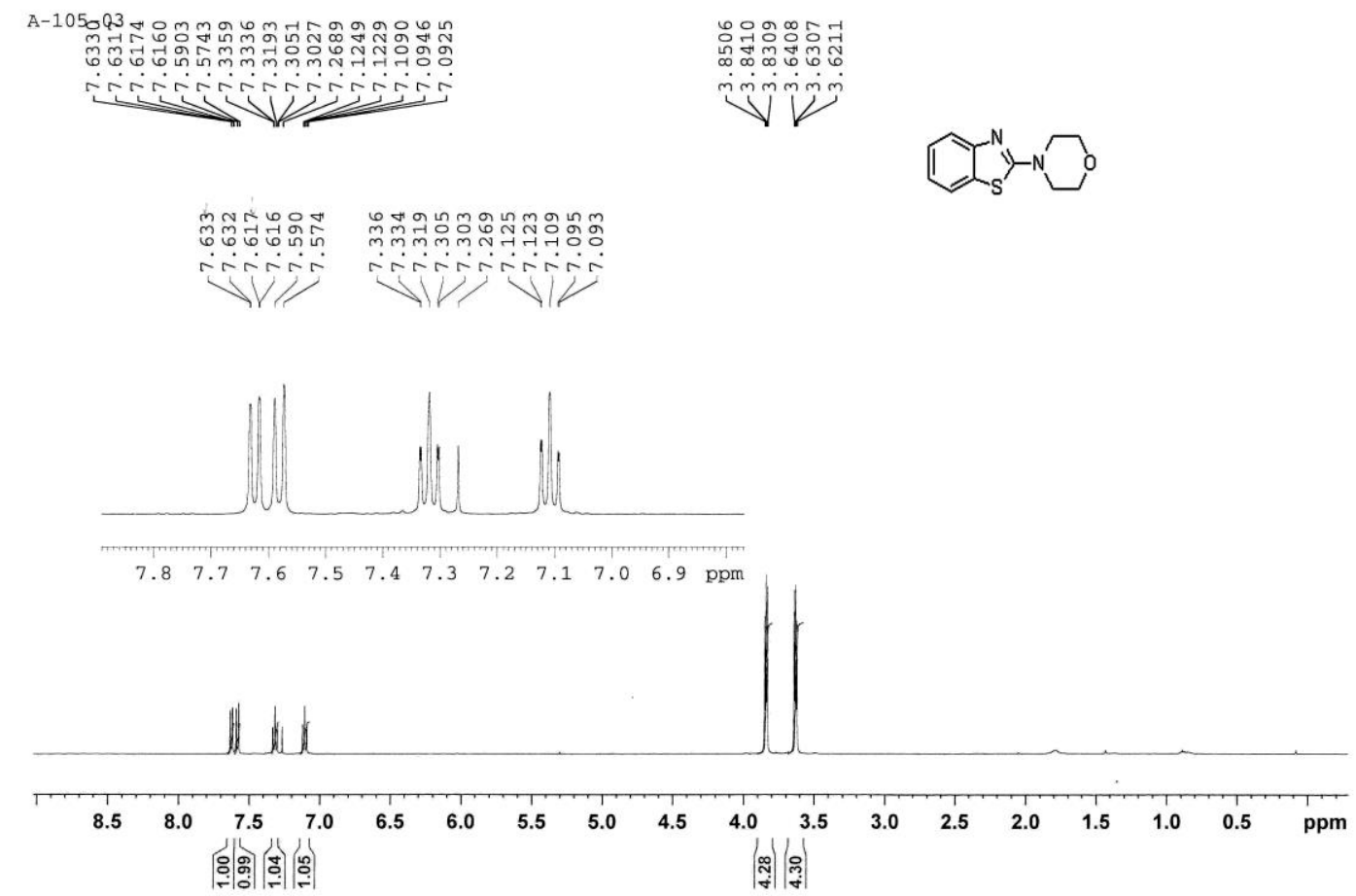

Figure S1: ${ }^{1} \mathrm{H}$ NMR spectra of 4-(benzo[d]thiazol-2-yl)morpholine (9a).

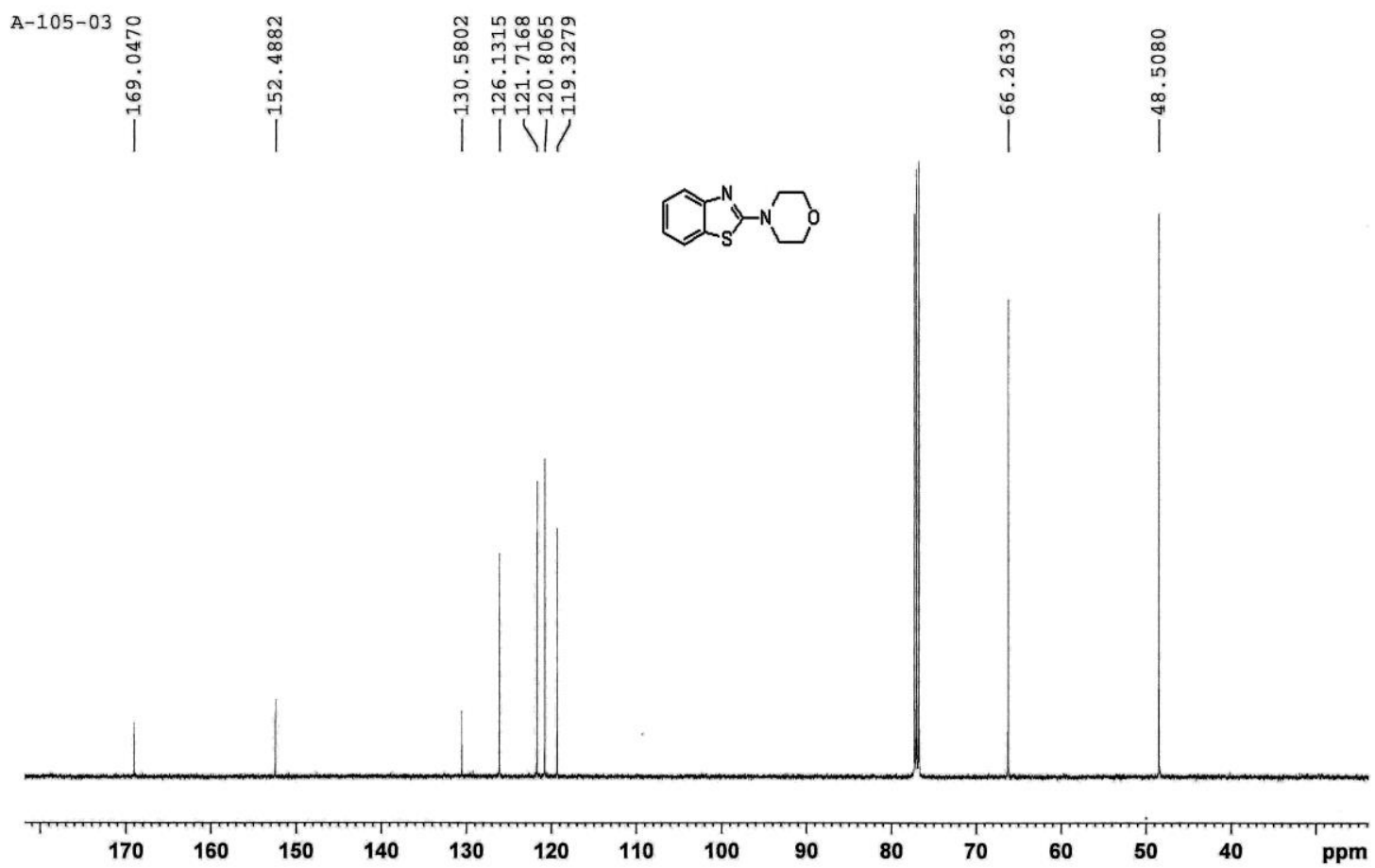

Figure S2: ${ }^{13} \mathrm{C}$ NMR spectra of4-(benzo[d]thiazol-2-yl)morpholine (9a). 


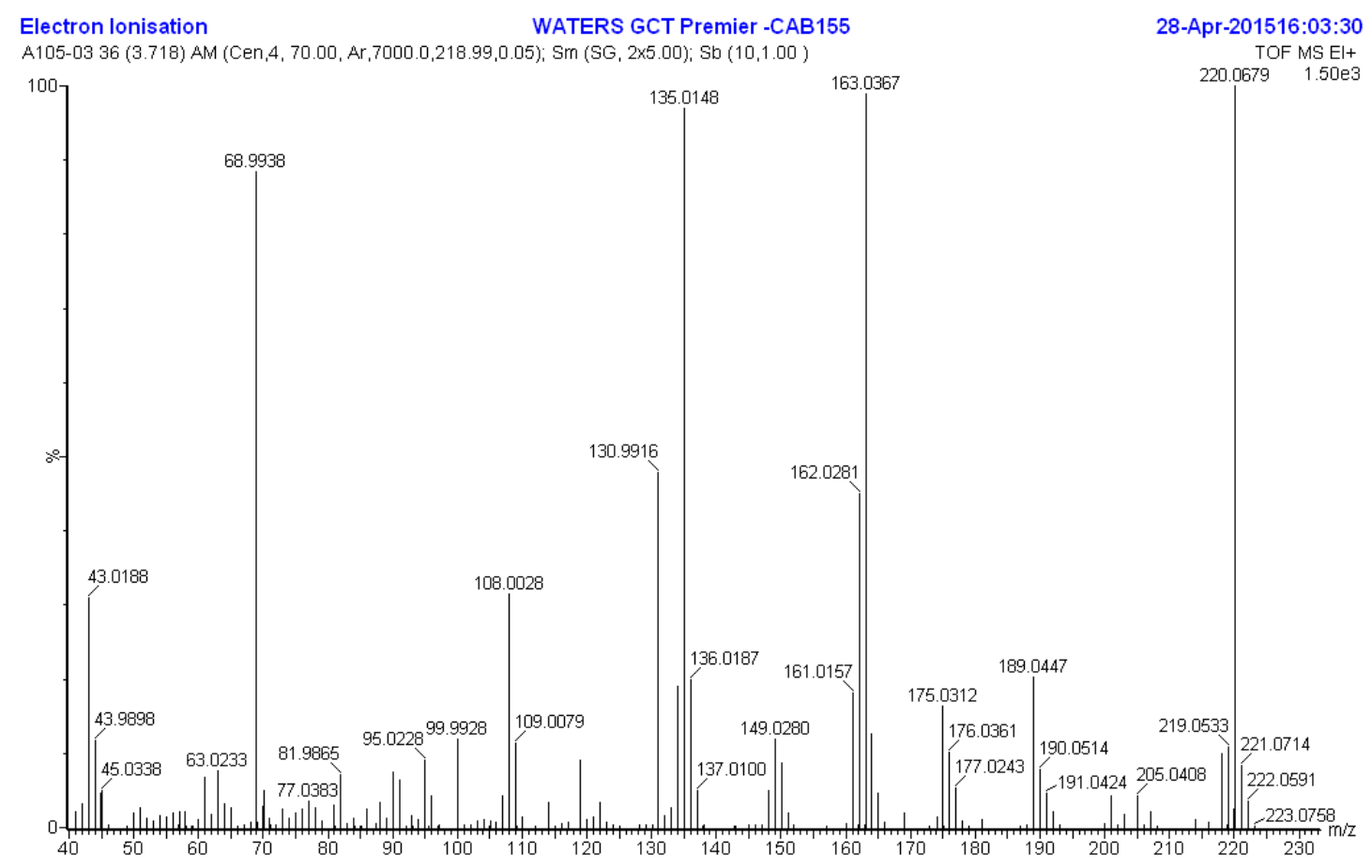

Figure S3: HRMS-EI spectra of 4-(benzo[d]thiazol-2-yl)morpholine (9a).

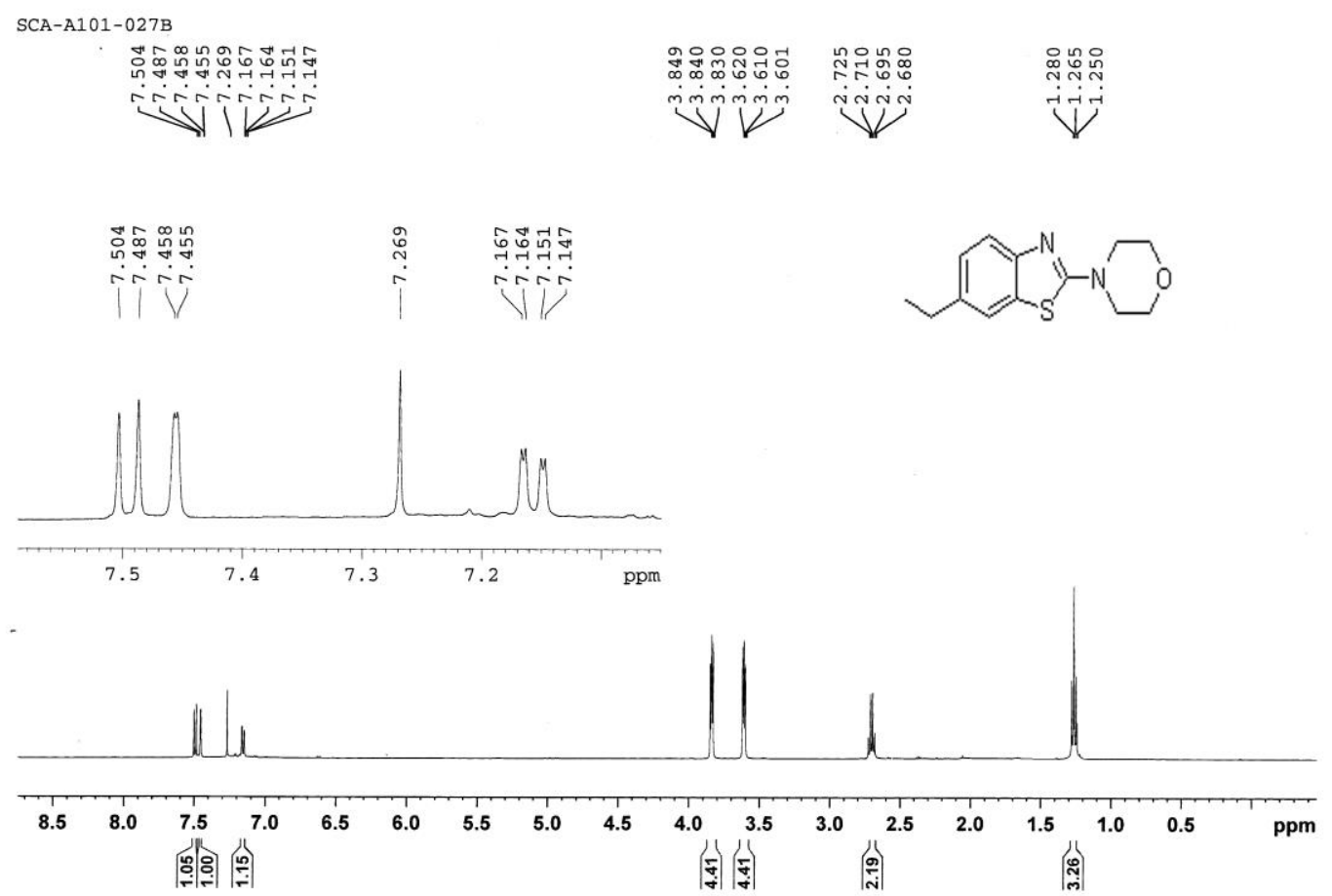

Figure S4: ${ }^{1} \mathrm{H}$ NMR spectra of 4-(6-ethylbenzo[d]thiazol-2-yl)morpholine (9b). 


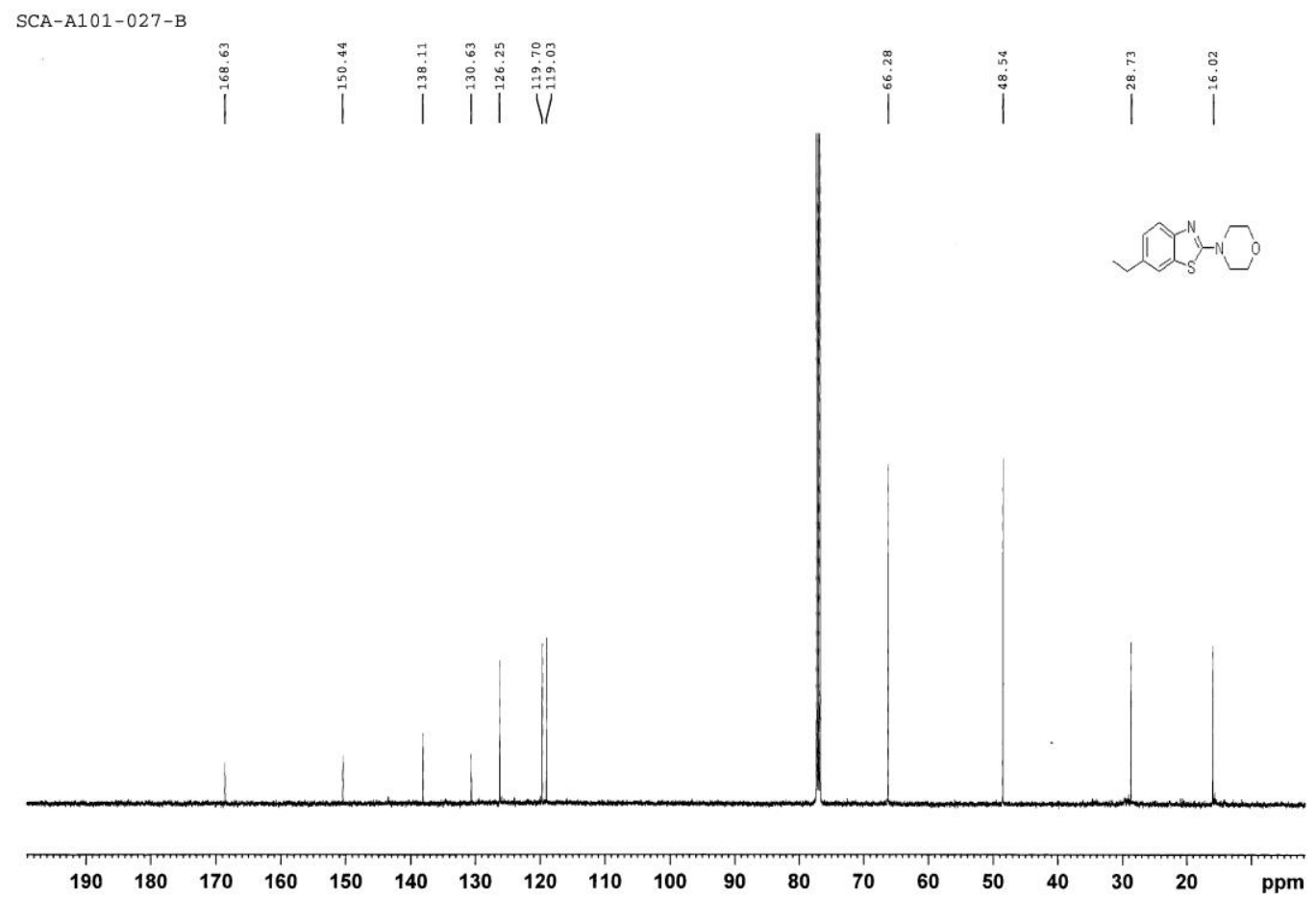

Figure S5: ${ }^{13} \mathrm{C}$ NMR spectra of 4-(6-ethylbenzo[d]thiazol-2-yl)morpholine (9b).

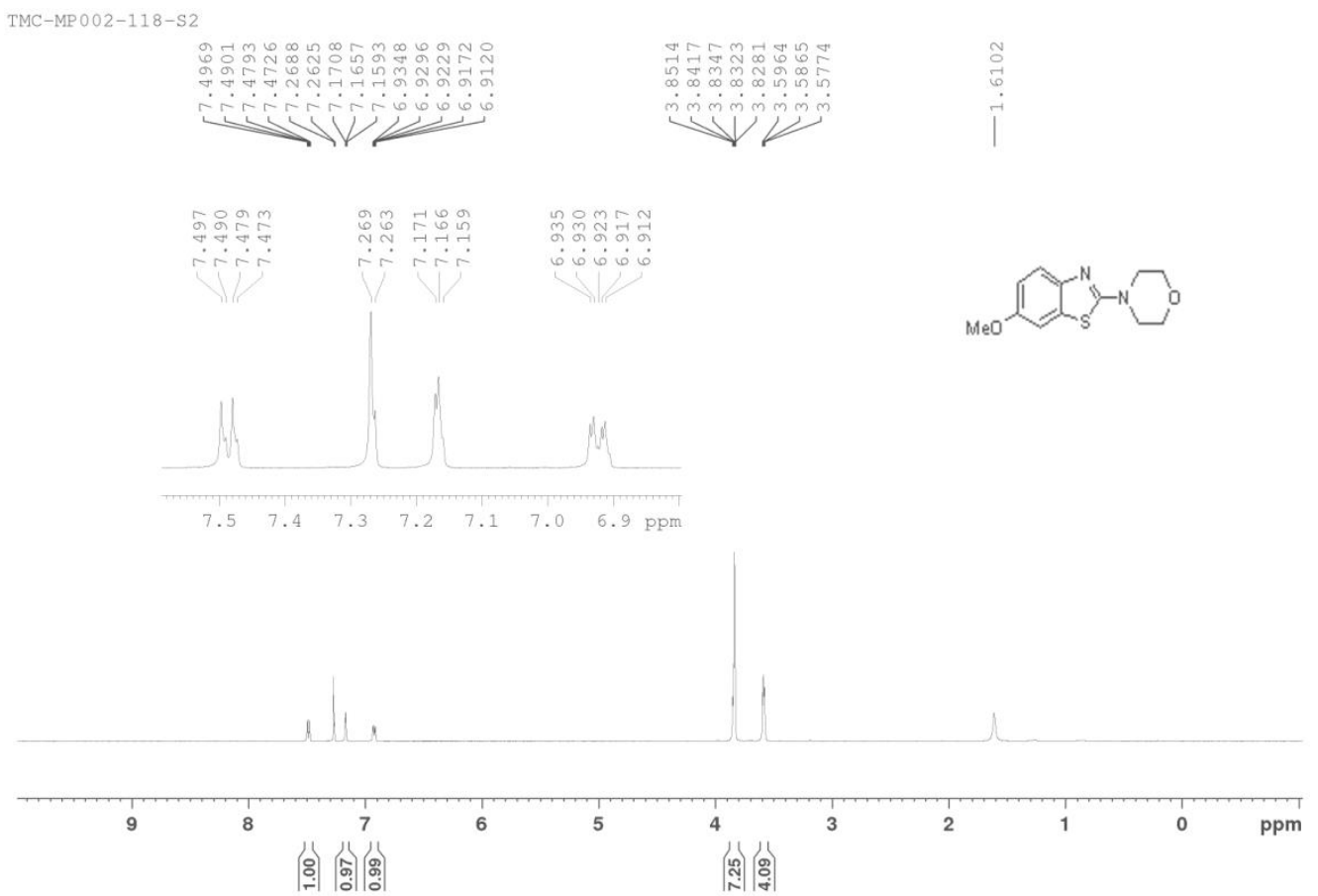

Figure S6: ${ }^{1}$ H NMR spectra of4-(6-methoxybenzo[d]thiazol-2-yl)morpholine (9c). 


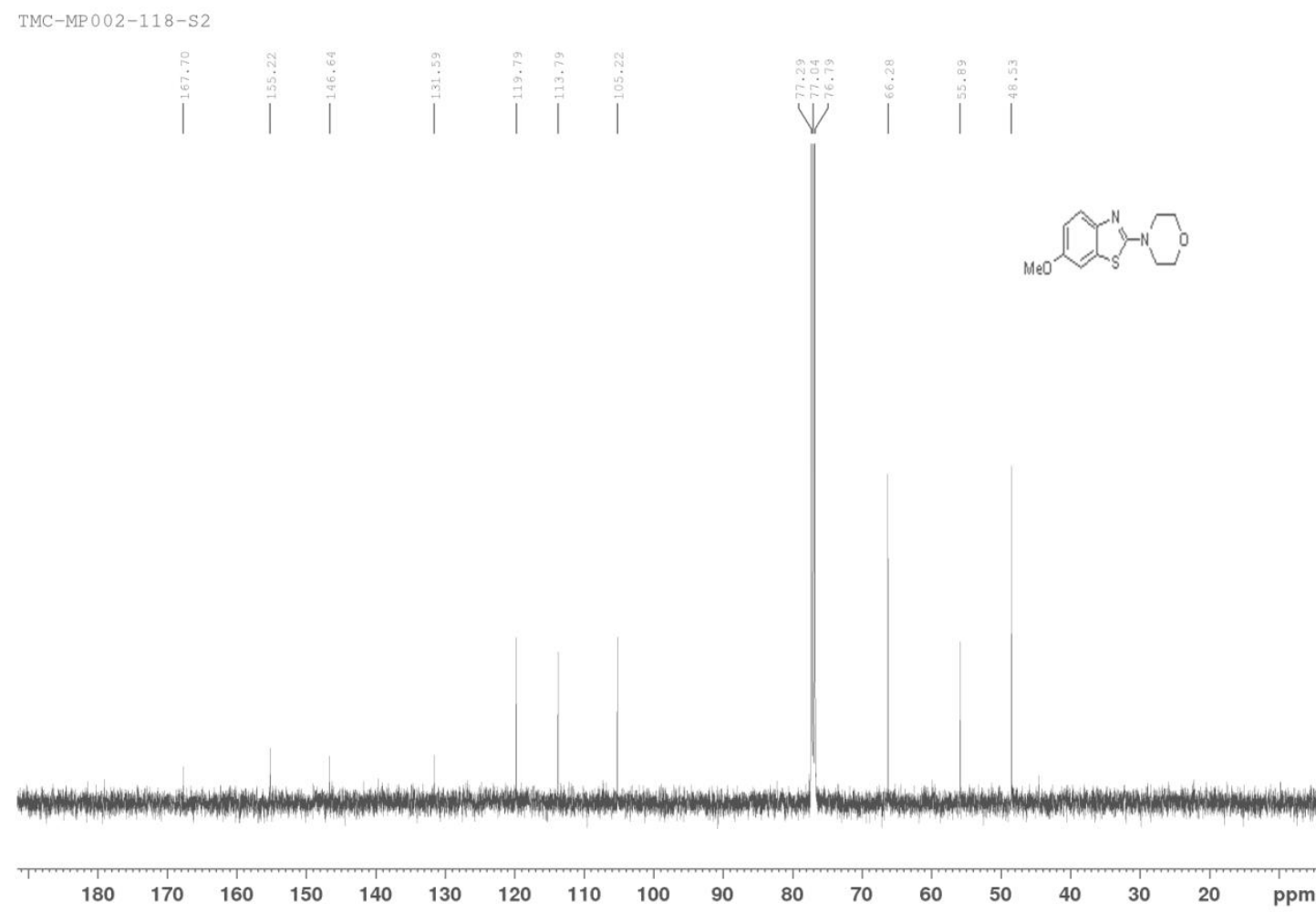

Figure S7: ${ }^{13} \mathrm{C}$ NMR spectra of 4-(6-methoxybenzo[d]thiazol-2-yl)morpholine (9c).

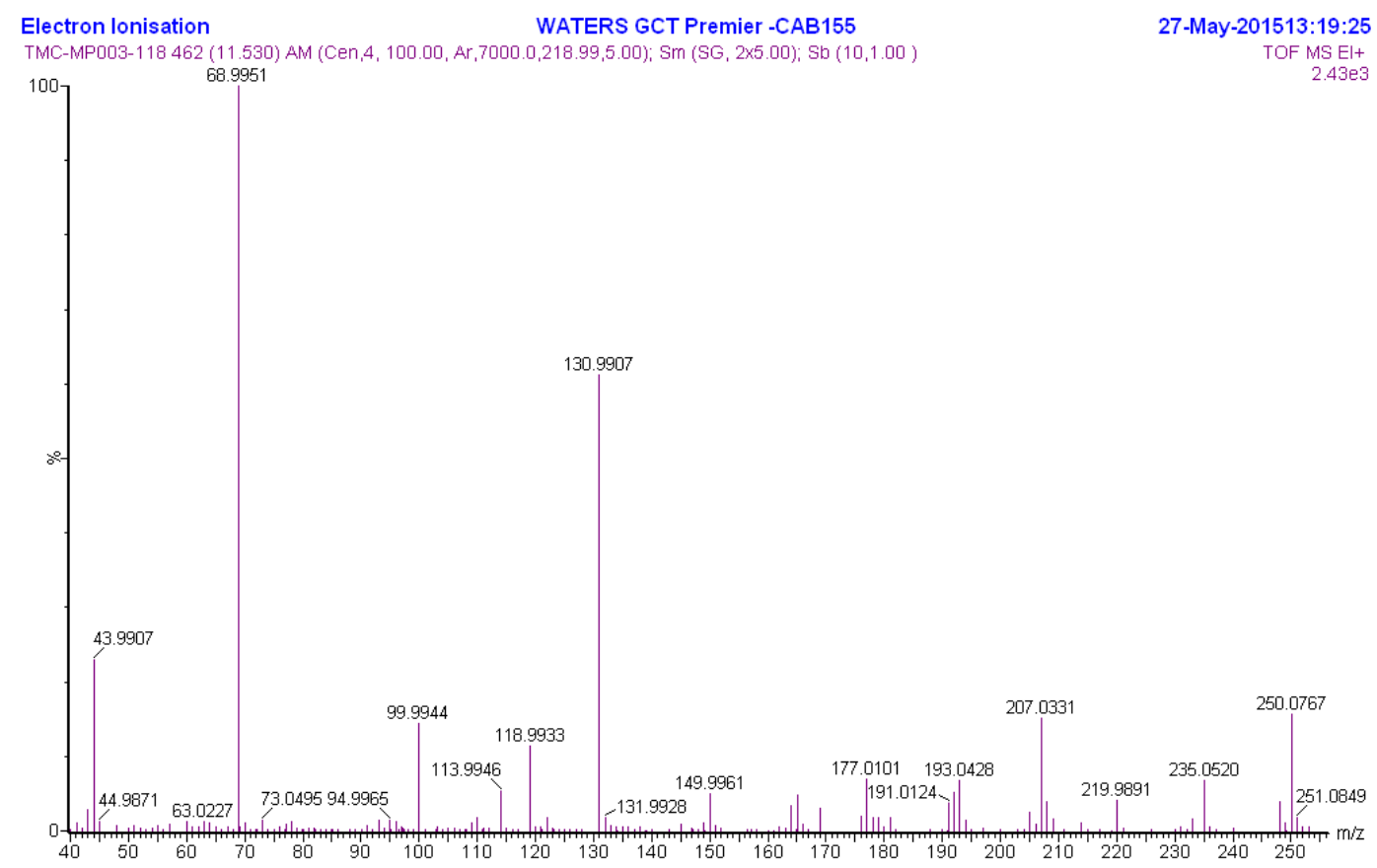

Figure S8: HRMS-EI spectra of 4-(6-methoxybenzo[d]thiazol-2-yl)morpholine (9c). 


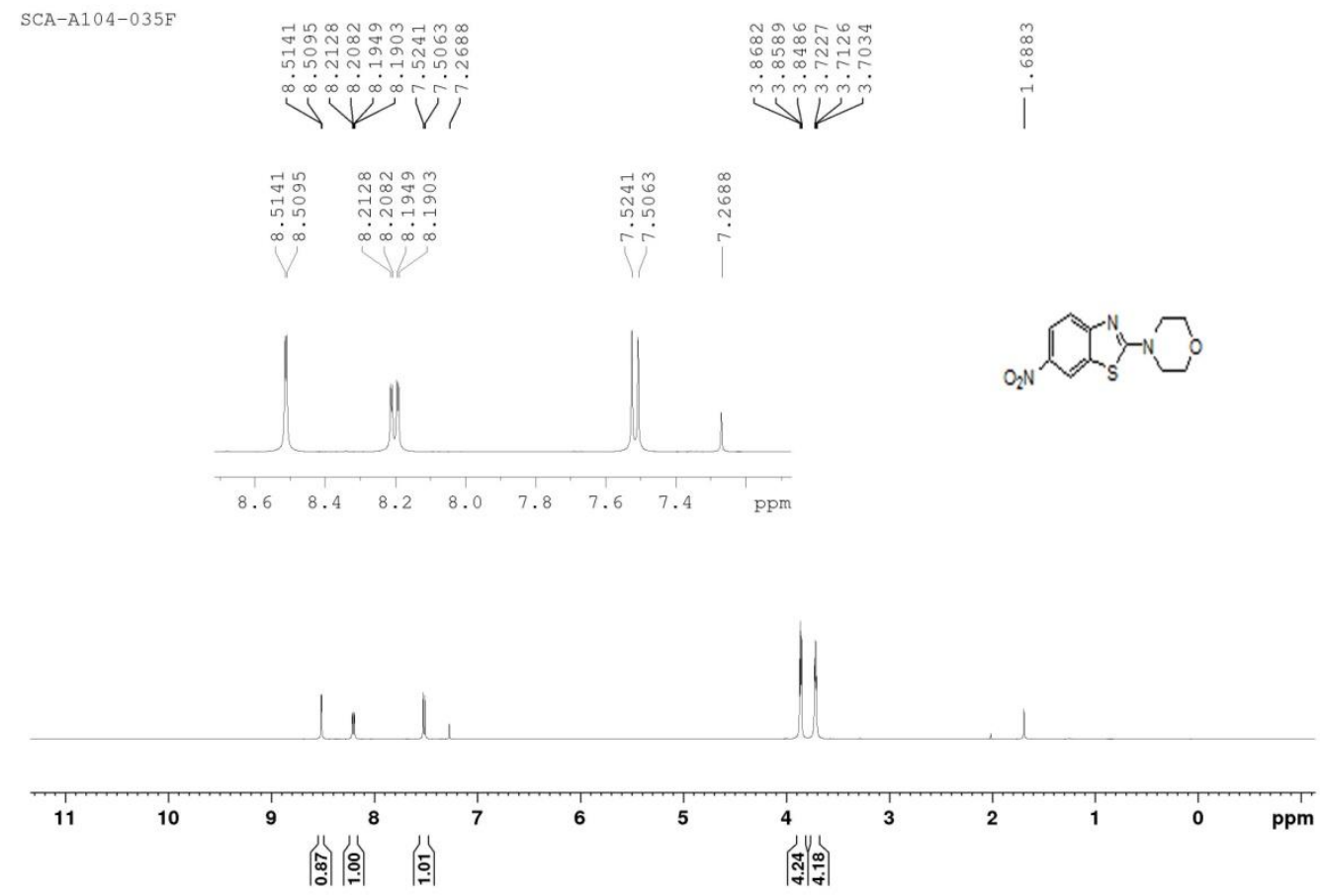

Figure S9: ${ }^{1} \mathrm{H}$ NMR spectra of4-(6-nitrobenzo[d]thiazol-2-yl)morpholine (9d).

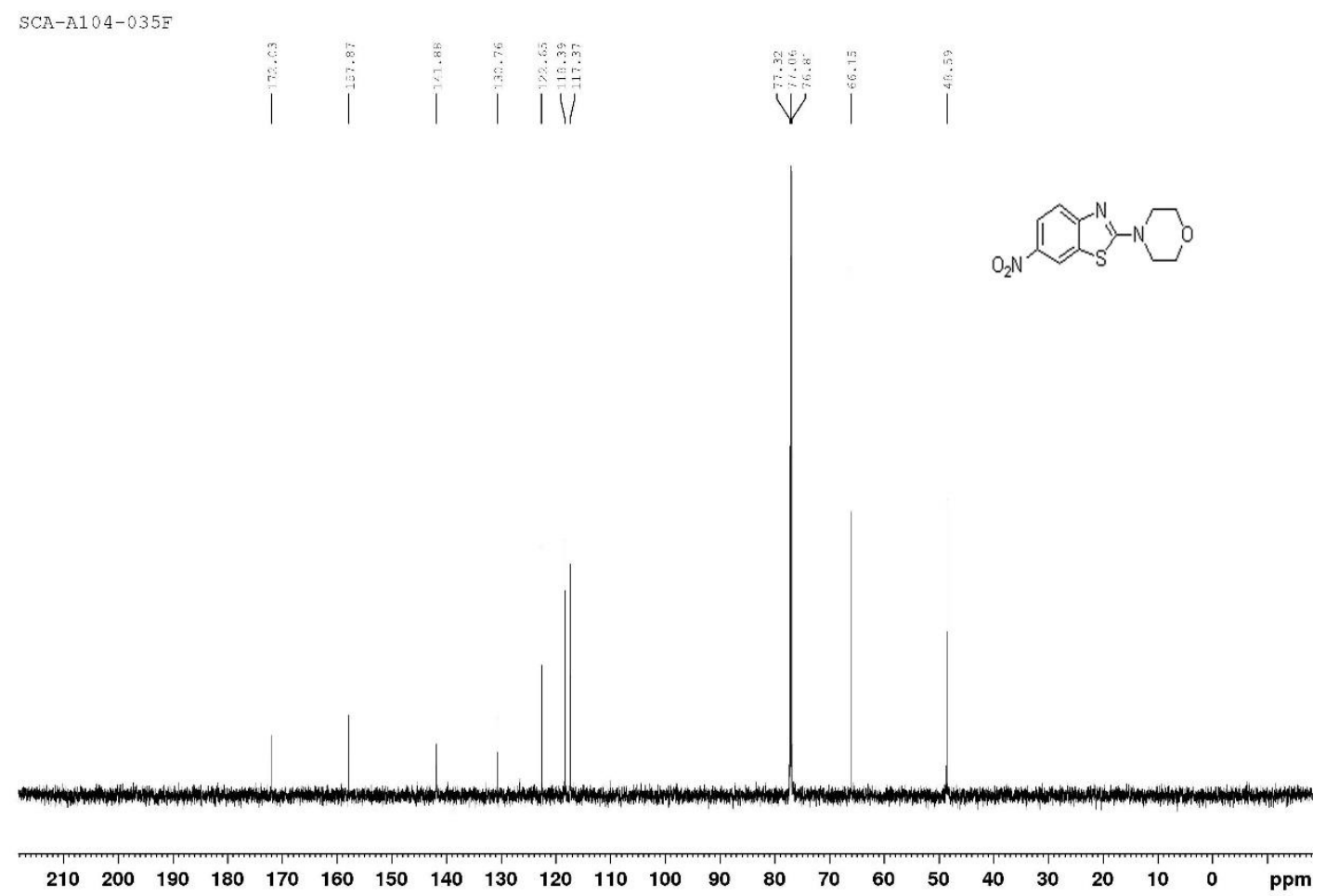

Figure S10: ${ }^{13} \mathrm{C}$ NMR spectra of4-(6-nitrobenzo[d]thiazol-2-yl)morpholine (9d). 


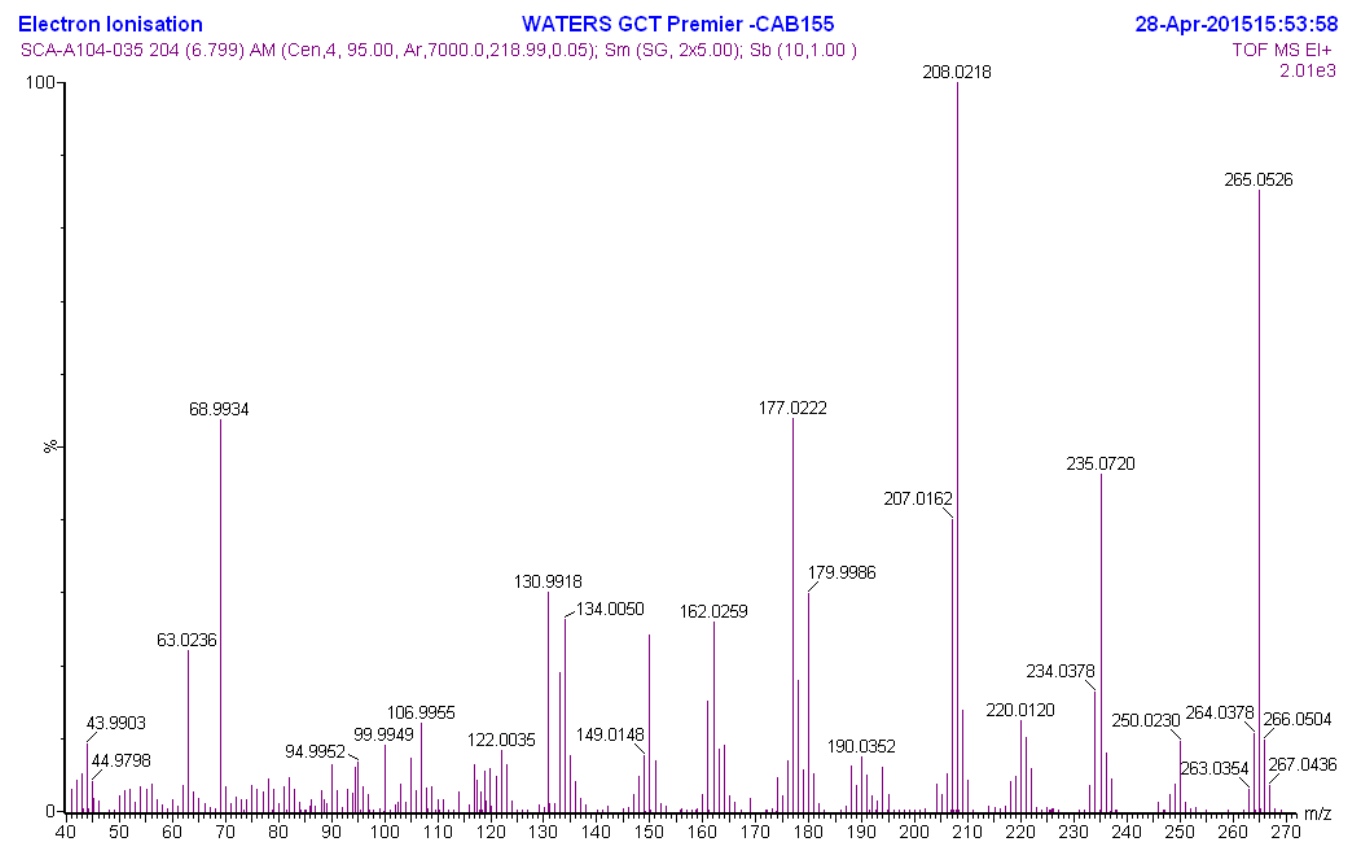

Figure S11: HRMS-EI spectra of 4-(6-nitrobenzo[d]thiazol-2-yl)morpholine (9d).

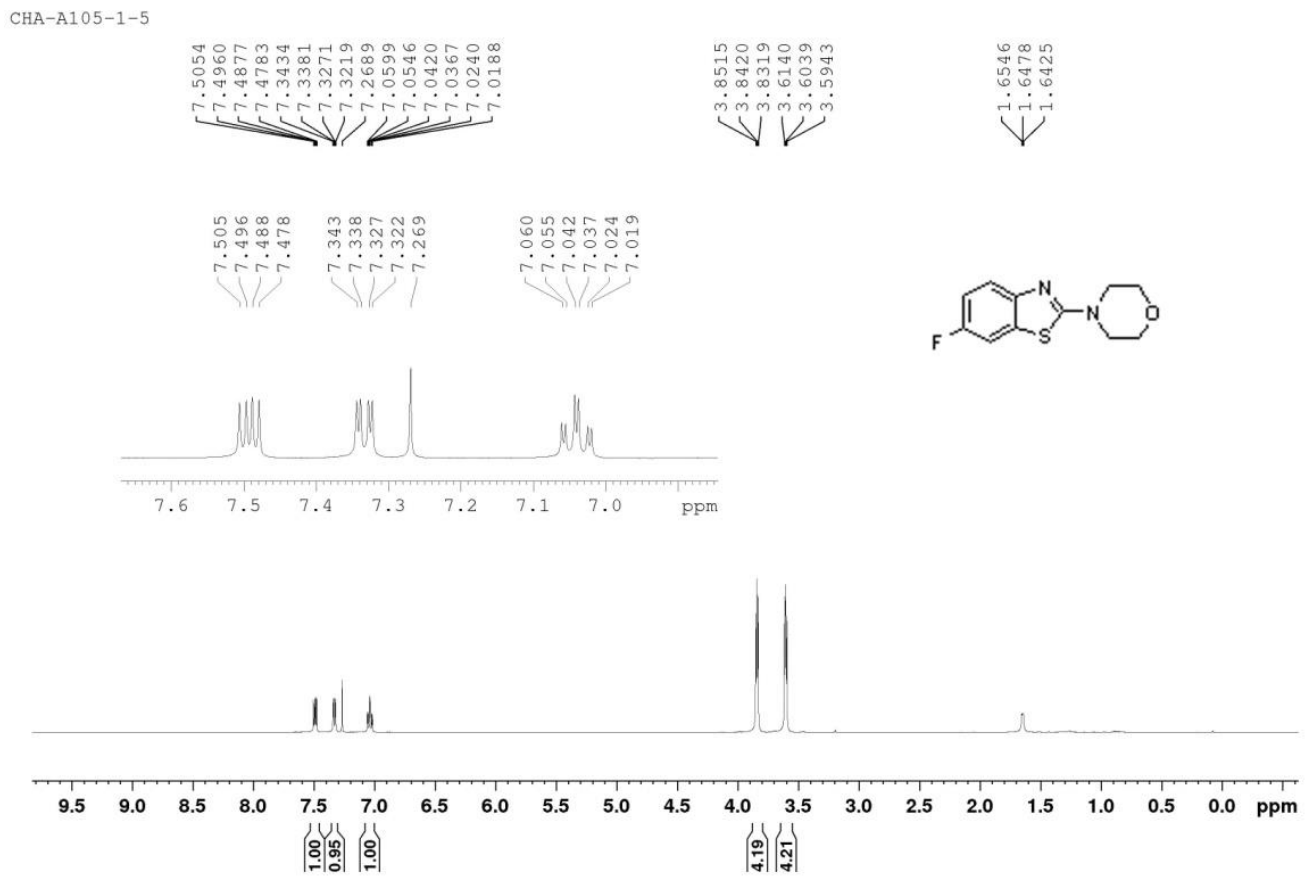

Figure S12: ${ }^{1} \mathrm{H}$ NMR spectra of 4-(6-fluorobenzo[d]thiazol-2-yl)morpholine (9e). 


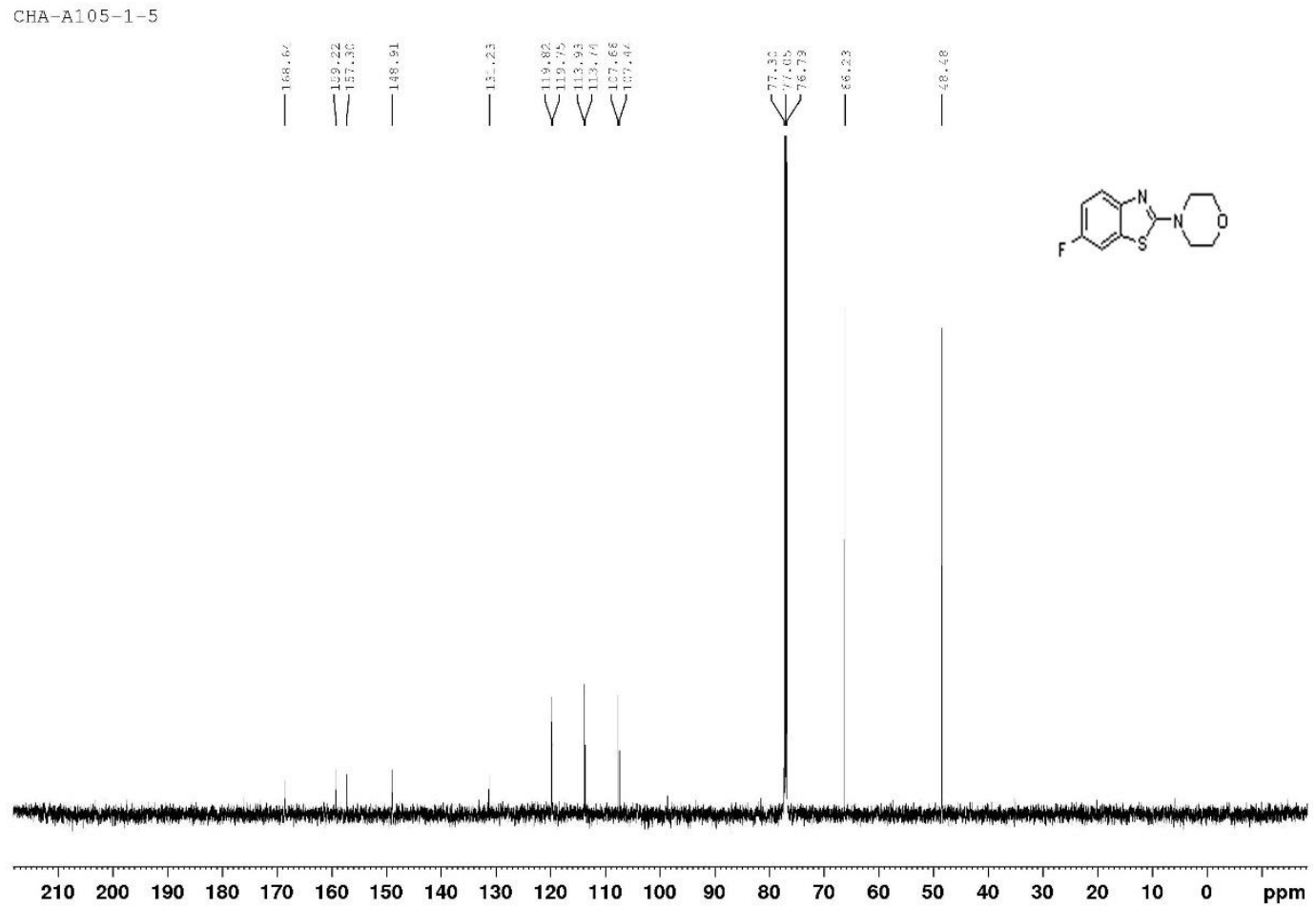

Figure S13: ${ }^{13} \mathrm{C}$ NMR spectra of 4-(6-fluorobenzo[d]thiazol-2-yl)morpholine (9e).

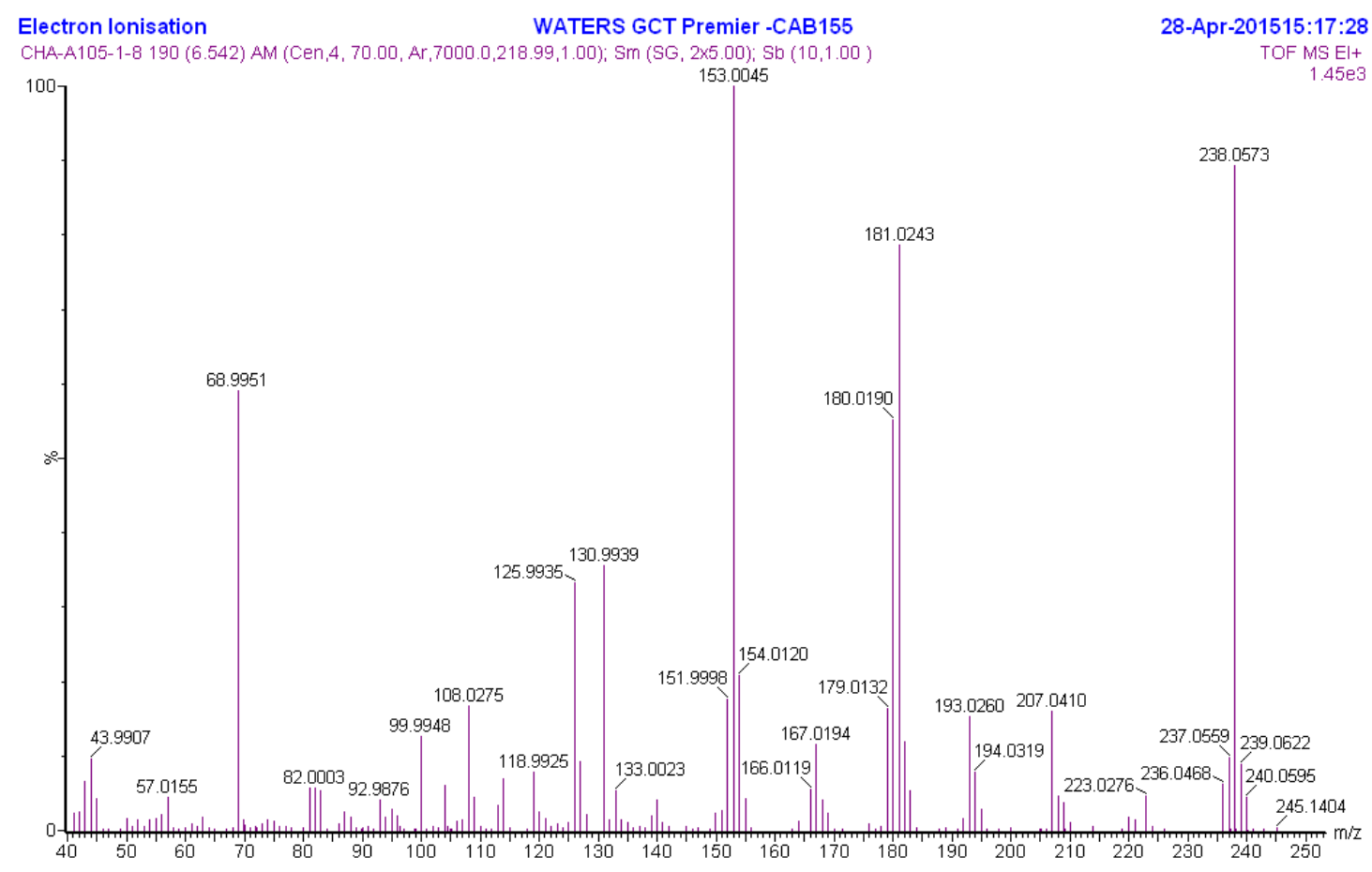

Figure S14: HRMS spectra of 4-(6-fluorobenzo[d]thiazol-2-yl)morpholine (9e). 


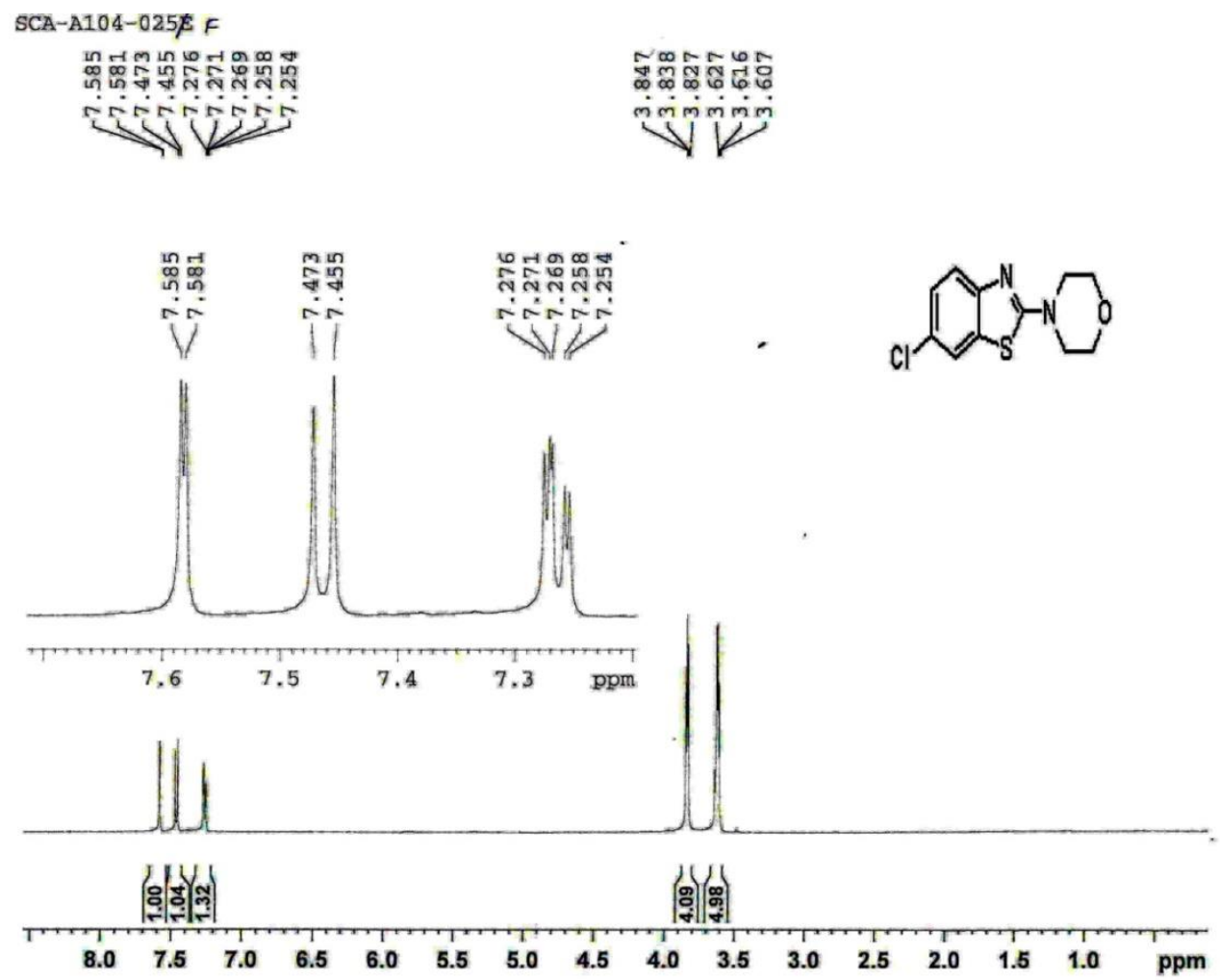

Figure S15: ${ }^{1} \mathrm{H}$ NMR spectra of 4-(6-chlorobenzo[d]thiazol-2-yl)morpholine (9f).

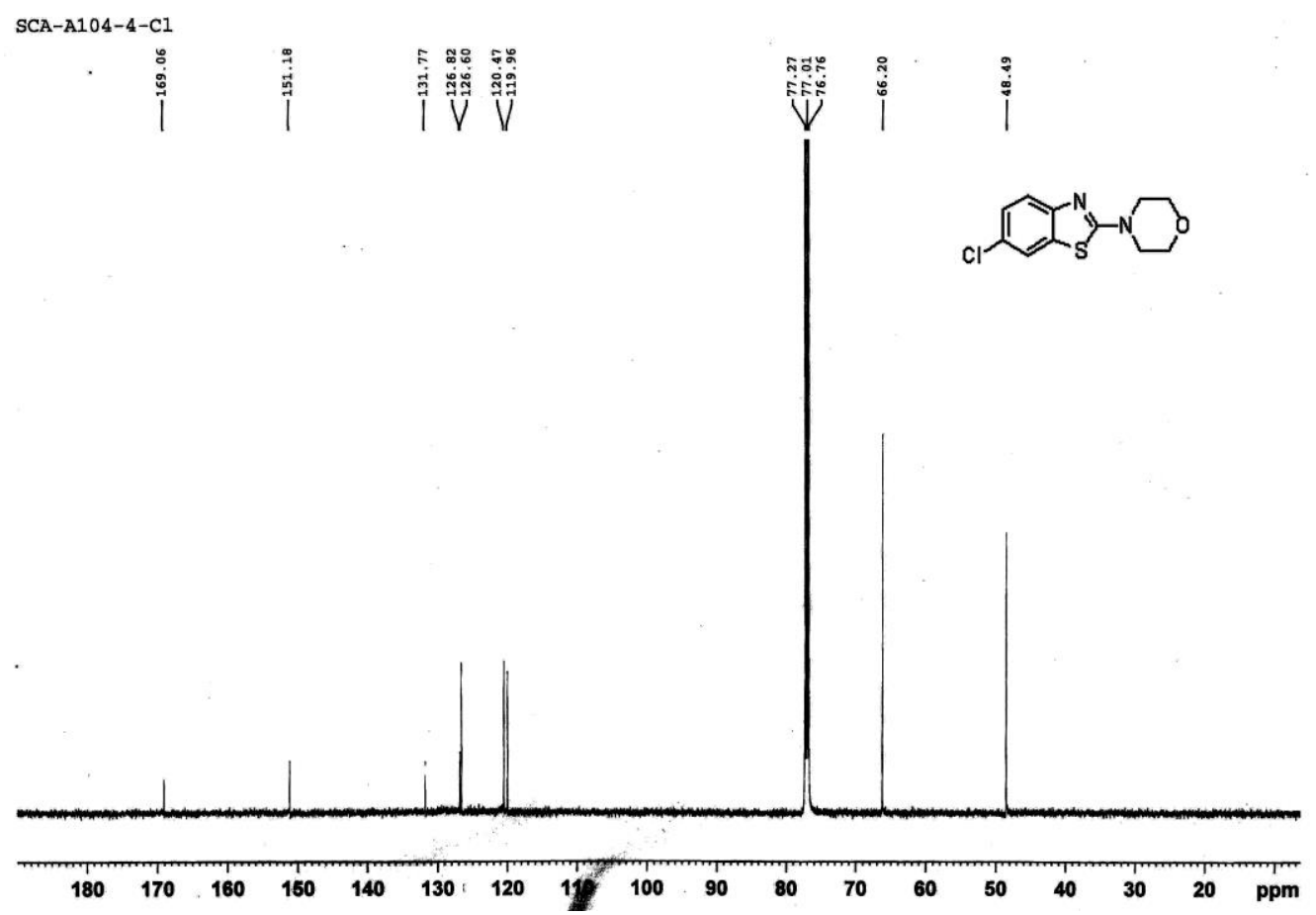

Figure S16: ${ }^{13}$ NMR spectra of 4-(6-chlorobenzo[d]thiazol-2-yl)morpholine (9f). 


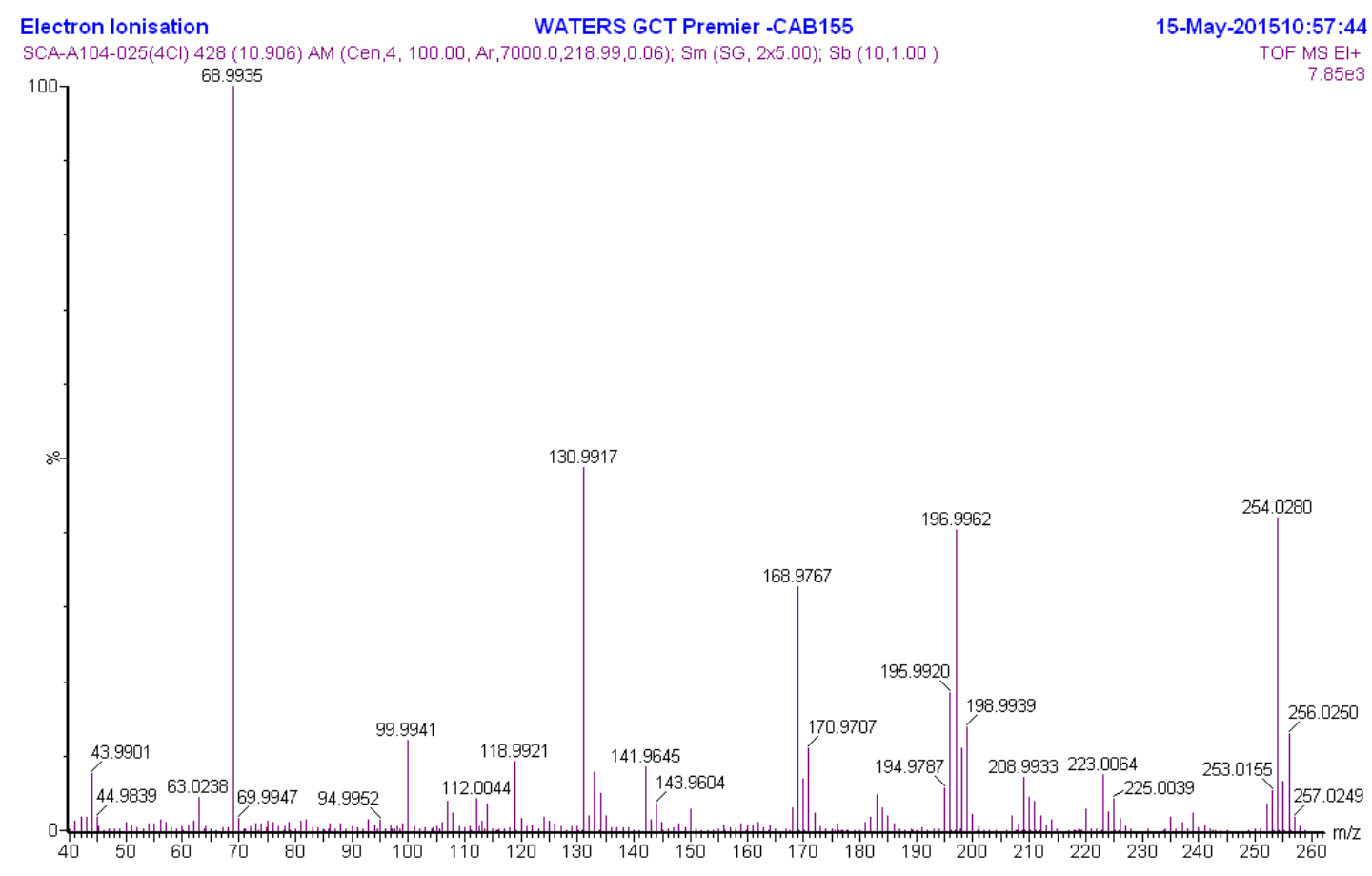

Figure S17: HRMS-EI spectra of 4-(6-chlorobenzo[d]thiazol-2-yl)morpholine(9f).

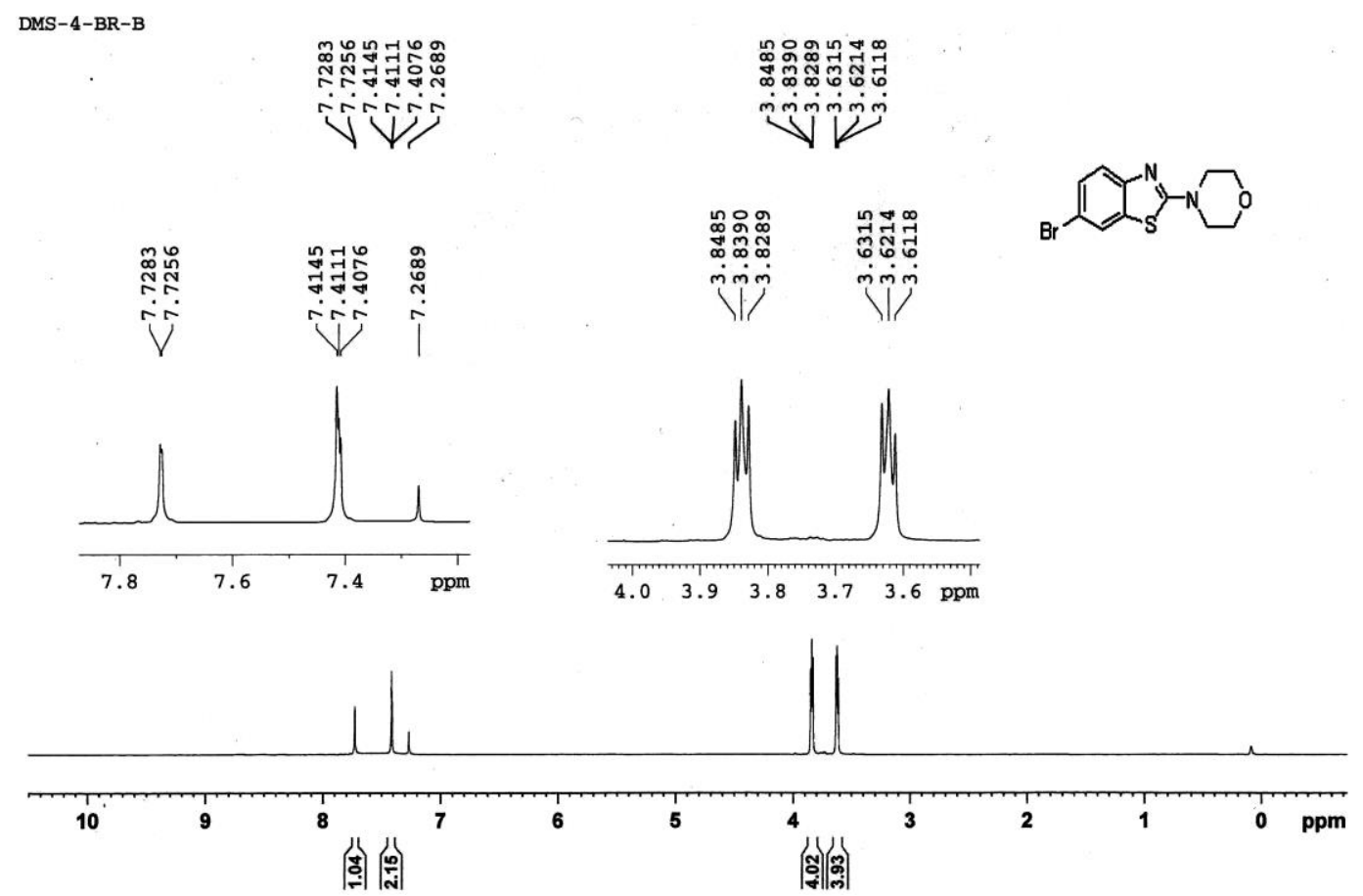

Figure S18: ${ }^{1} \mathrm{H}$ NMR spectra of 4-(6-bromobenzo[d]thiazol-2-yl)morpholine (9g). 


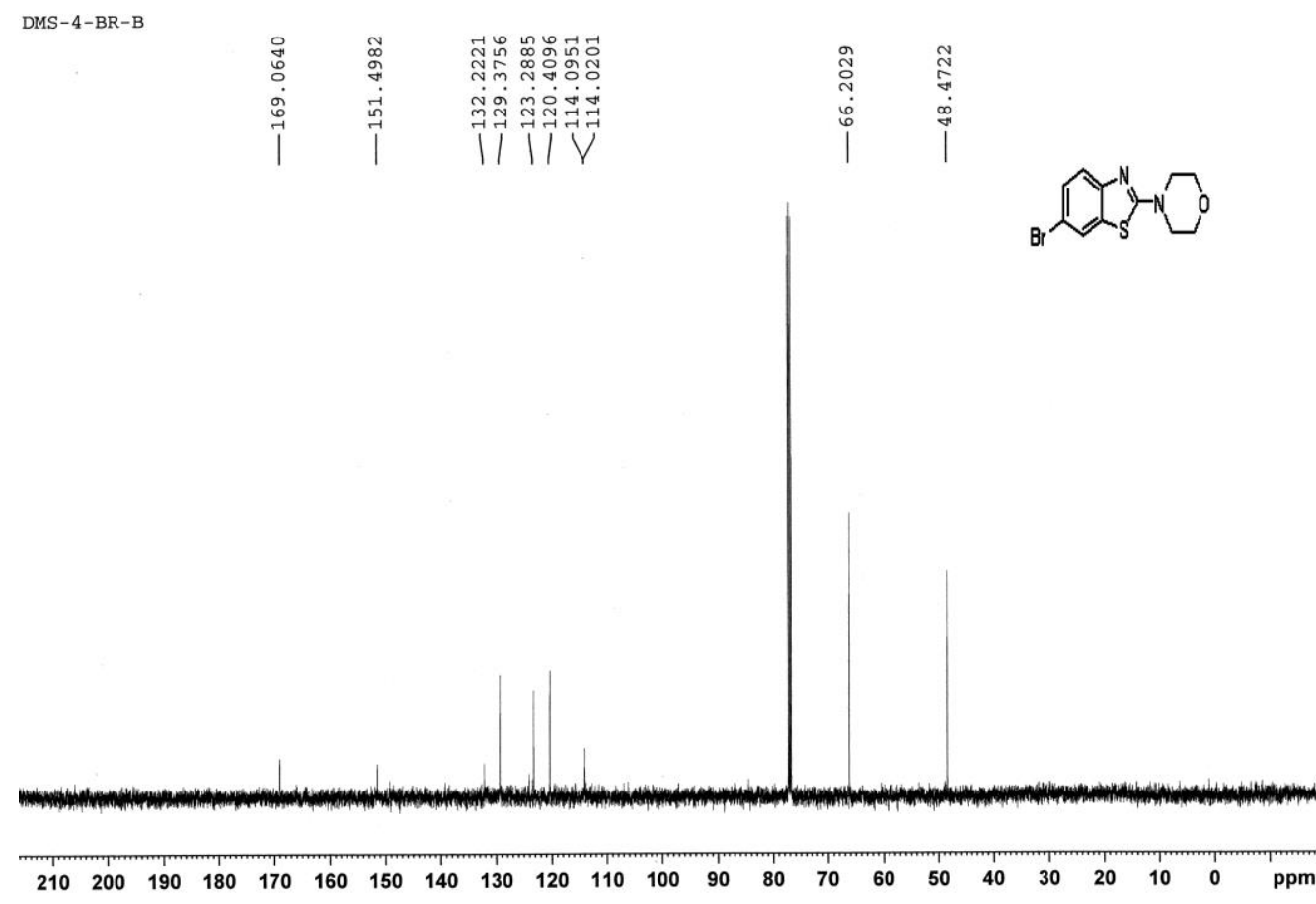

Figure S19: ${ }^{13} \mathrm{C}$ NMR spectra of 4-(6-bromobenzo[d]thiazol-2-yl)morpholine (9g).

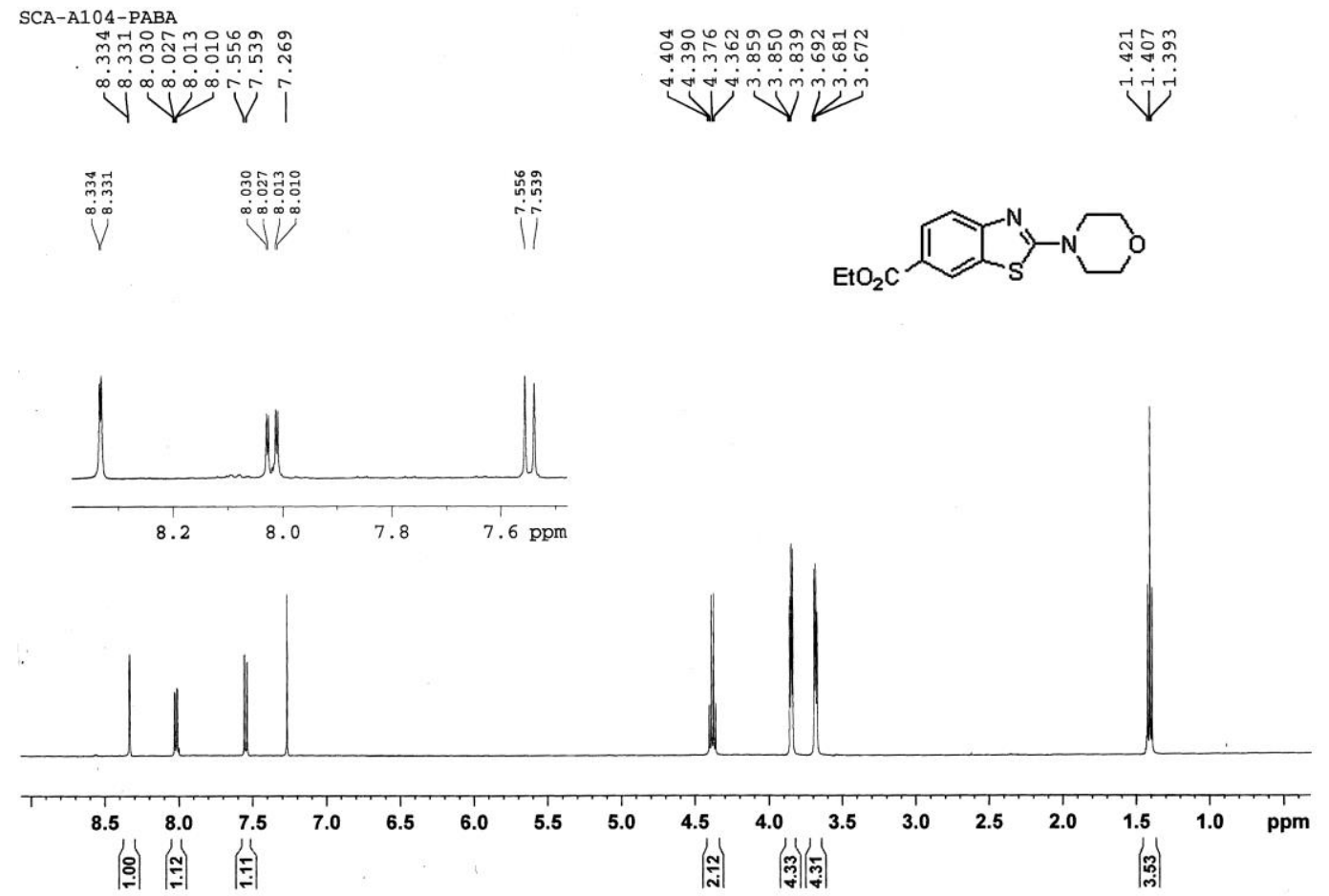

Figure S20: ${ }^{1} \mathrm{H}$ NMR spectra of methyl-2-morpholinobenzo[d]thiazole-6-carboxylate (9h). 


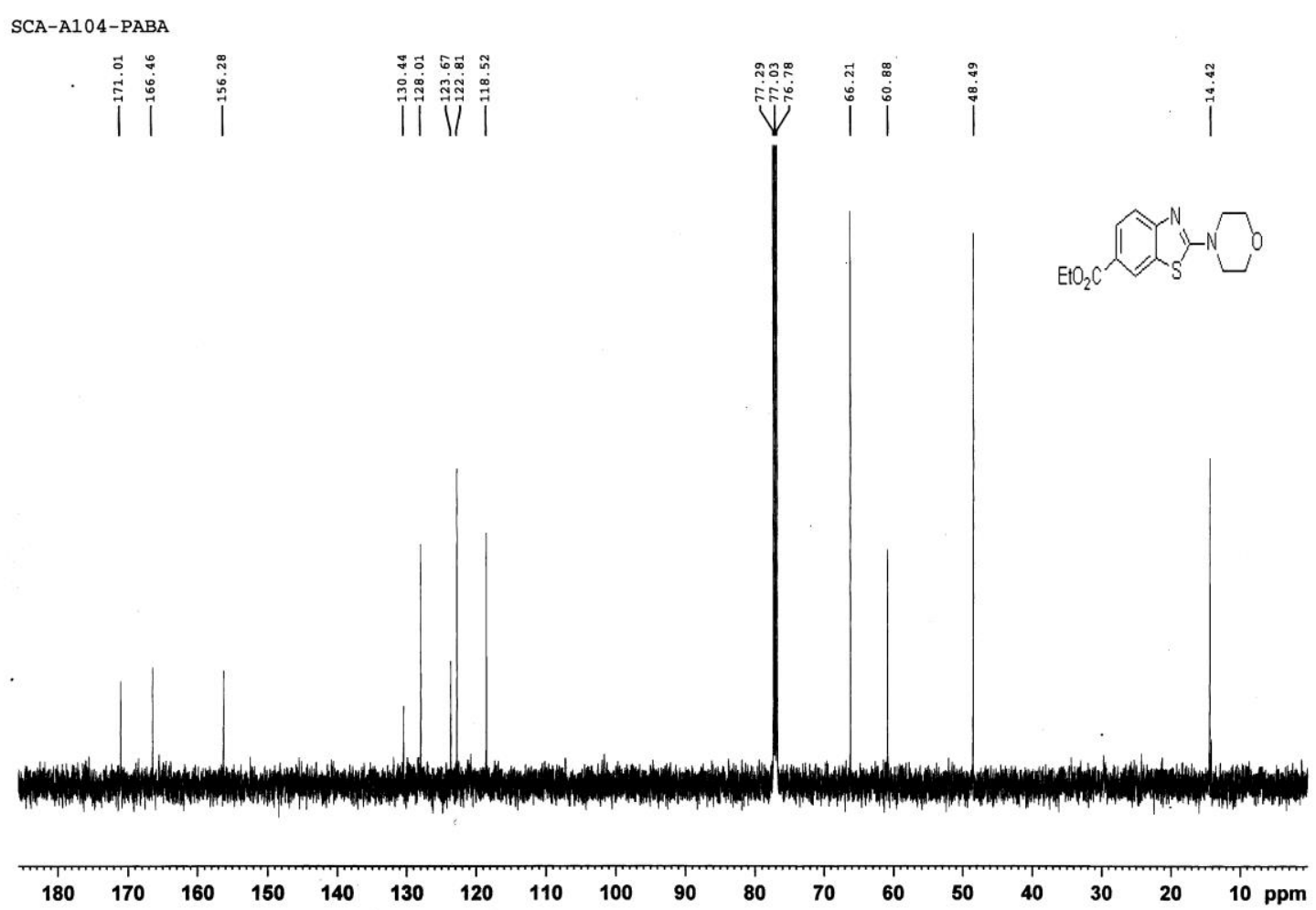

Figure S21: ${ }^{13} \mathrm{C}$ NMR spectra of methyl2-morpholinobenzo[d]thiazole-6-carboxylate $(\mathbf{9 h})$. SCA-A104-005
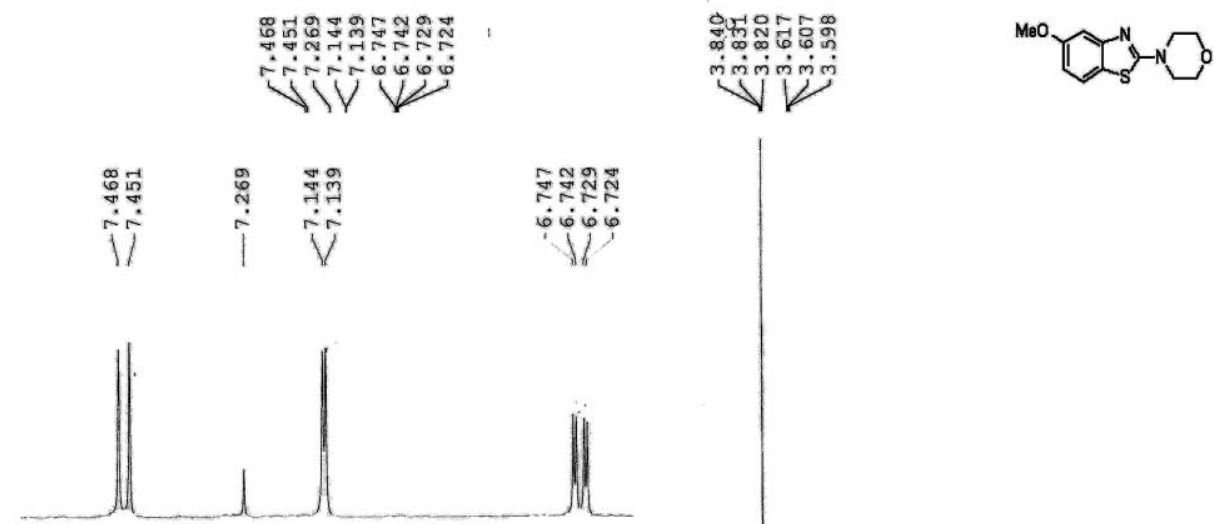

$\begin{array}{lllllllll}7.5 & 7.4 & 7.3 & 7.2 & 7.1 & 7.0 & 6.9 & 6.8\end{array}$

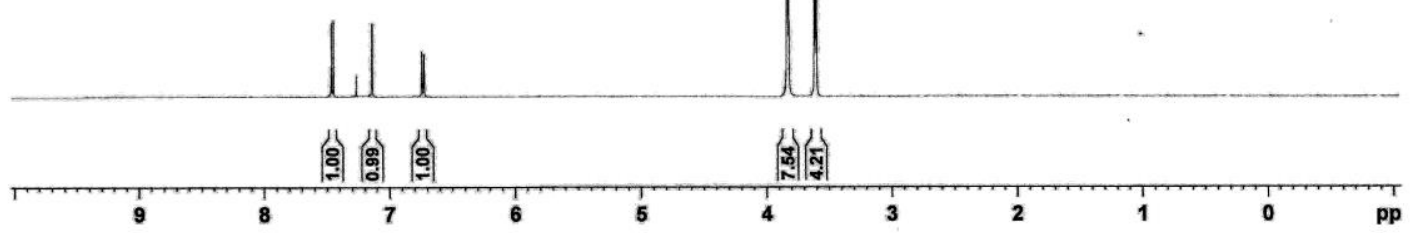

Figure S22: ${ }^{1}$ H NMR spectra of4-(5-methoxybenzo[d]thiazol-2-yl)morpholine (9i). 

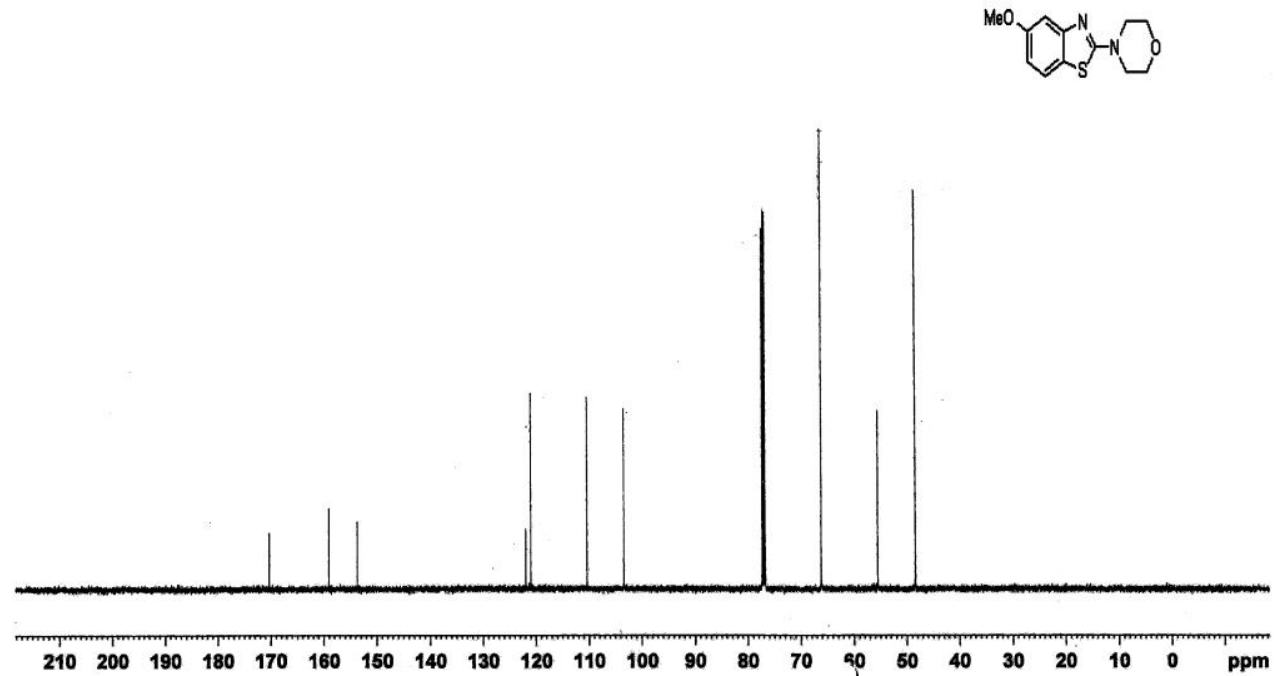

Figure S23: ${ }^{13} \mathrm{C}$ NMR spectra of4-(5-methoxybenzo[d]thiazol-2-yl)morpholine (9i).

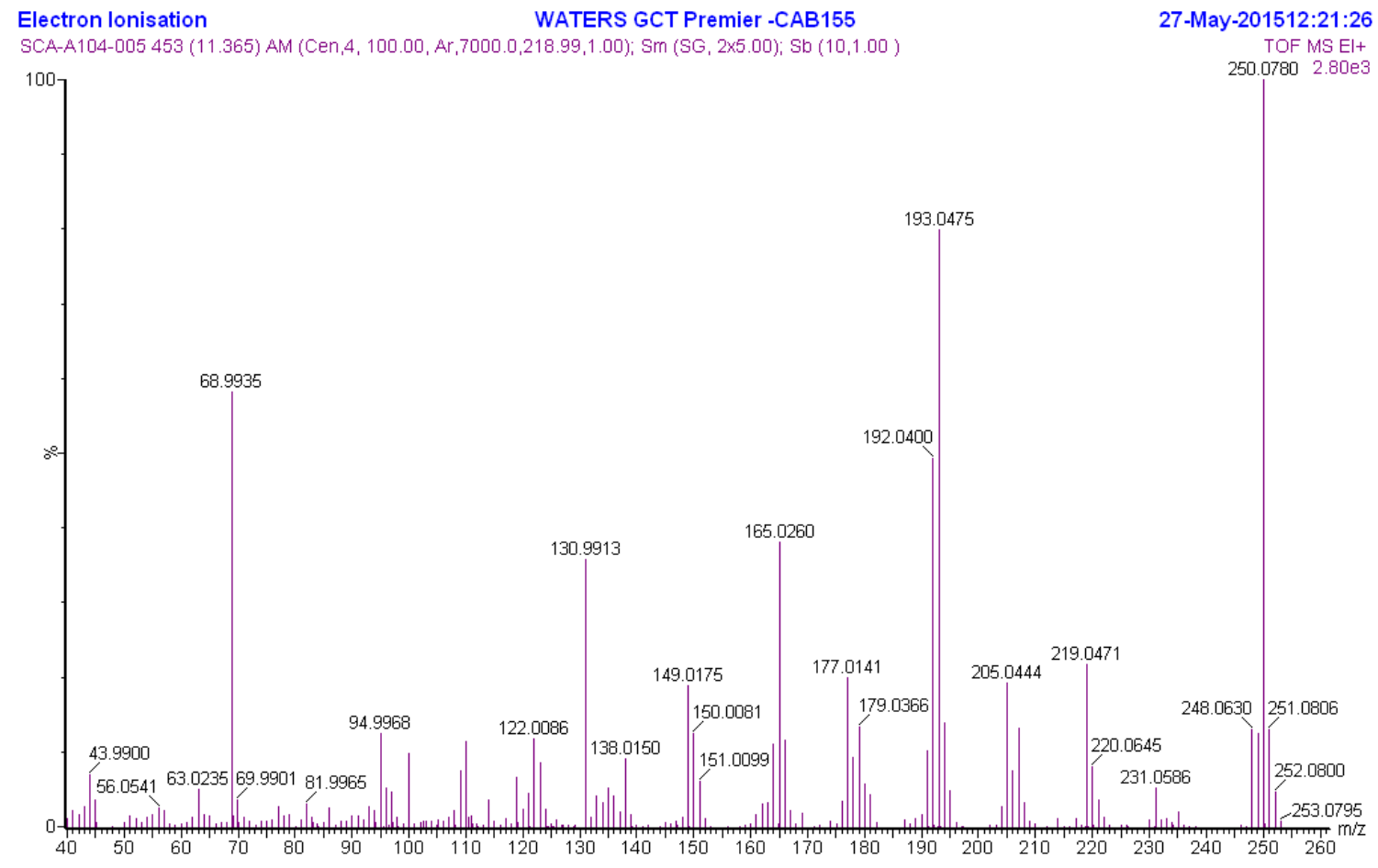

Figure S24: HRMS-EI spectra of 4-(5-methoxybenzo[d]thiazol-2-yl)morpholine (9i). 

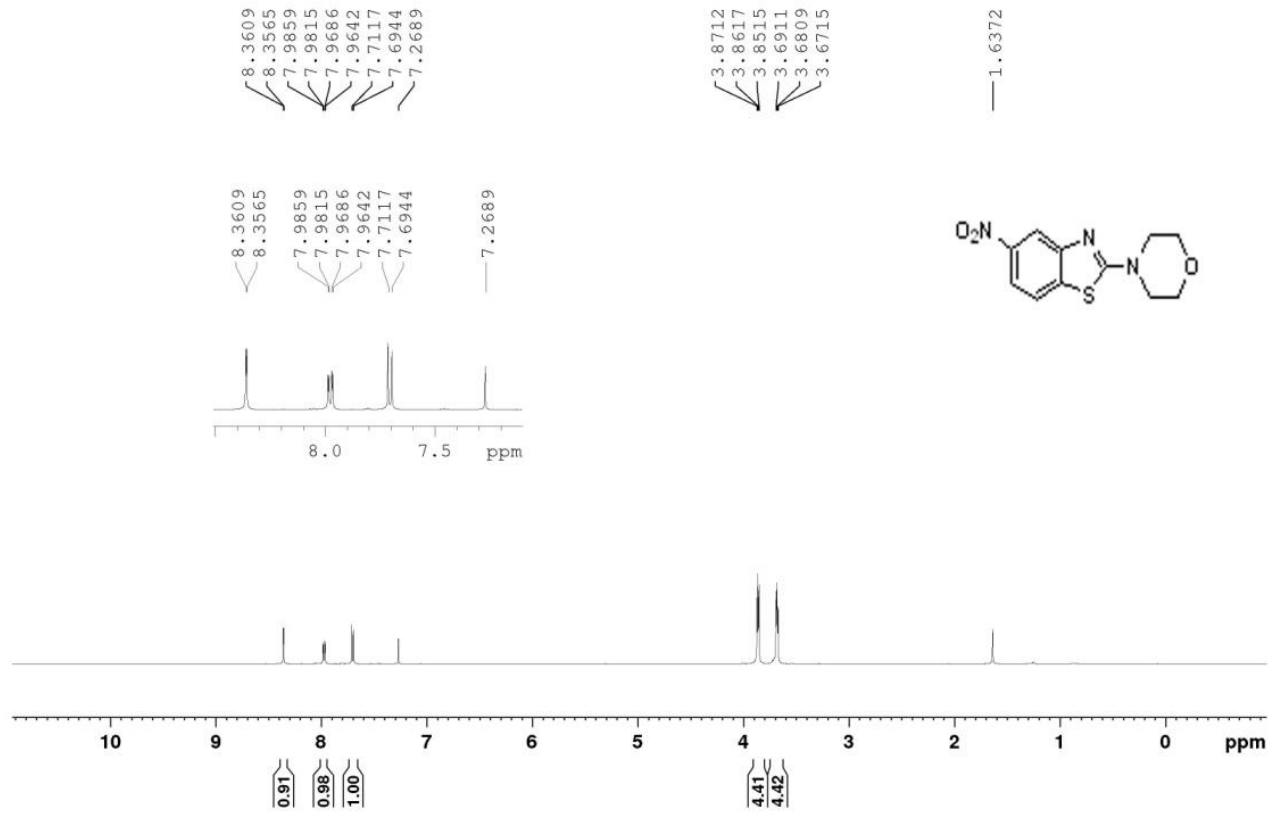

Figure S25: ${ }^{1} \mathrm{H}$ NMR spectra of4-(5-nitrobenzo[d]thiazol-2-yl)morpholine $(\mathbf{9 j})$.

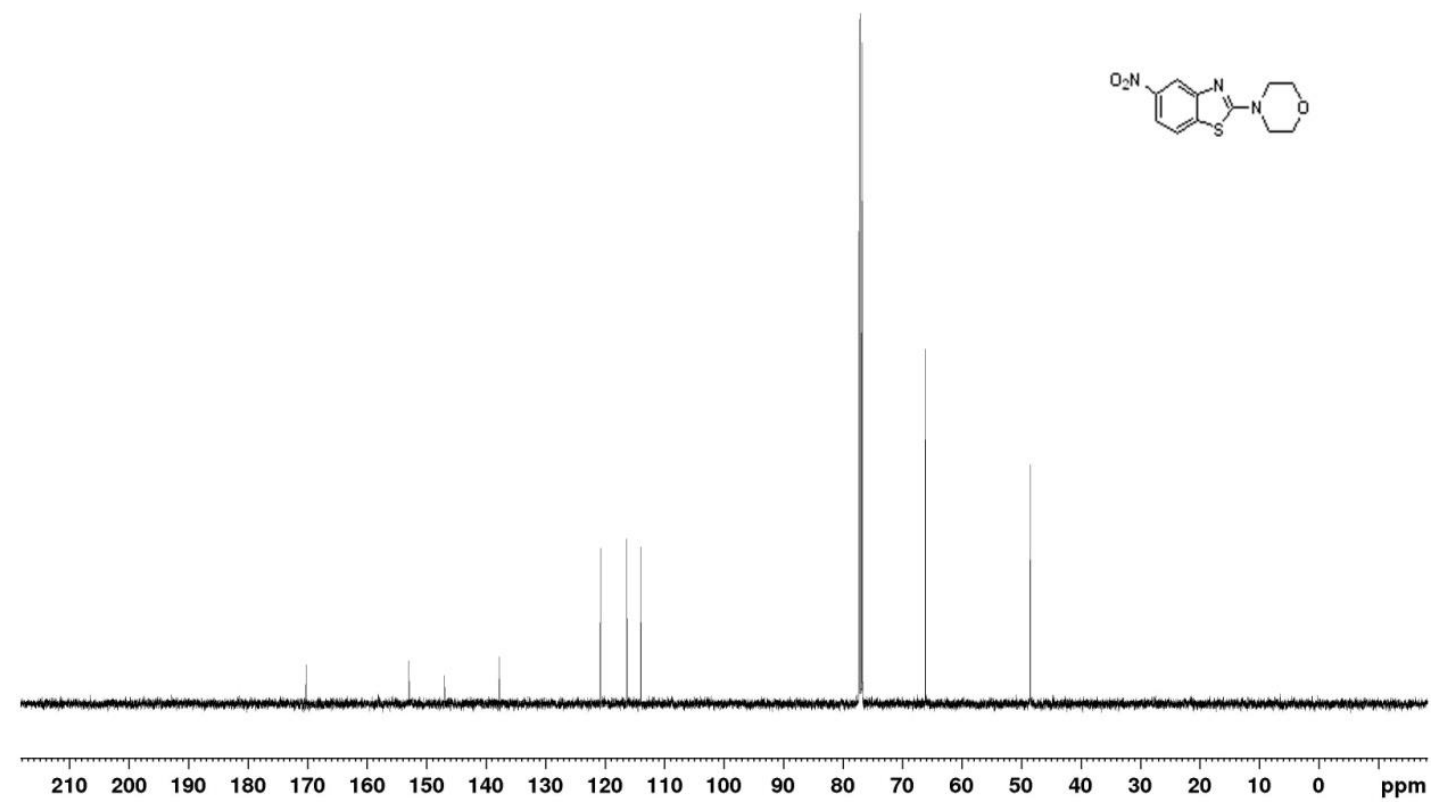

Figure S26: ${ }^{13} \mathrm{C}$ NMR spectra of4-(5-nitrobenzo[d]thiazol-2-yl)morpholine(9j). 


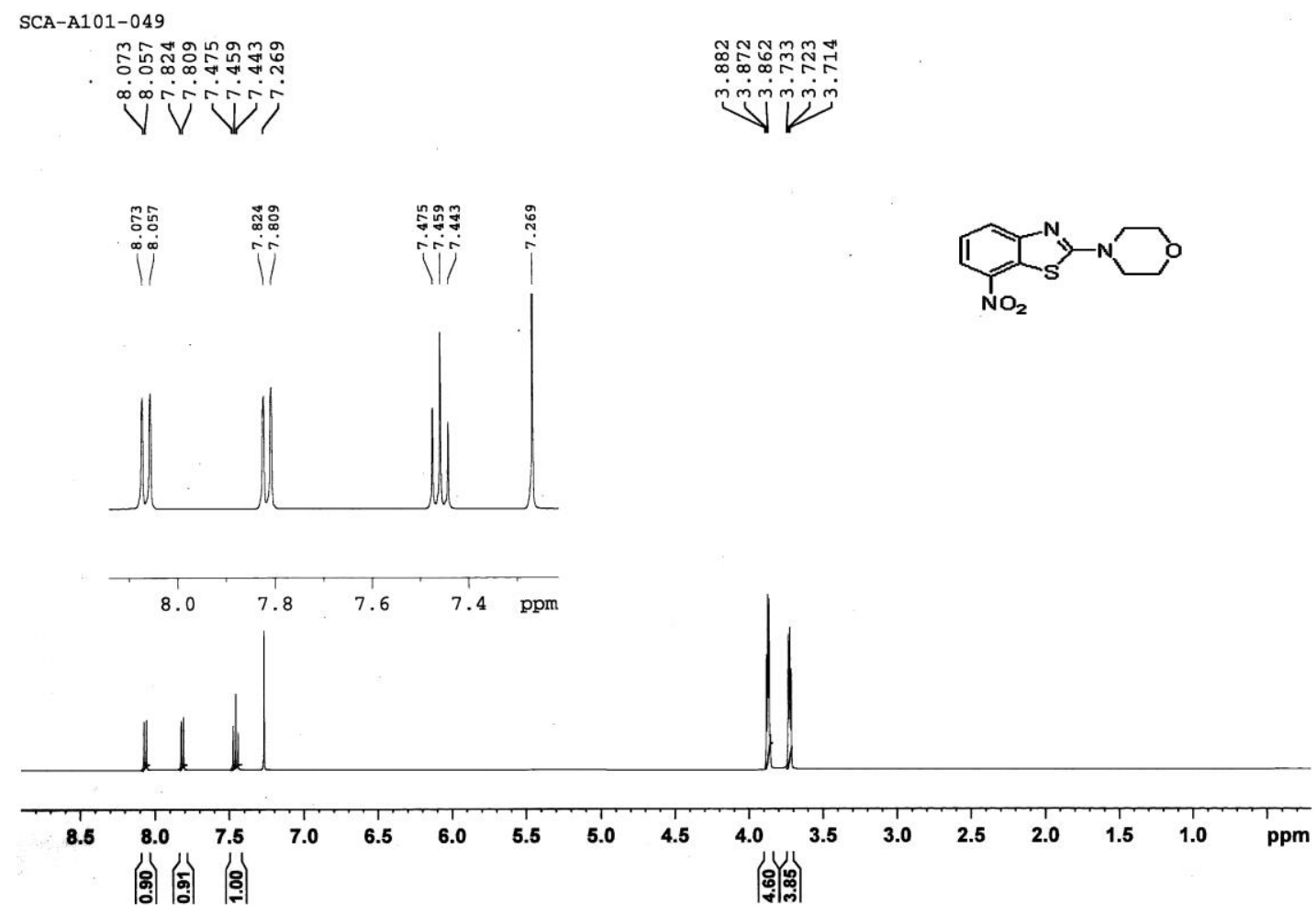

Figure S27: ${ }^{1}$ H NMR spectra of 4-(7-nitrobenzo[d]thiazol-2-yl)morpholine (9k).

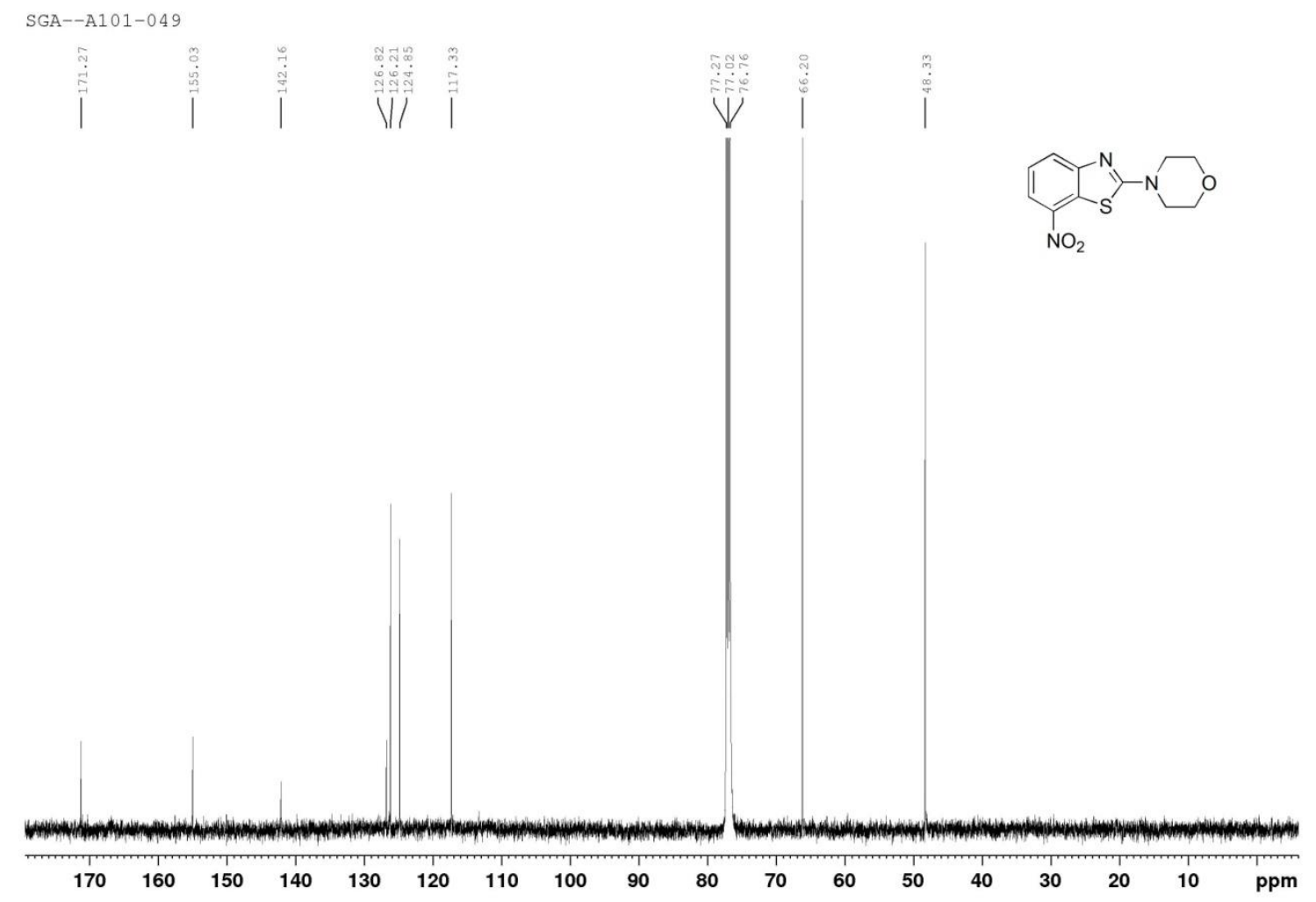

Figure S28: ${ }^{13} \mathrm{C}$ NMR spectra of 4-(7-nitrobenzo[d]thiazol-2-yl)morpholine (9k). 


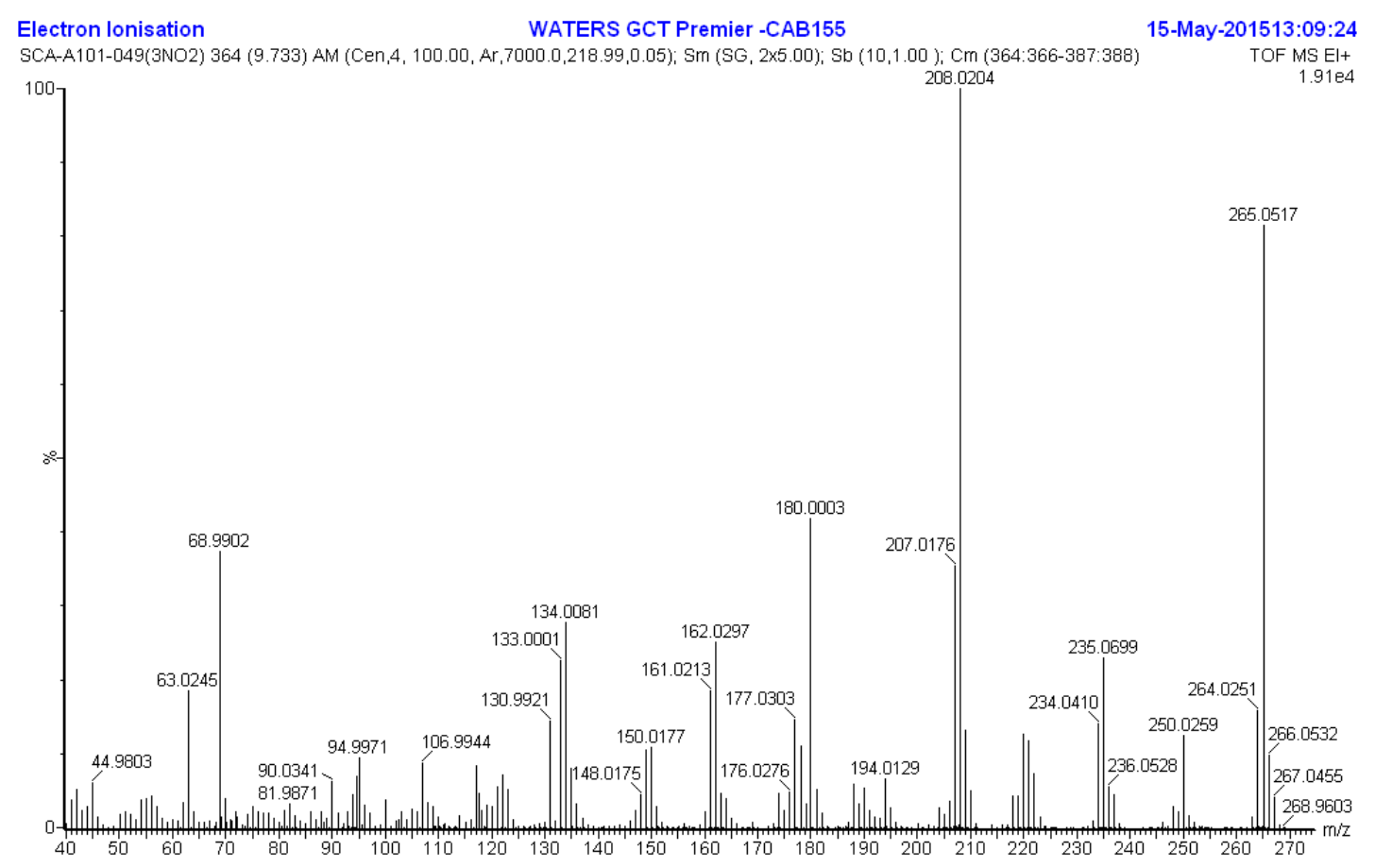

Figure S29: HRMS-EI spectra of 4-(7-nitrobenzo[d]thiazol-2-yl)morpholine (9k).

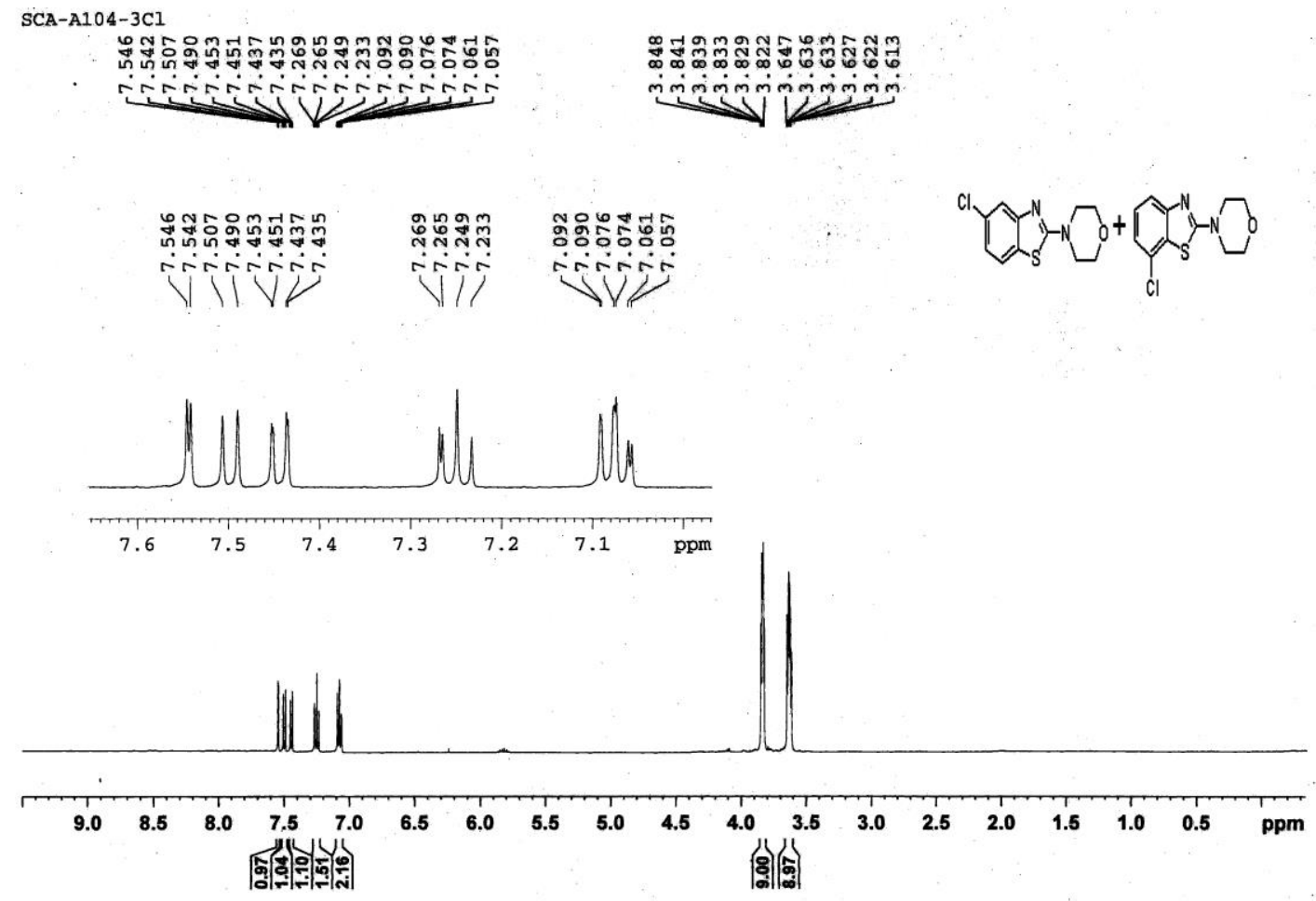

Figure S30: ${ }^{1} \mathrm{H}$ NMR spectra ofmixture of 4-(5-chlorobenzo[d]thiazol-2-yl)morpholine (9l) and 4-(7-chlorobenzo[d]thiazol-2-yl)morpholine (9m). 


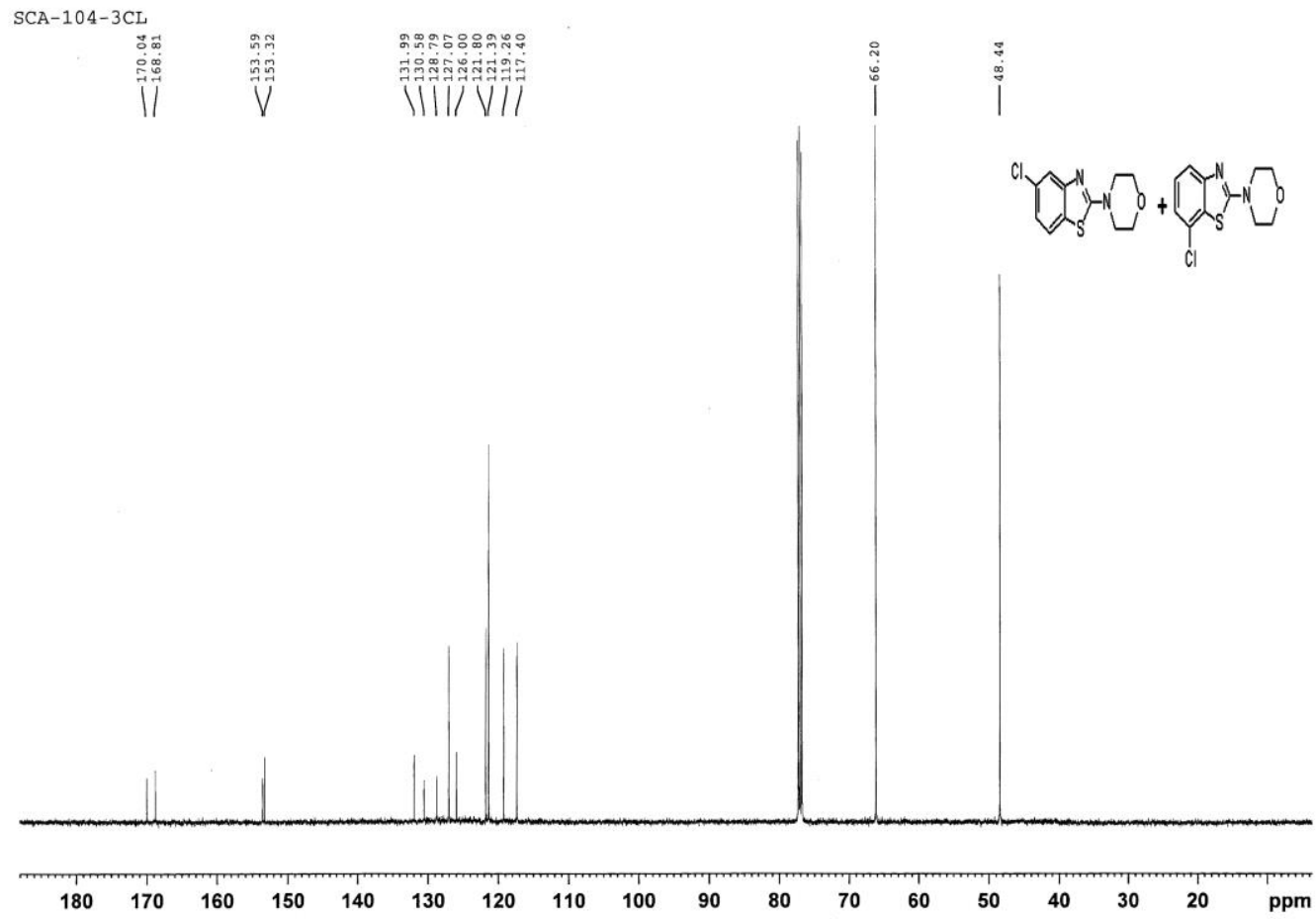

Figure 31: ${ }^{13} \mathrm{C}$ NMR spectra of mixture of 4-(5-chlorobenzo[d]thiazol-2-yl)morpholine (9l) and 4-(7-chlorobenzo[d]thiazol-2-yl)morpholine (9m).

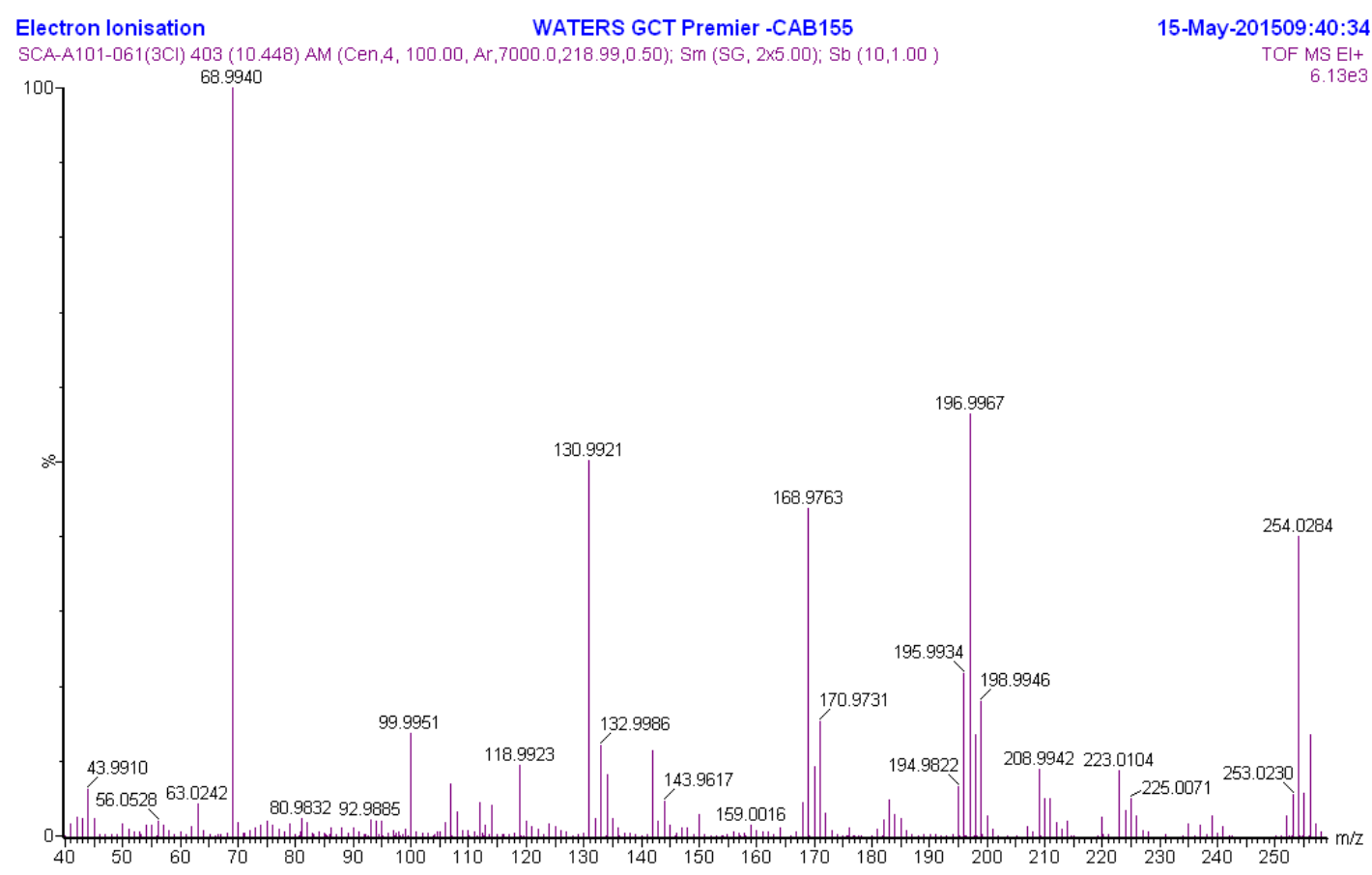

Figure S32: HRMS-EI spectra of mixture of 4-(5-chlorobenzo[d]thiazol-2-yl)morpholine (9l) and 4-(7-chlorobenzo[d]thiazol-2-yl)morpholine (9m). 

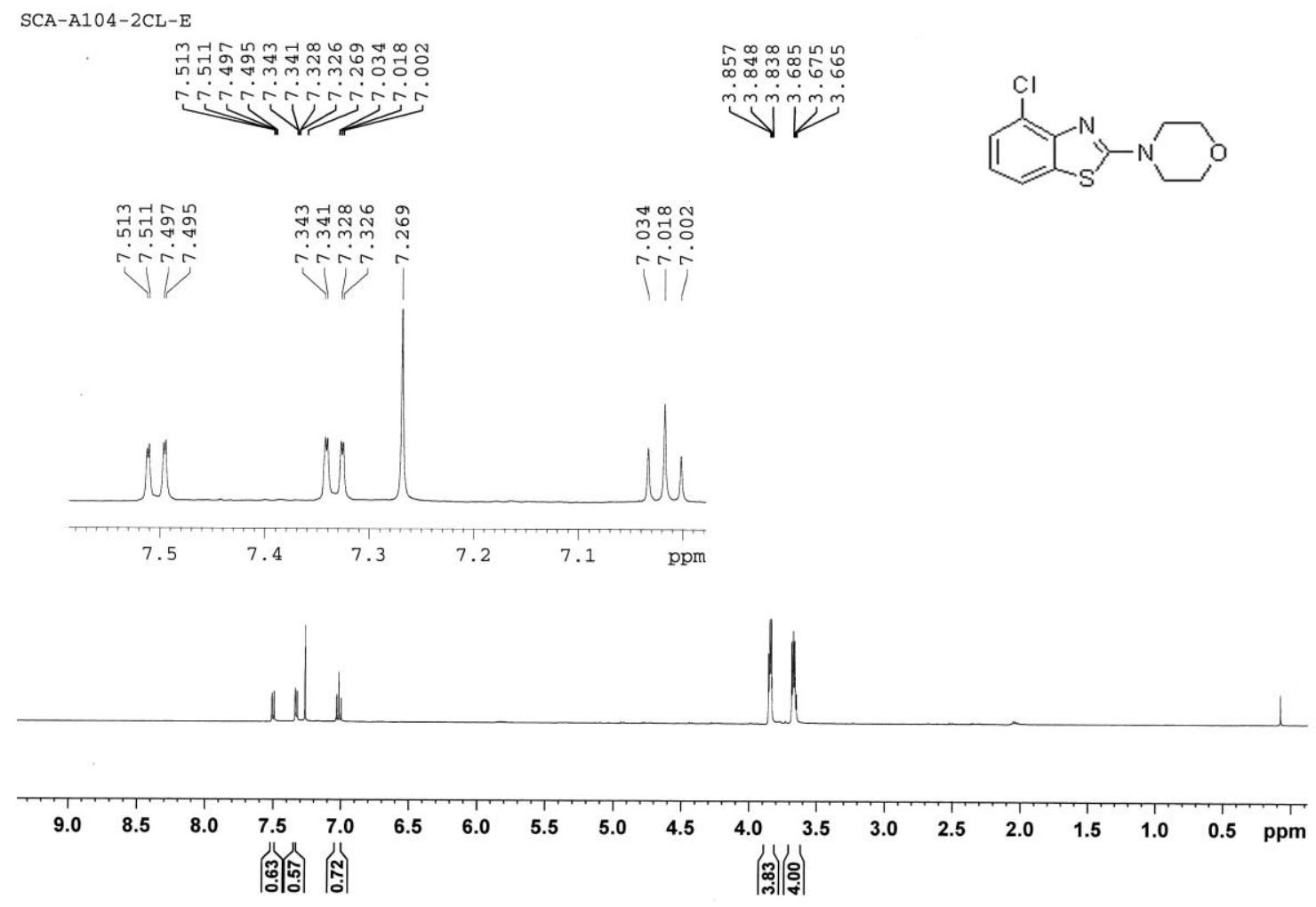

Figure S33: ${ }^{1}$ H NMR spectra of 4-(4-chlorobenzo[d]thiazol-2-yl)morpholine (9n).

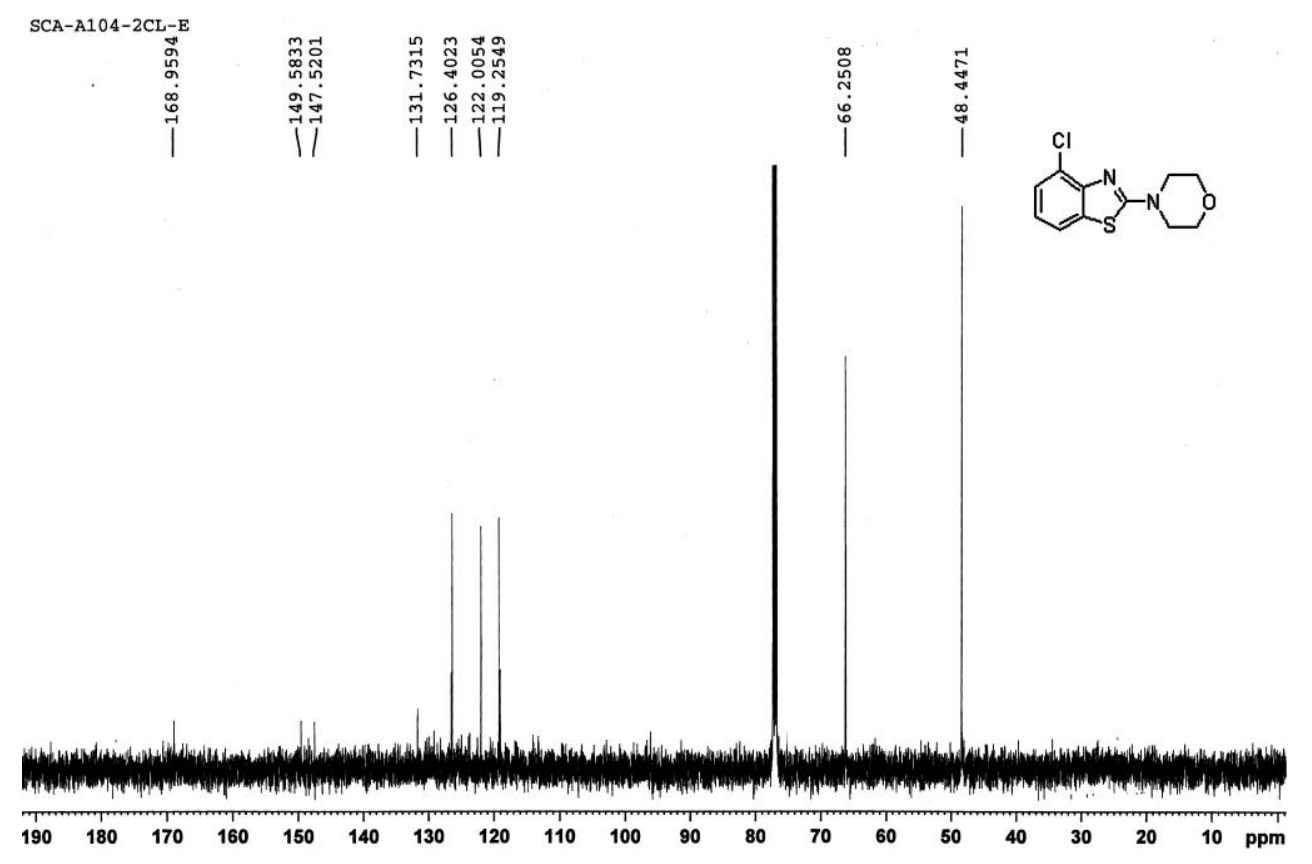

Figure 34: ${ }^{13} \mathrm{C}$ NMR spectra of 4-(4-chlorobenzo[d]thiazol-2-yl)morpholine (9n). 


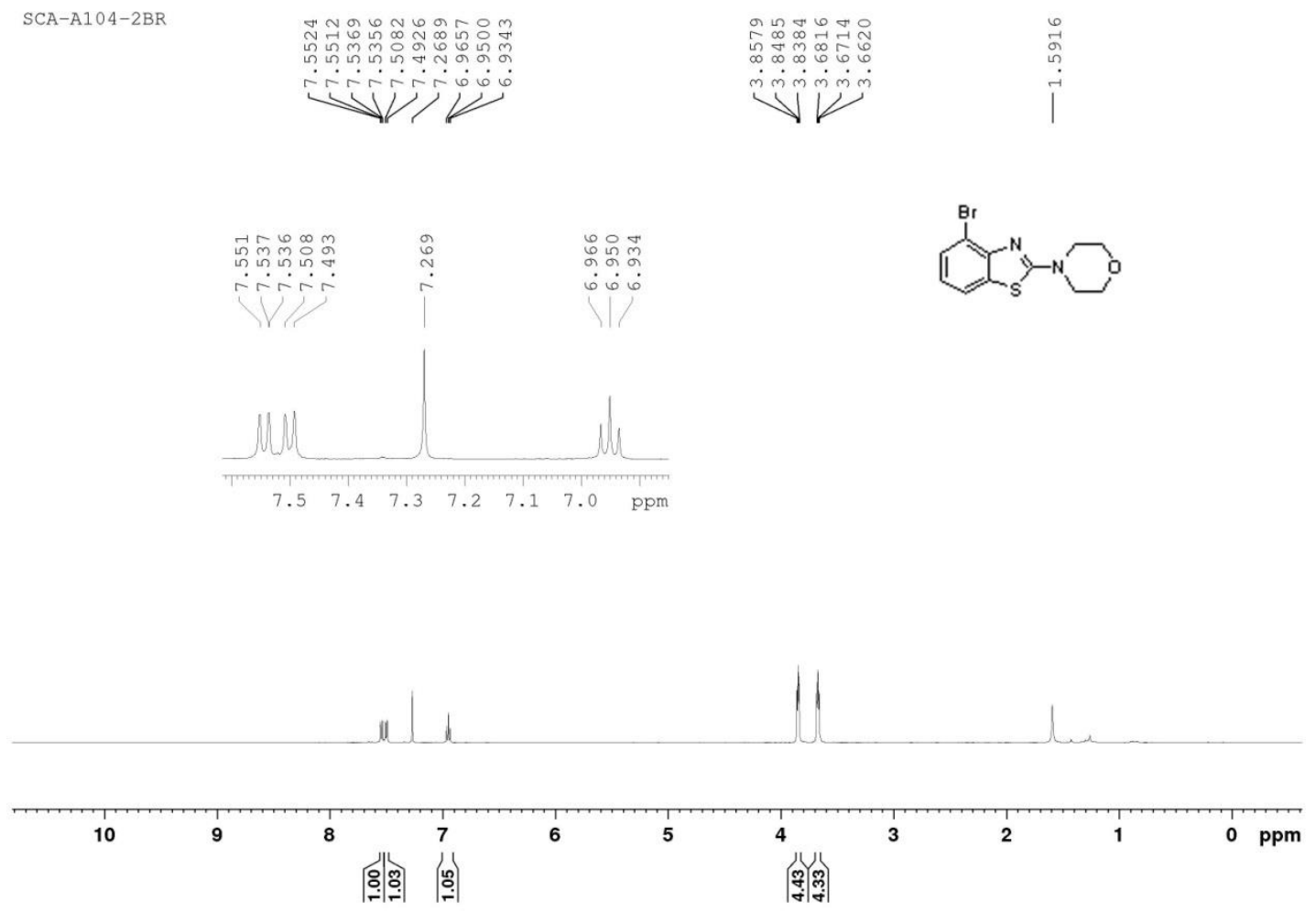

Figure 35: ${ }^{1} \mathrm{H}$ NMR spectra of 4-(4-bromobenzo[d]thiazol-2-yl)morpholine (9o).

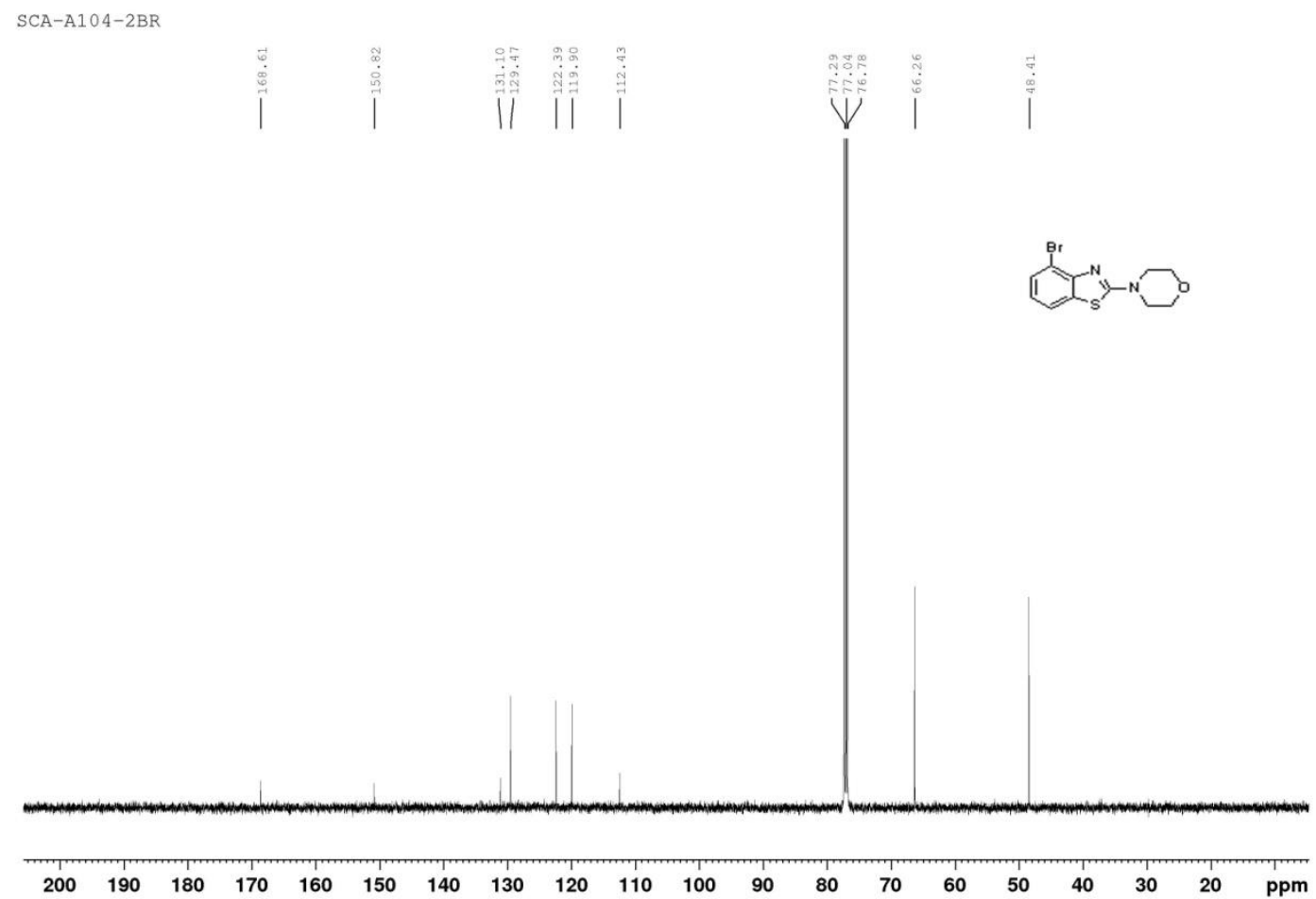

Figure 36: ${ }^{13} \mathrm{C}$ NMR spectra of4-(4-bromobenzo[d]thiazol-2-yl)morpholine (9o). 


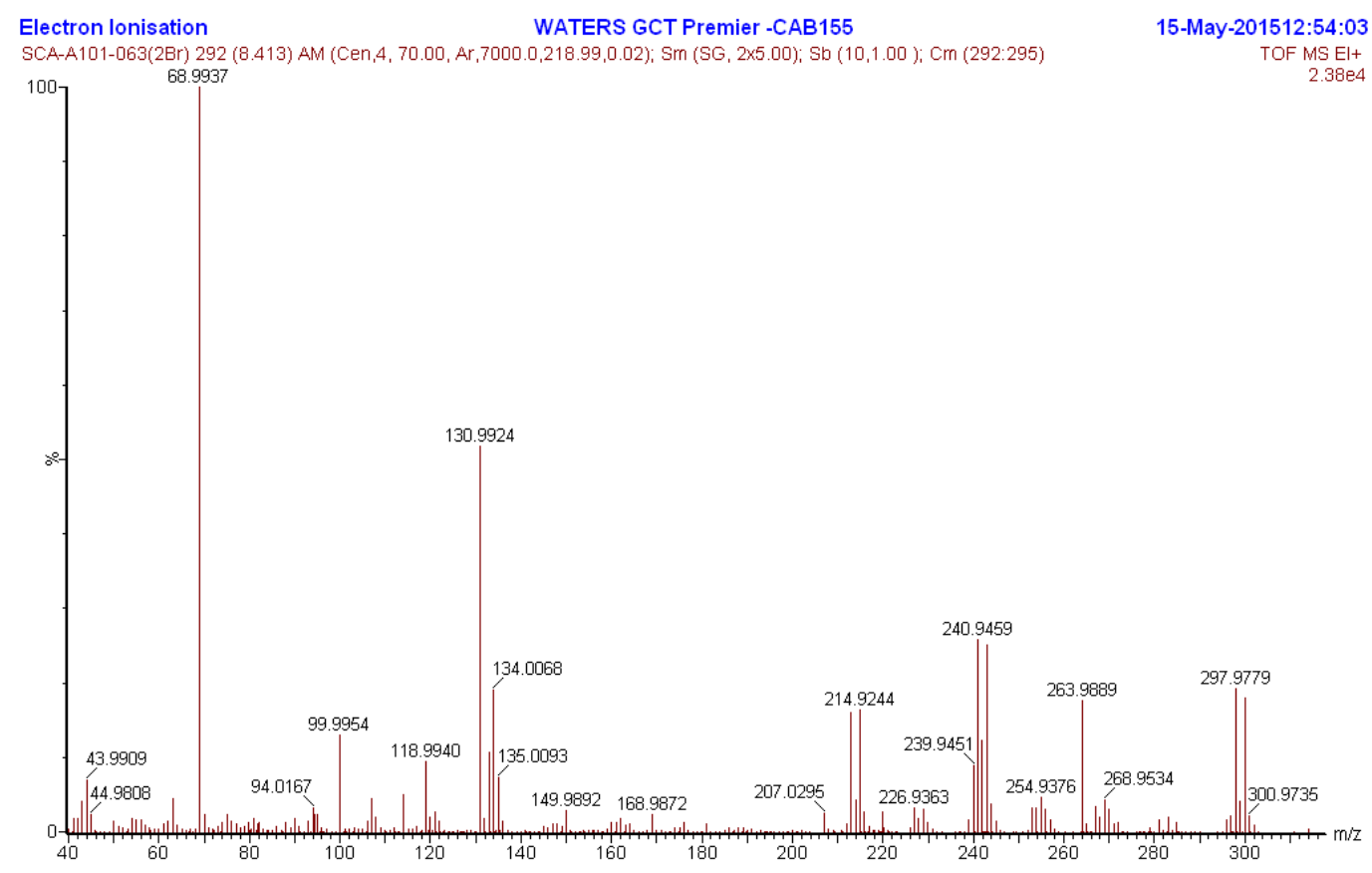

Figure 37: HRMS-EI spectra of 4-(4-bromobenzo[d]thiazol-2-yl)morpholine (9o).

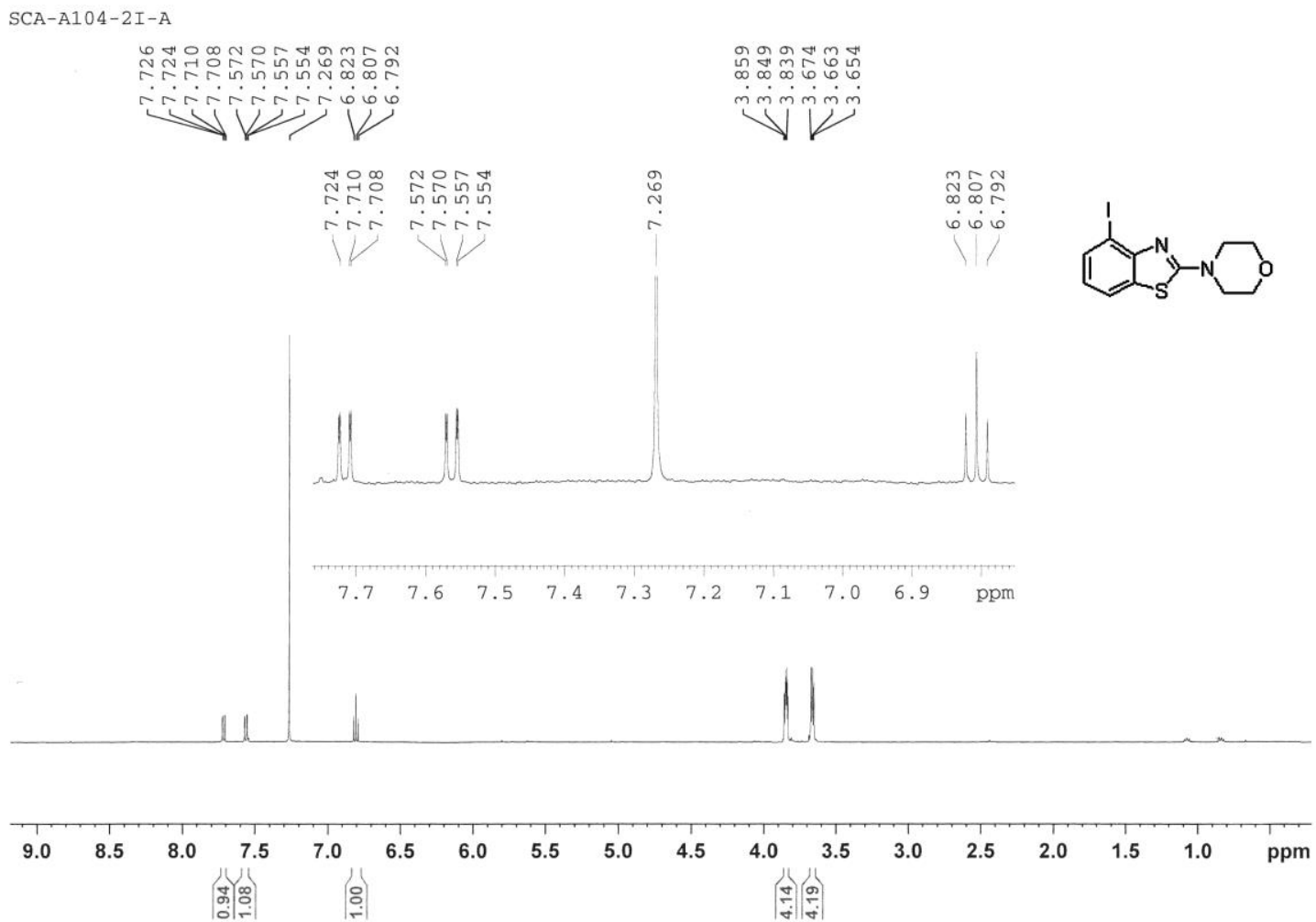

Figure 38: ${ }^{1} \mathrm{H}$ NMR spectra of 4-(4-iodobenzo[d]thiazol-2-yl)morpholine (9p). 
SCA-A104-2I A
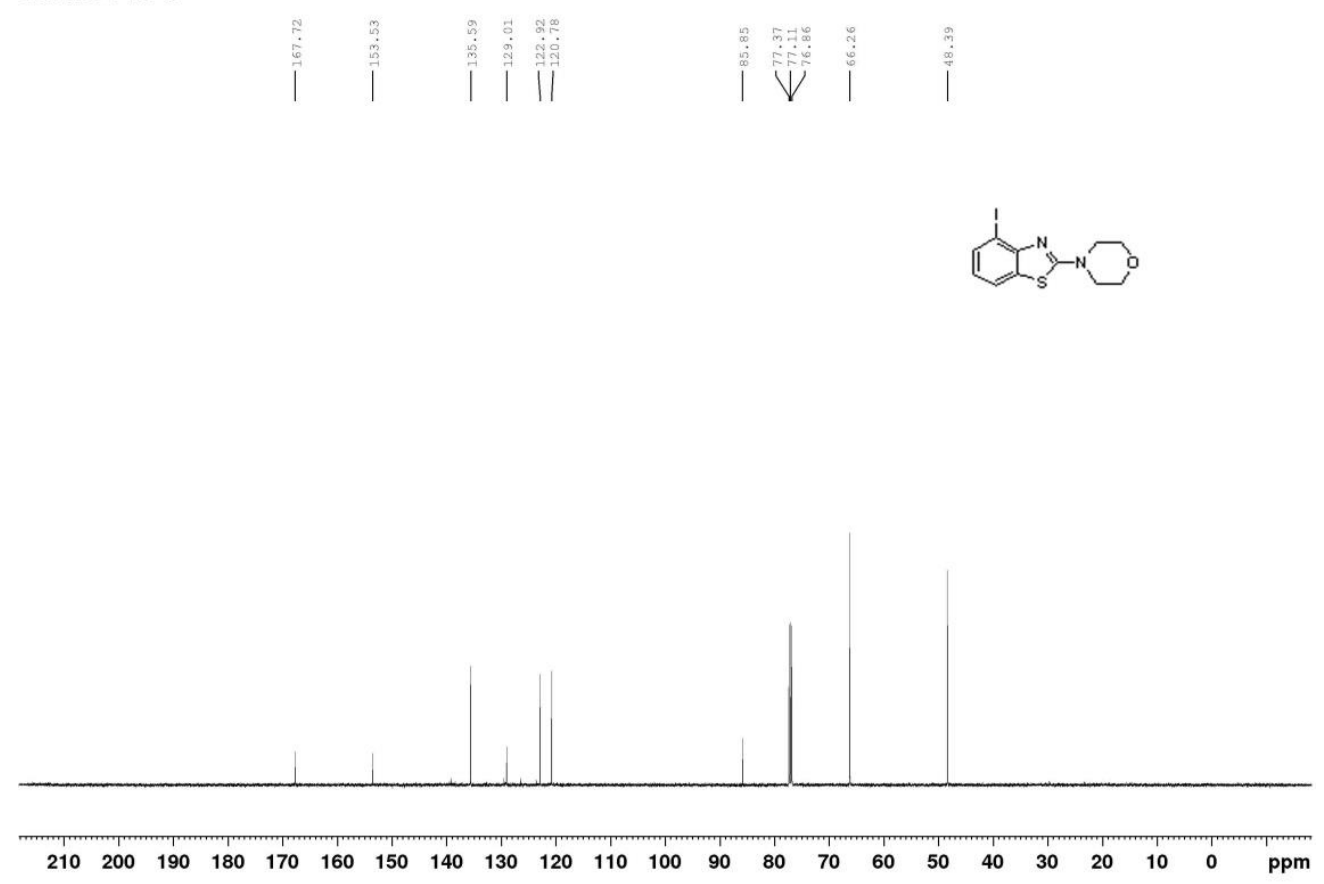

Figure 39: ${ }^{13} \mathrm{C}$ NMR spectra of 4-(4-iodobenzo[d]thiazol-2-yl)morpholine (9p).

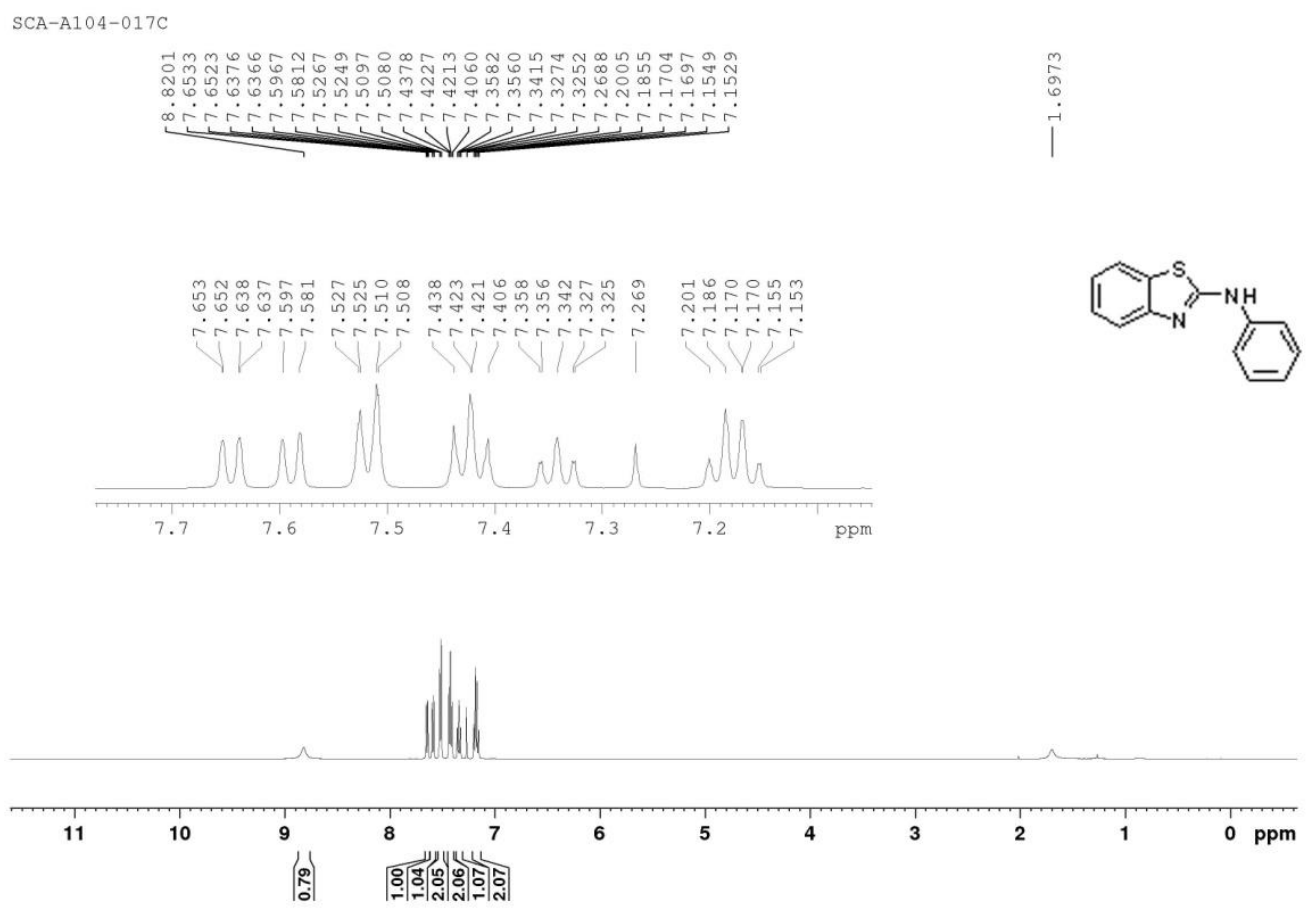

Figure 40: ${ }^{1} \mathrm{H}$ NMR spectra of $N$-phenylbenzo[d]thiazol-2-amine (9q). 


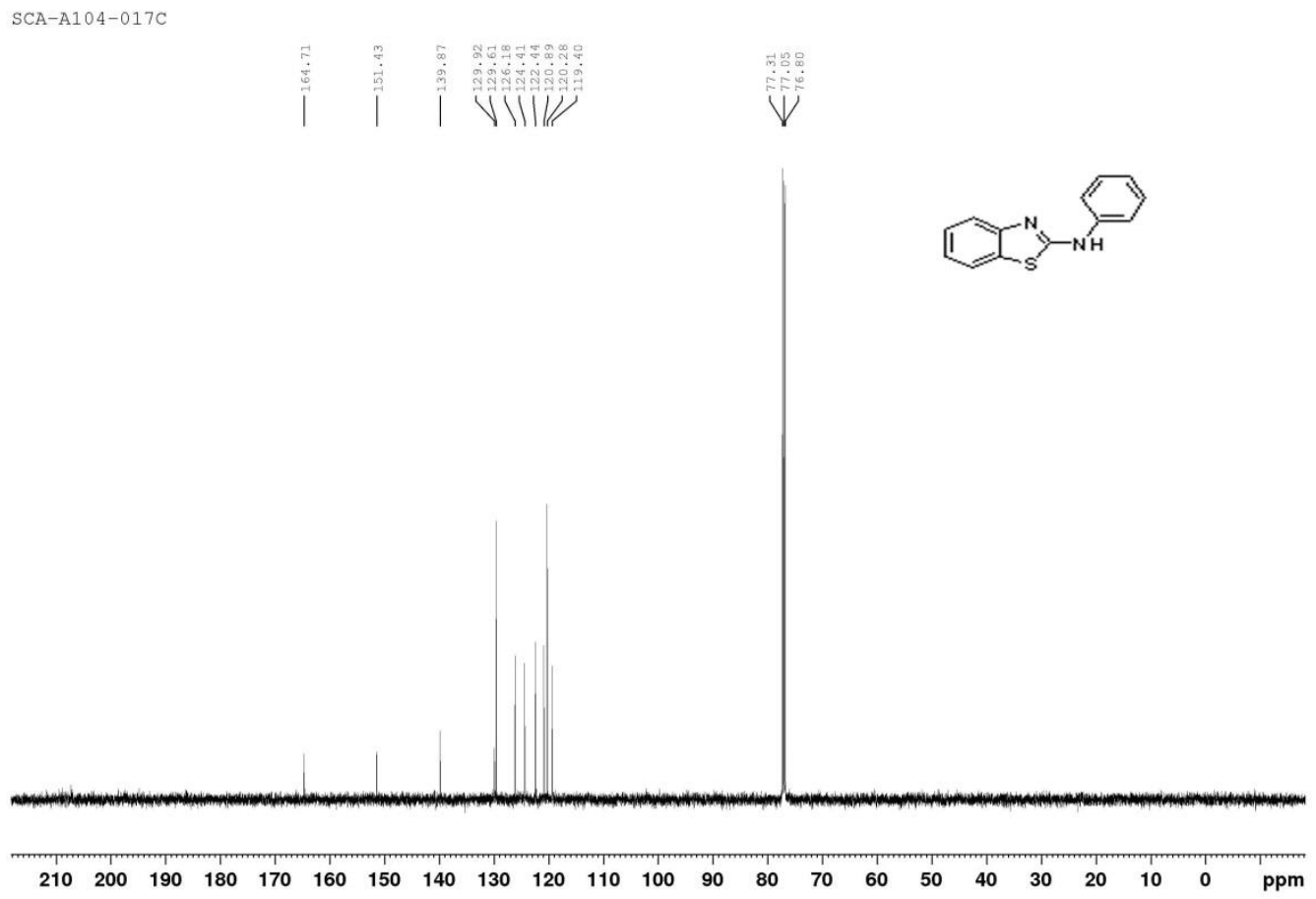

Figure 41: ${ }^{13} \mathrm{C}$ NMR spectra of $N$-phenylbenzo[d]thiazol-2-amine (9q).

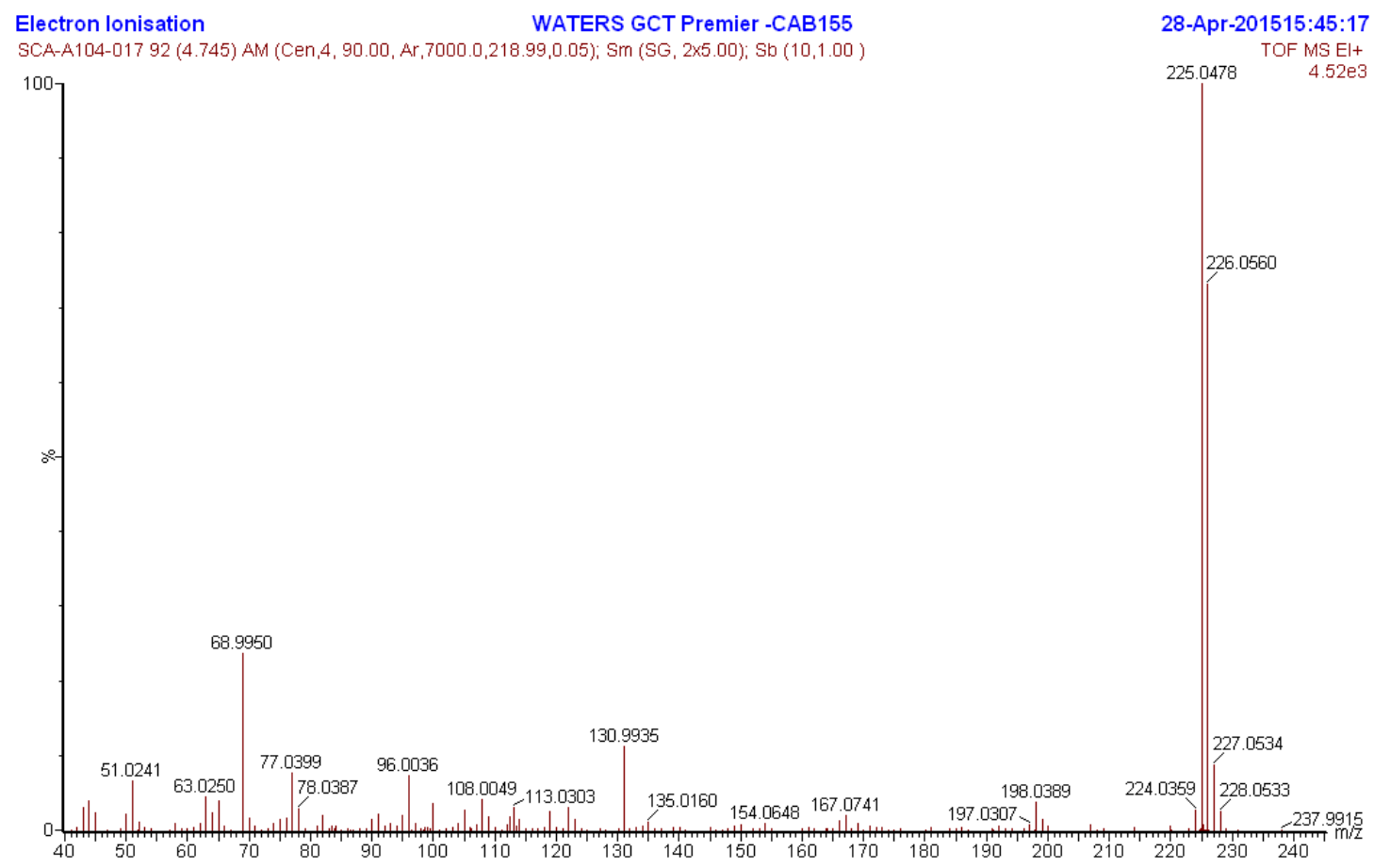

Figure 42: HRMS-EI spectra of $N$-phenylbenzo[d]thiazol-2-amine (9q). 


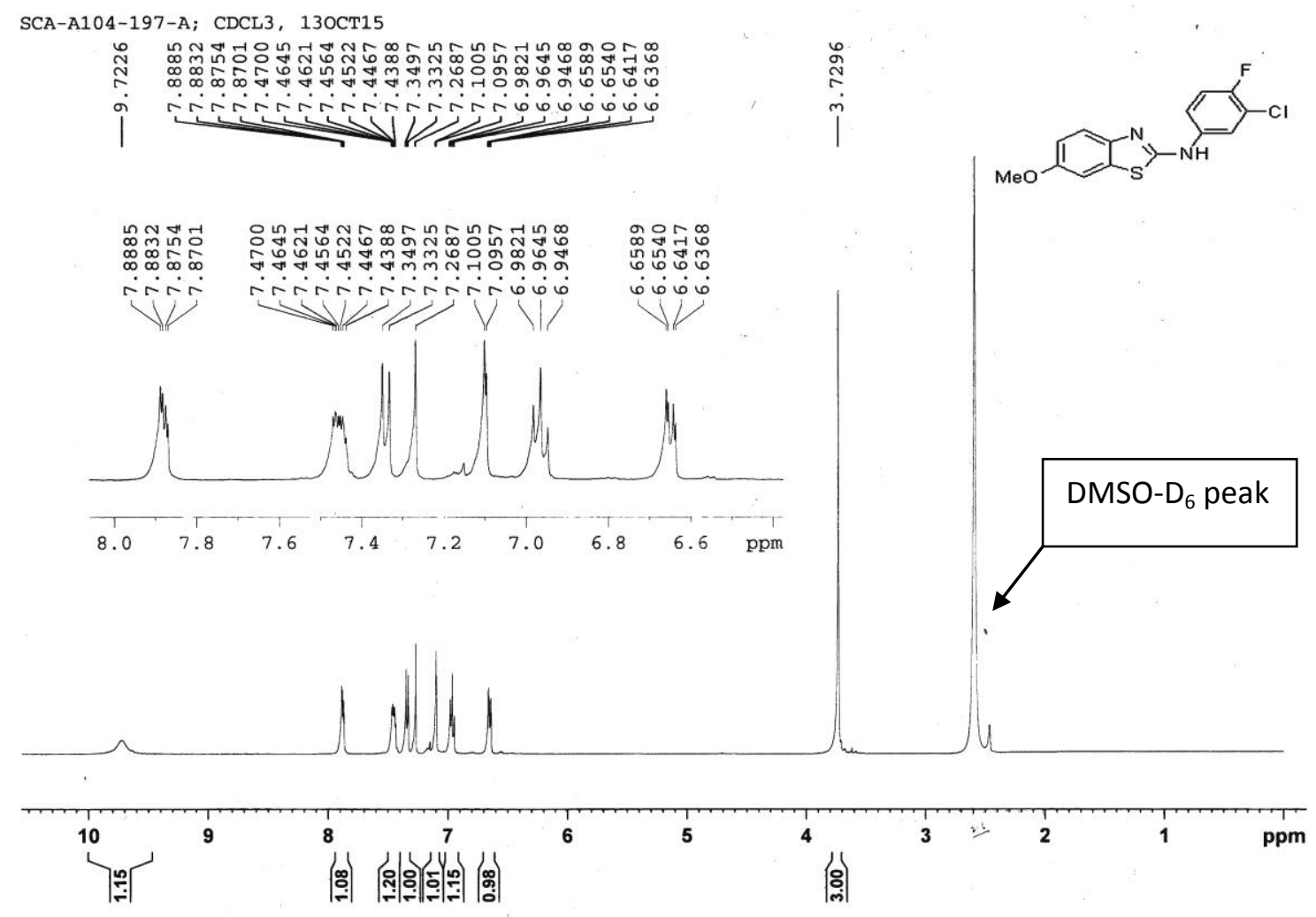

Figure 43: ${ }^{1} \mathrm{H}$ NMR spectra of $N$-(4-chloro-3-fluorophenyl)-5-methoxybenzo[d]thiazol-2-amine (9r).

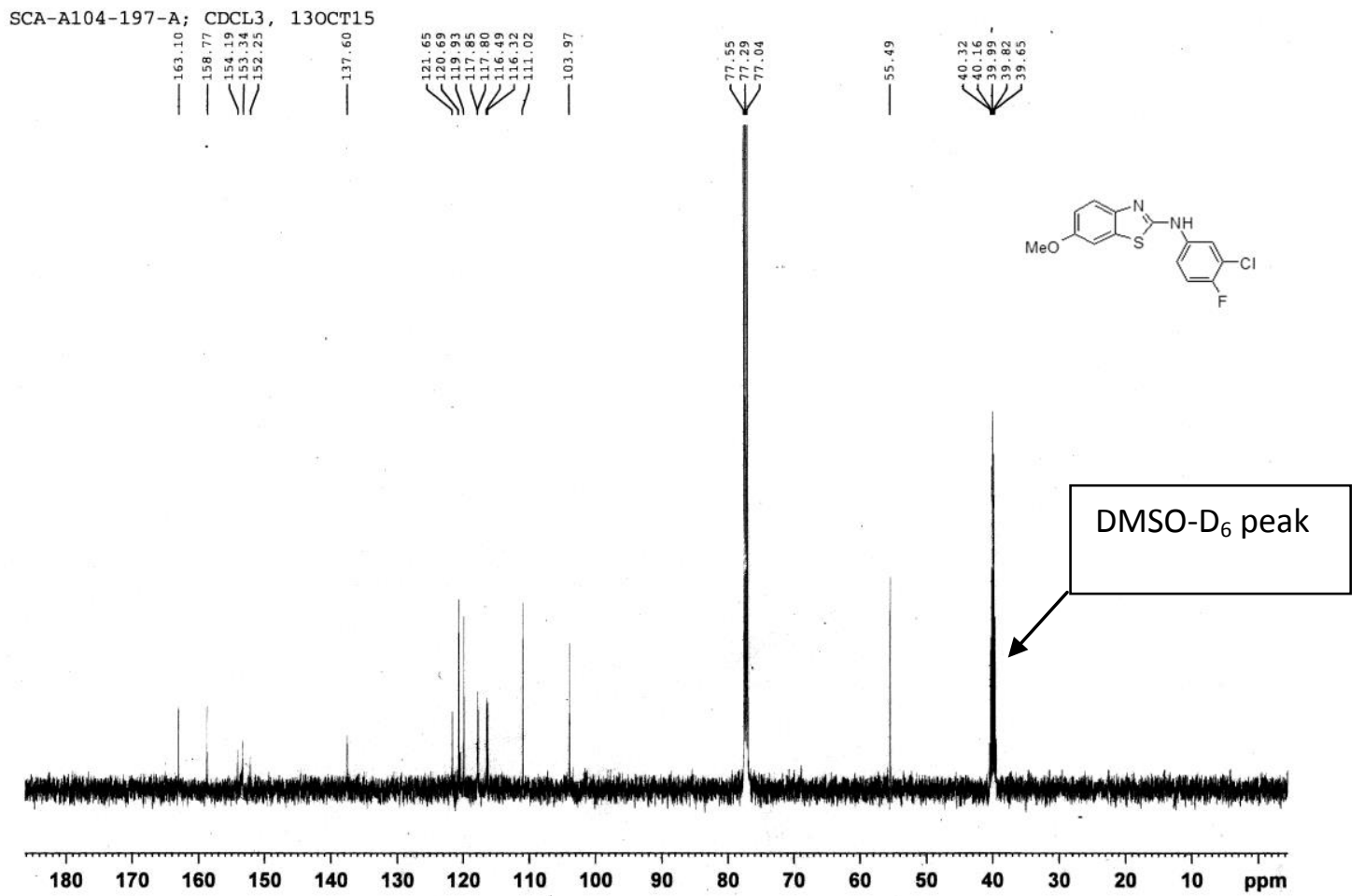

Figure 44: ${ }^{13} \mathrm{C}$ NMR spectra of of $N$-(4-chloro-3-fluorophenyl)-5-methoxybenzo[d]thiazol-2amine (9r). 

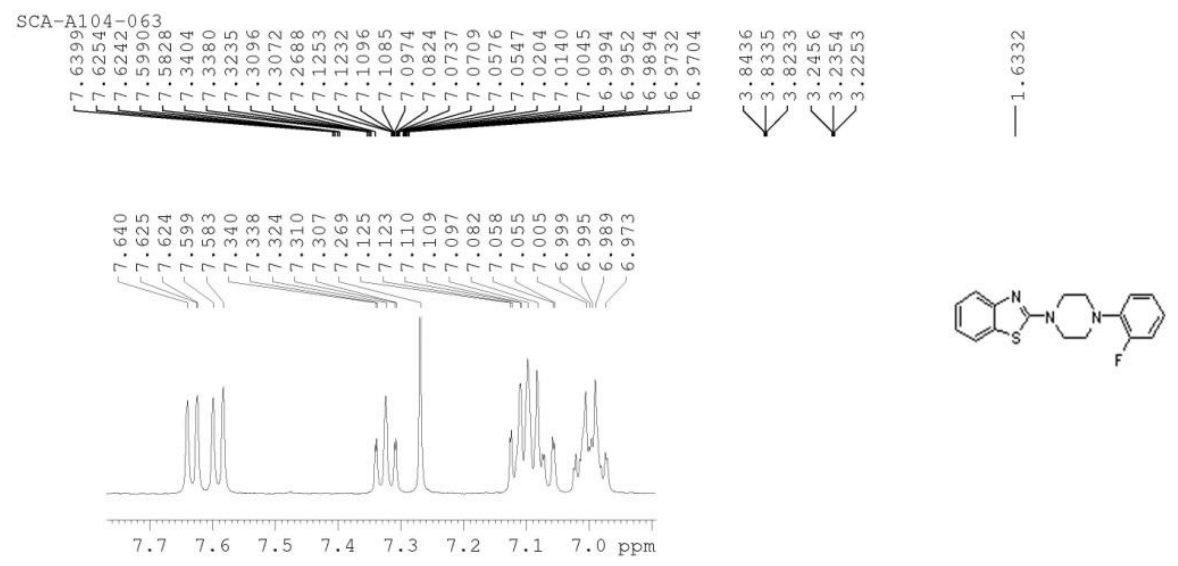

a M M

\begin{tabular}{|c|c|c|c|c|c|c|c|c|c|c|c|}
\hline 10 & 9 & & 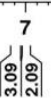 & 6 & 5 & & (ָ) & 2 & 1 & 0 & $\mathrm{ppm}$ \\
\hline
\end{tabular}

Figure S45: ${ }^{1}$ H NMR spectra of 2-(4-(2-fluorophenyl)piperazin-1-yl)benzo[d]thiazole (9s).

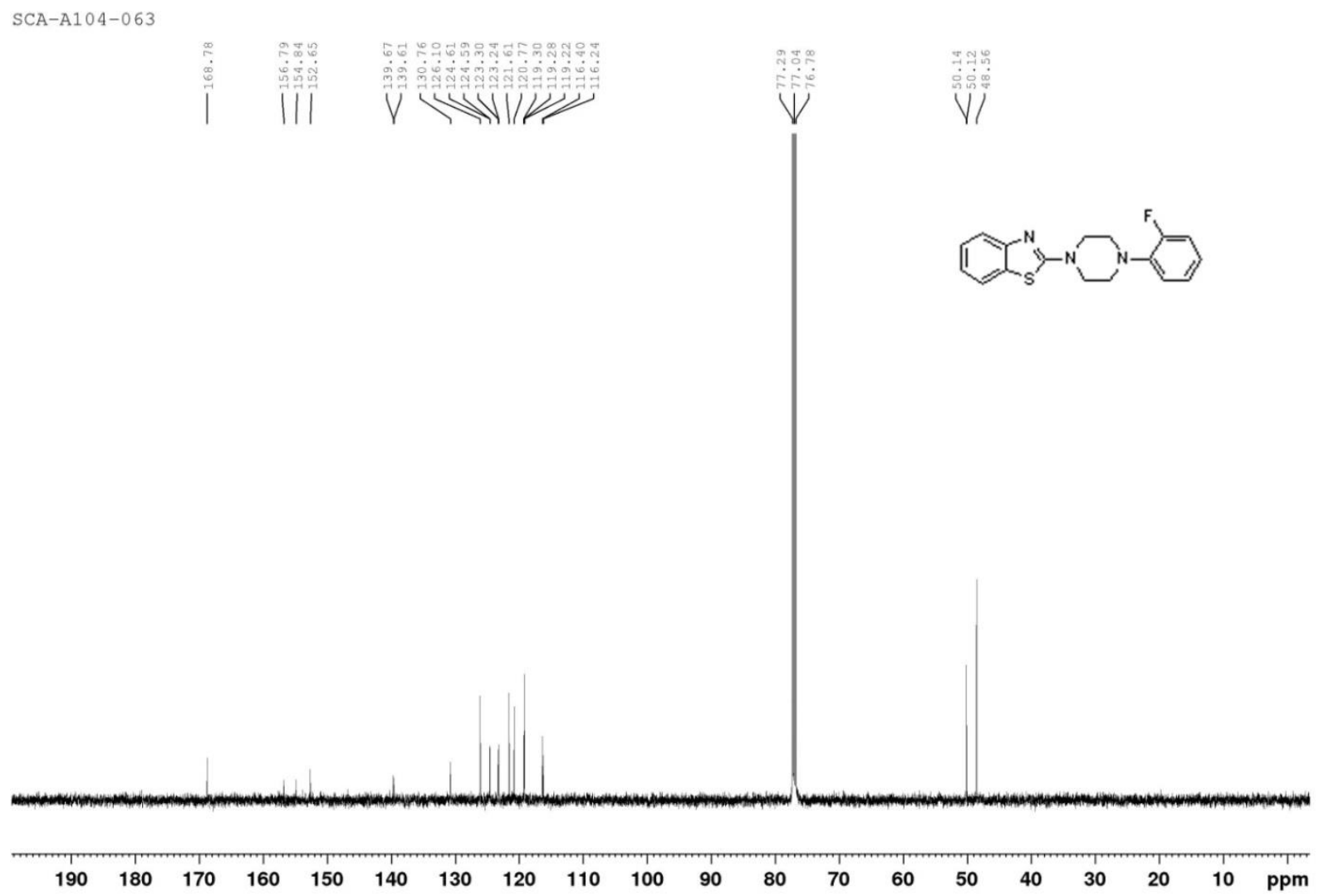

Figure S46: ${ }^{13} \mathrm{C}$ NMR spectra of 2-(4-(2-fluorophenyl)piperazin-1-yl)benzo[d]thiazole (9s). 


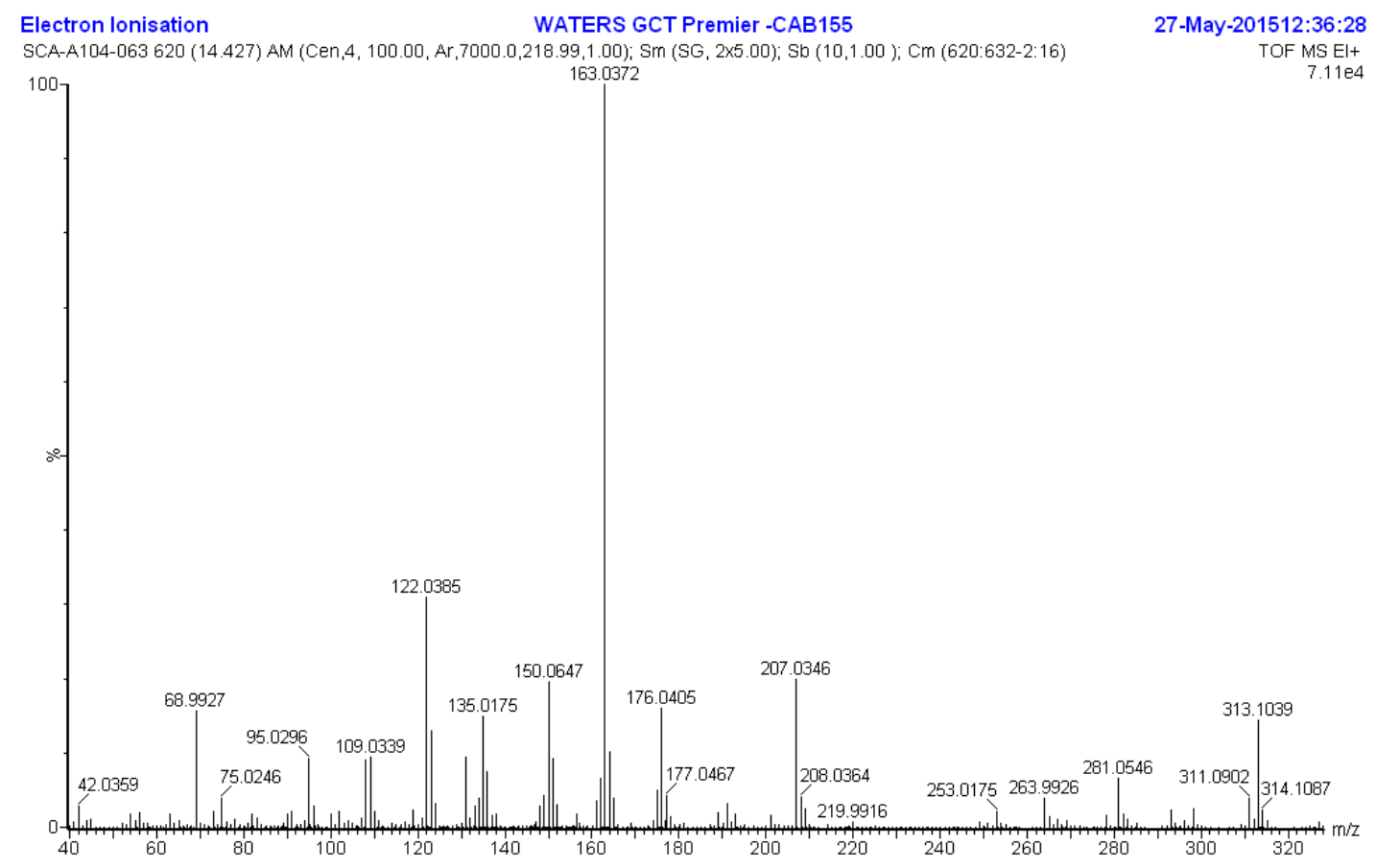

Figure S47: HRMS-EI spectra of 2-(4-(2-fluorophenyl)piperazin-1-yl)benzo[d]thiazole (9s).

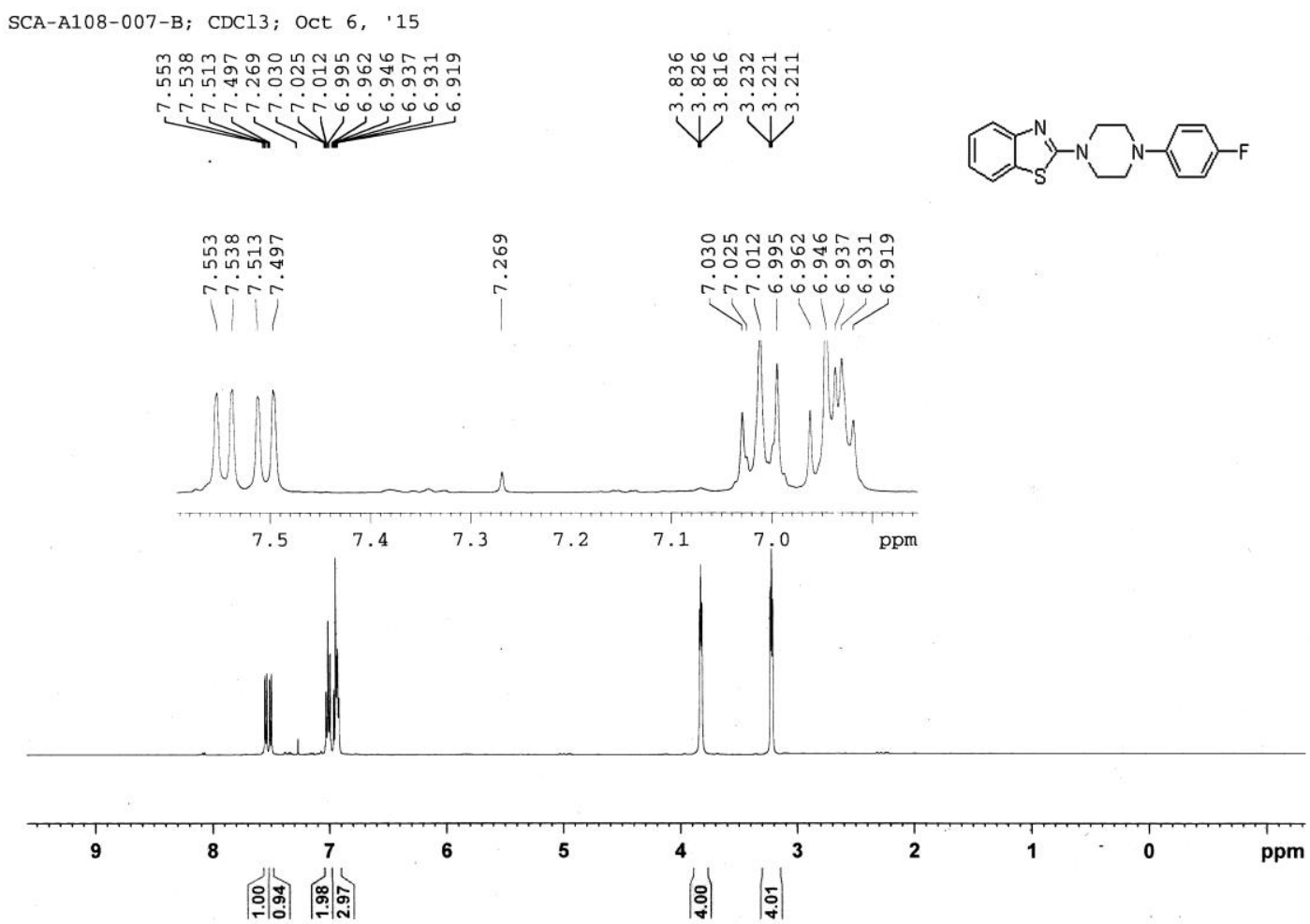

Figure S48: ${ }^{1} \mathrm{H}$ NMR spectra of 2-(4-(4-fluorophenyl)piperazin-1-yl)benzo[d]thiazole (9t). 


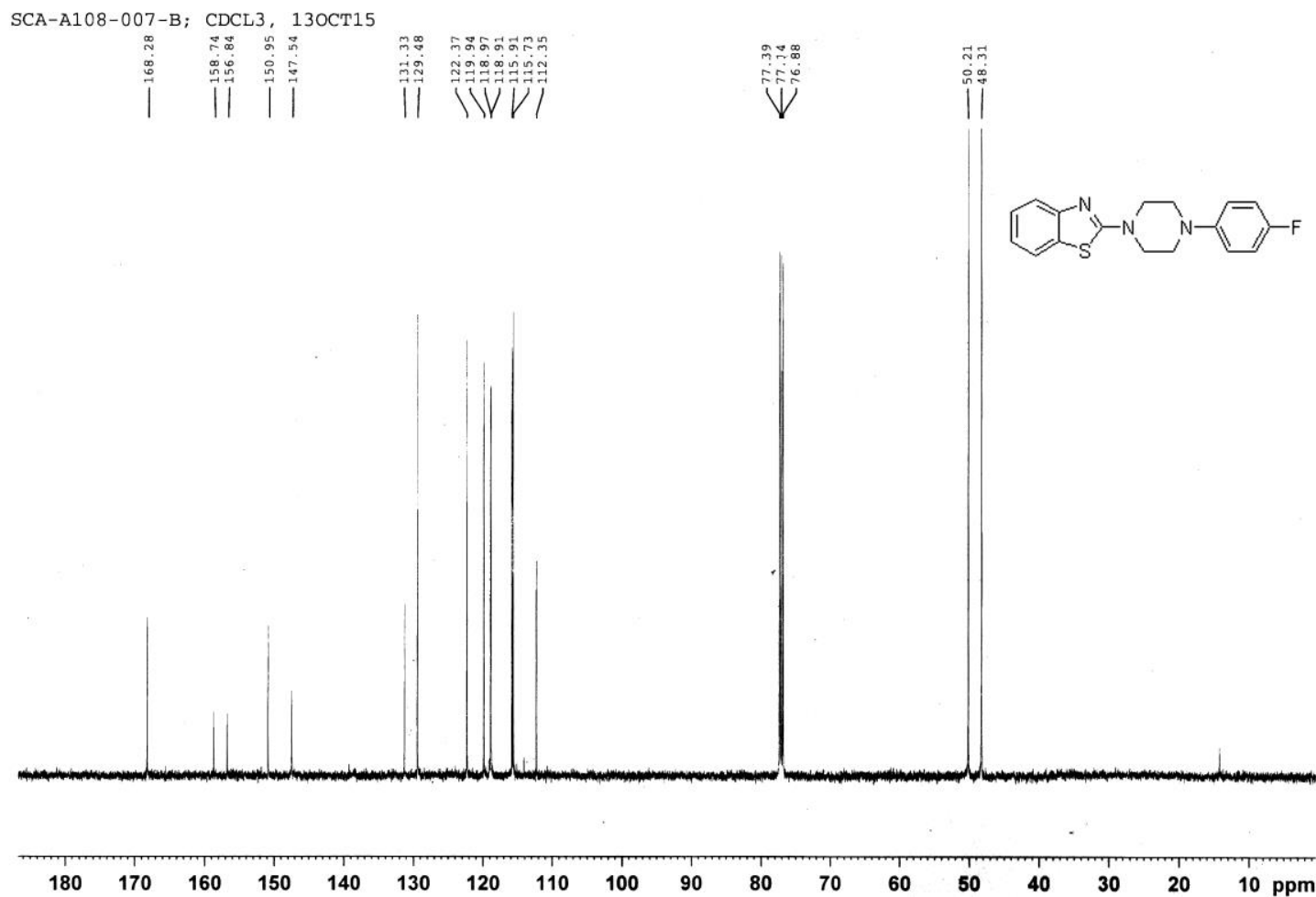

Figure S49: ${ }^{13} \mathrm{C}$ NMR spectra of 2-(4-(4-fluorophenyl)piperazin-1-yl)benzo[d]thiazole (9t).

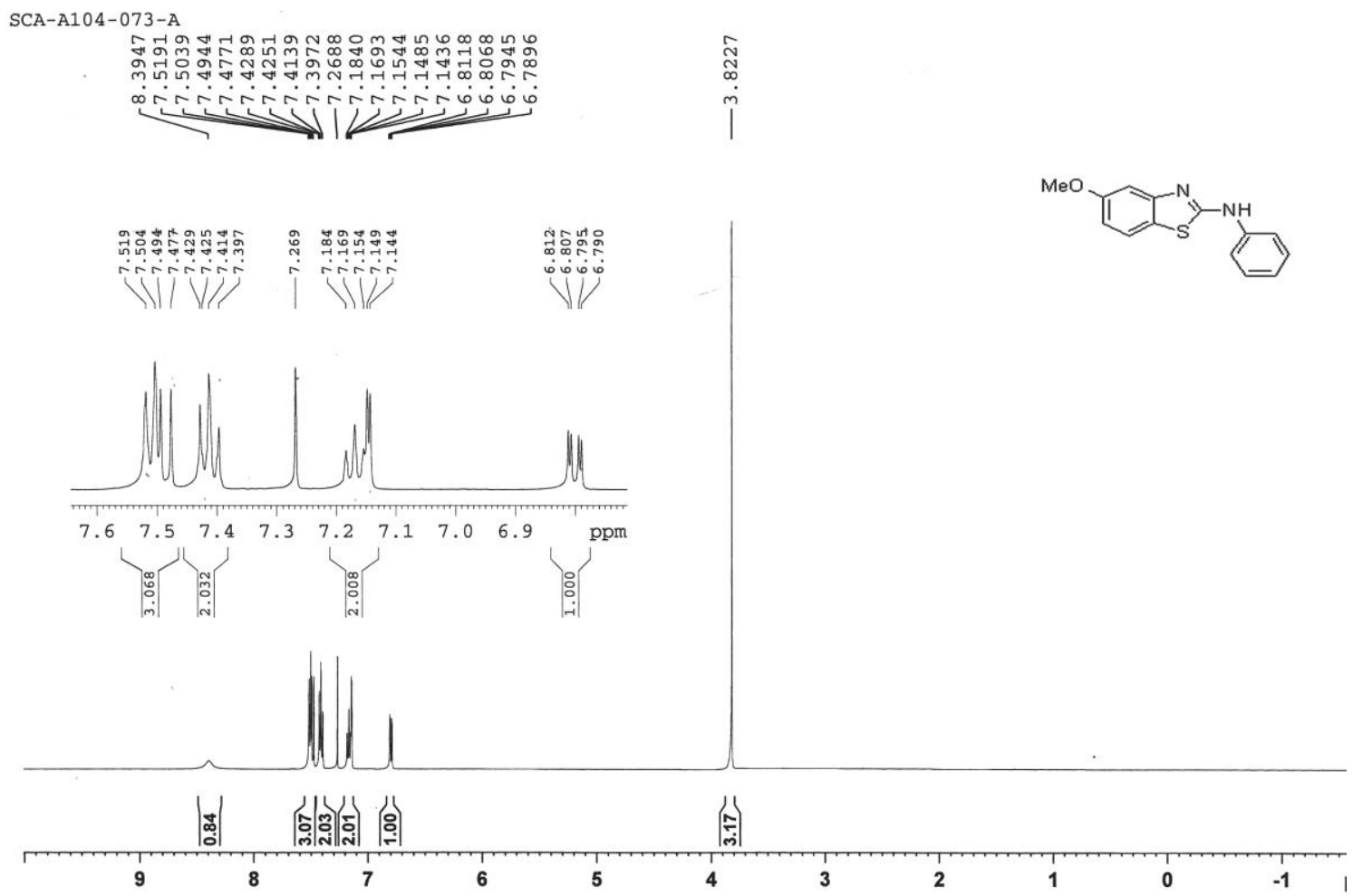

Figure S50: ${ }^{1} \mathrm{H}$ NMR spectra of 5-methoxy-N-phenylbenzo[d]thiazol-2-amine (9u). 

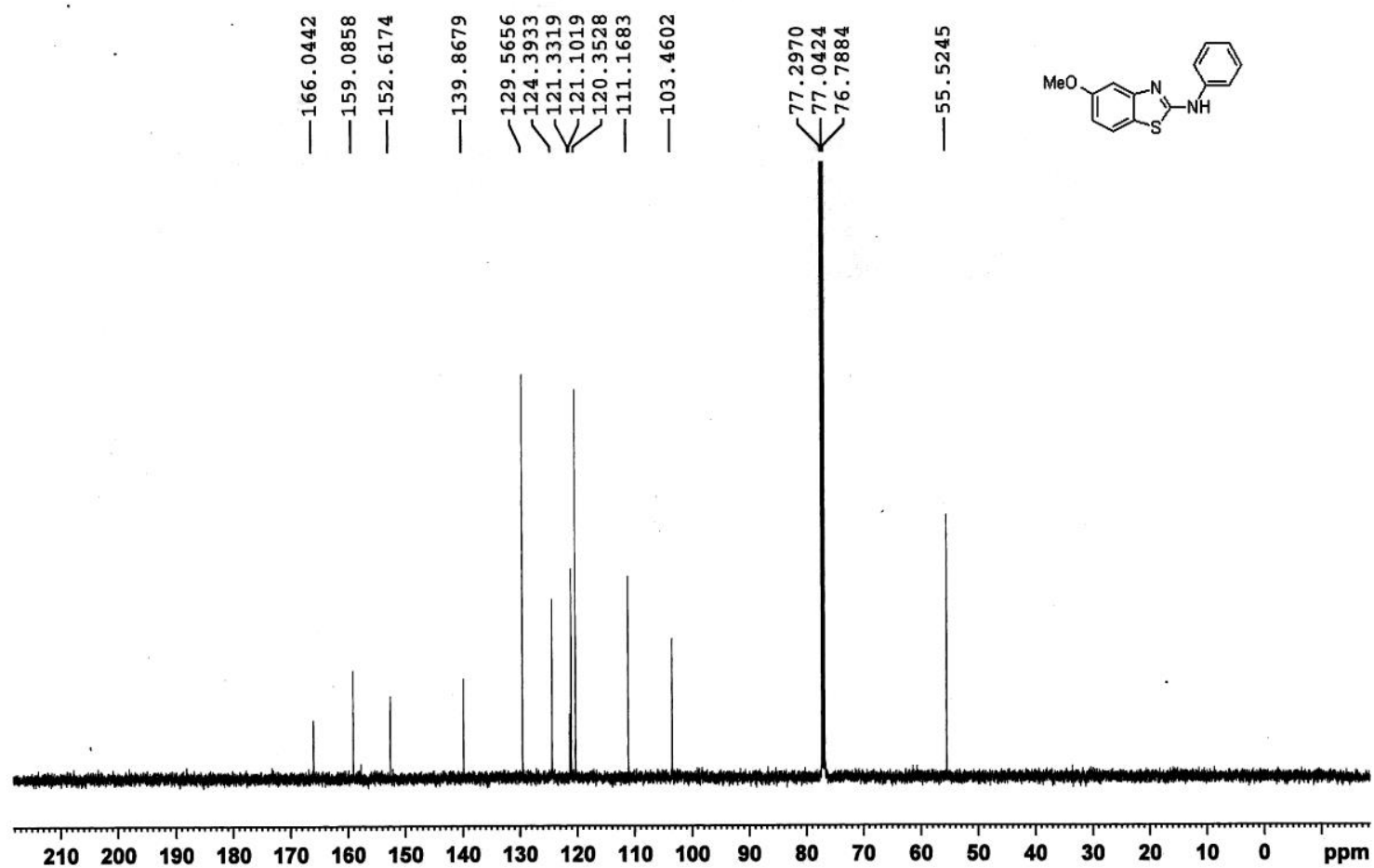

Figure S51: ${ }^{13} \mathrm{C}$ NMR spectra of 5-methoxy-N-phenylbenzo[d]thiazol-2-amine (9u).

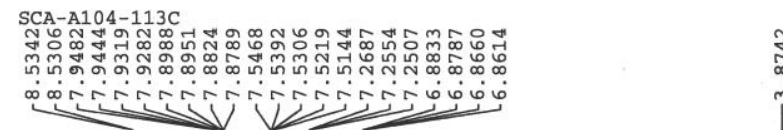

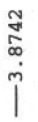
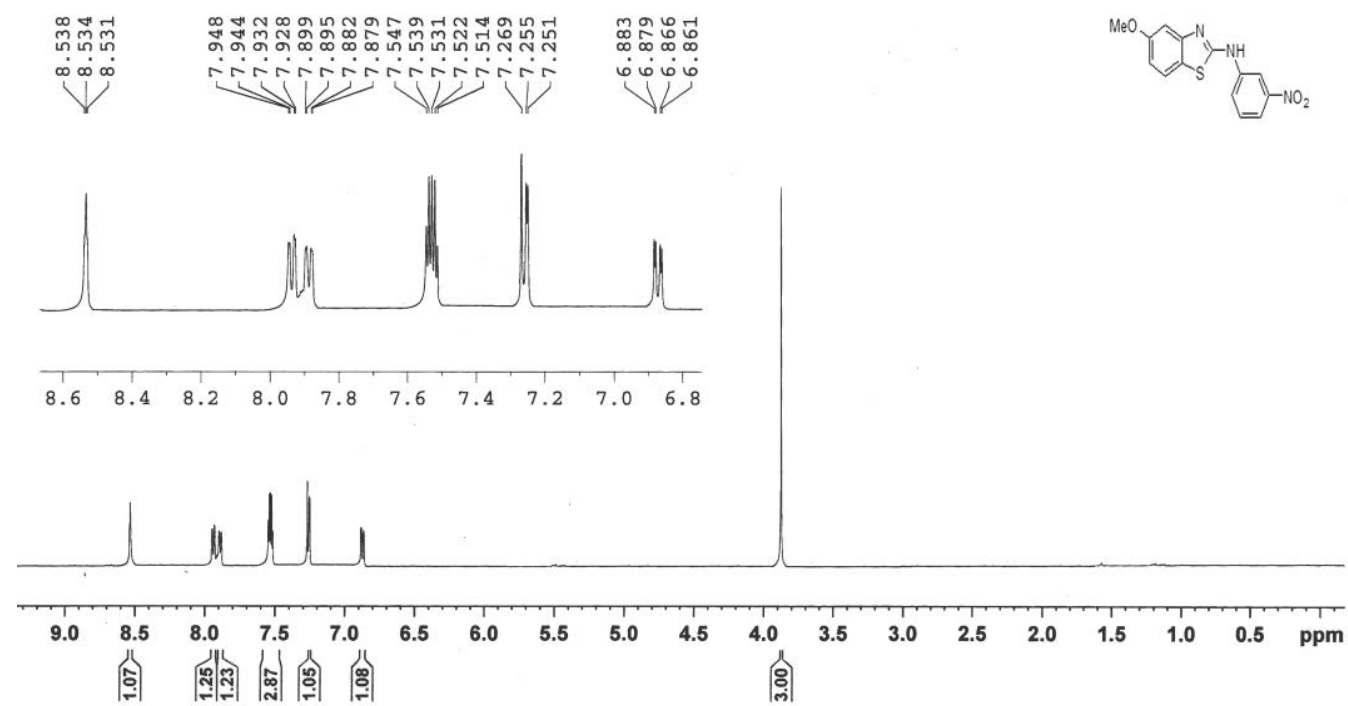

Figure S52: ${ }^{1}$ H NMR spectra of 5-methoxy-N-(3-nitrophenyl)benzo[d]thiazol-2-amine (9v). 


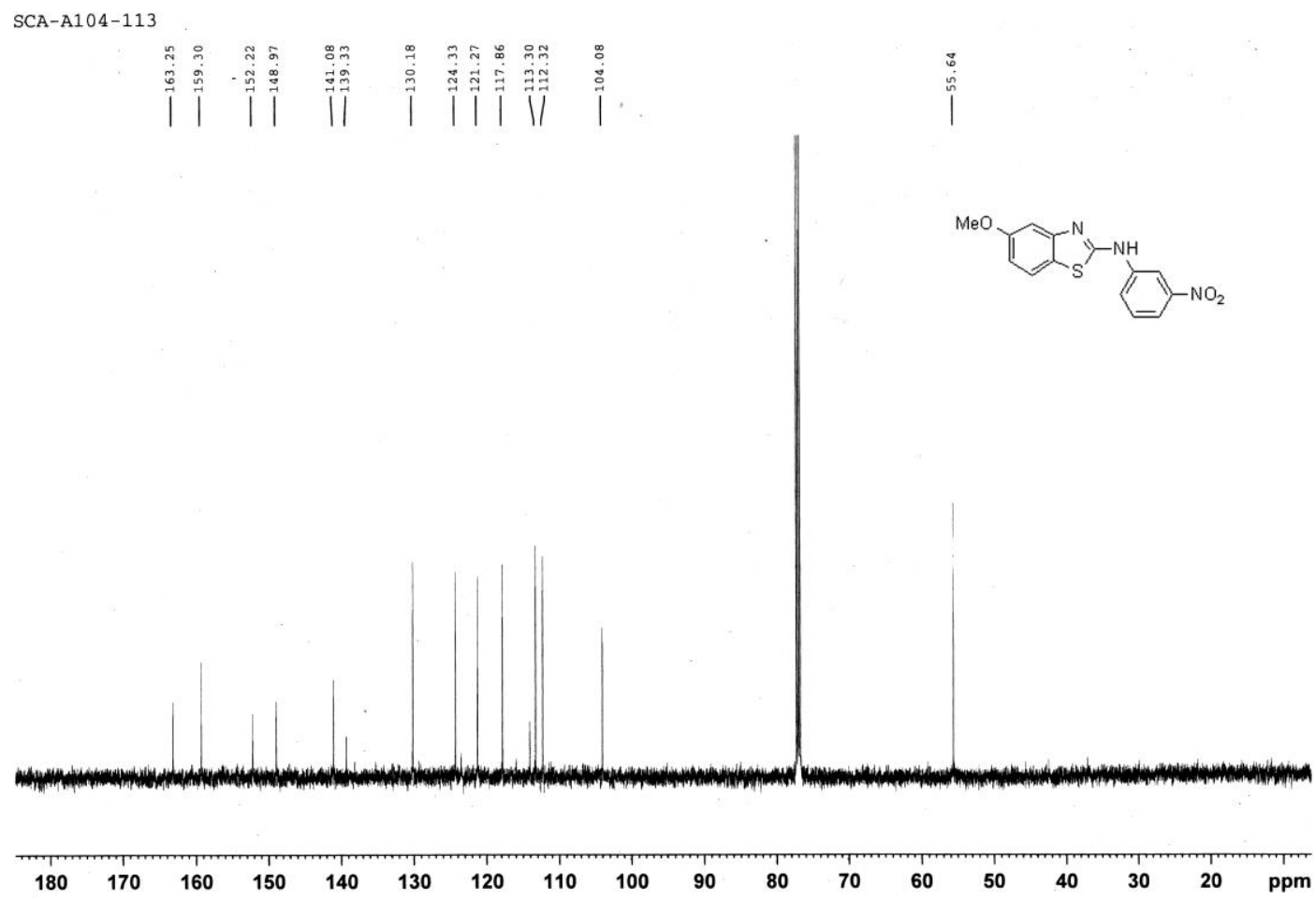

Figure S53: ${ }^{13} \mathrm{C}$ NMR spectra of 5-methoxy-N-(3-nitrophenyl)benzo[d]thiazol-2-amine (9v).

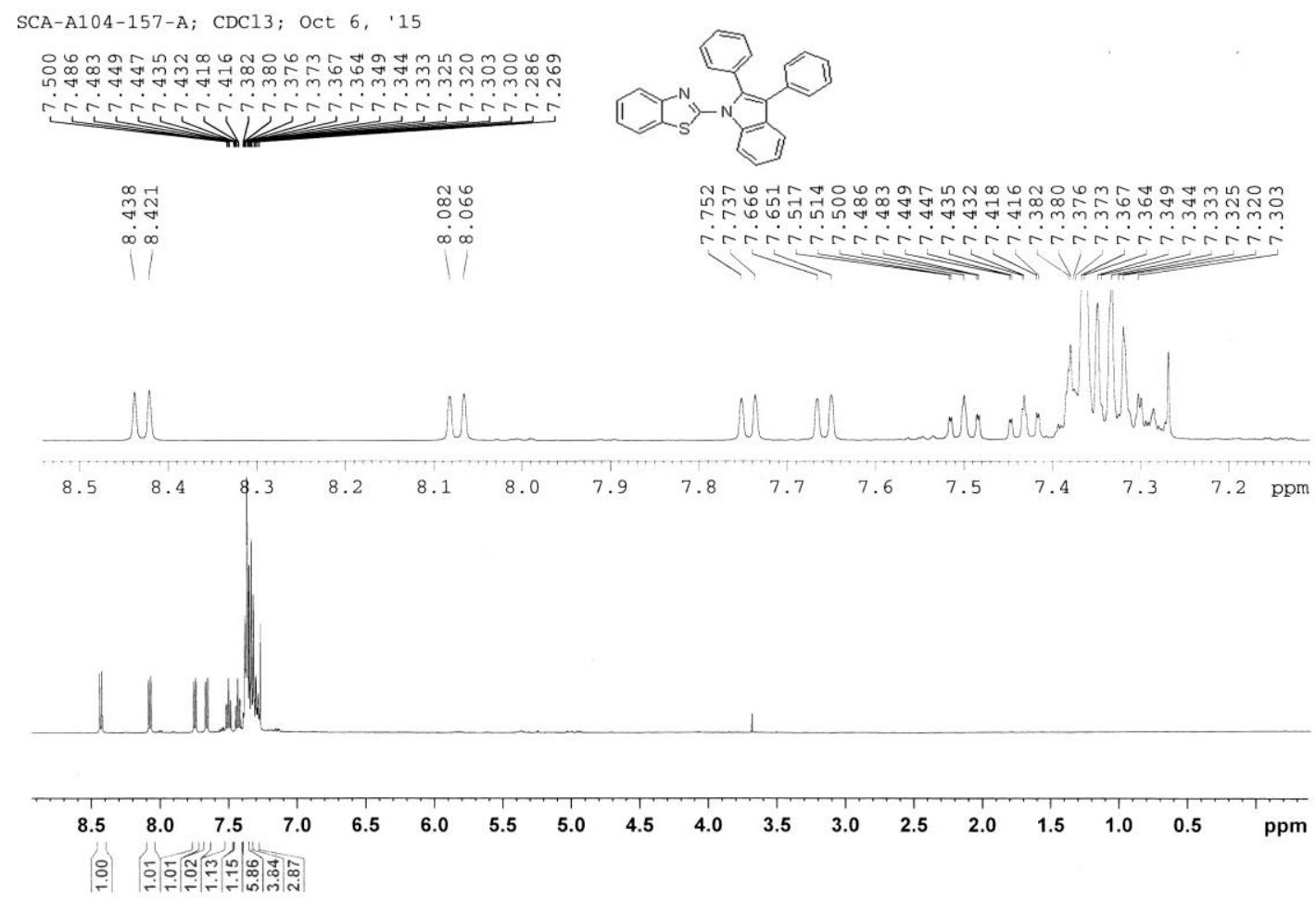

Figure S54: ${ }^{1} \mathrm{H}$ NMR spectra of 2-(2,3-diphenyl-1H-indol-1-yl)benzo[d]thiazole (16). 

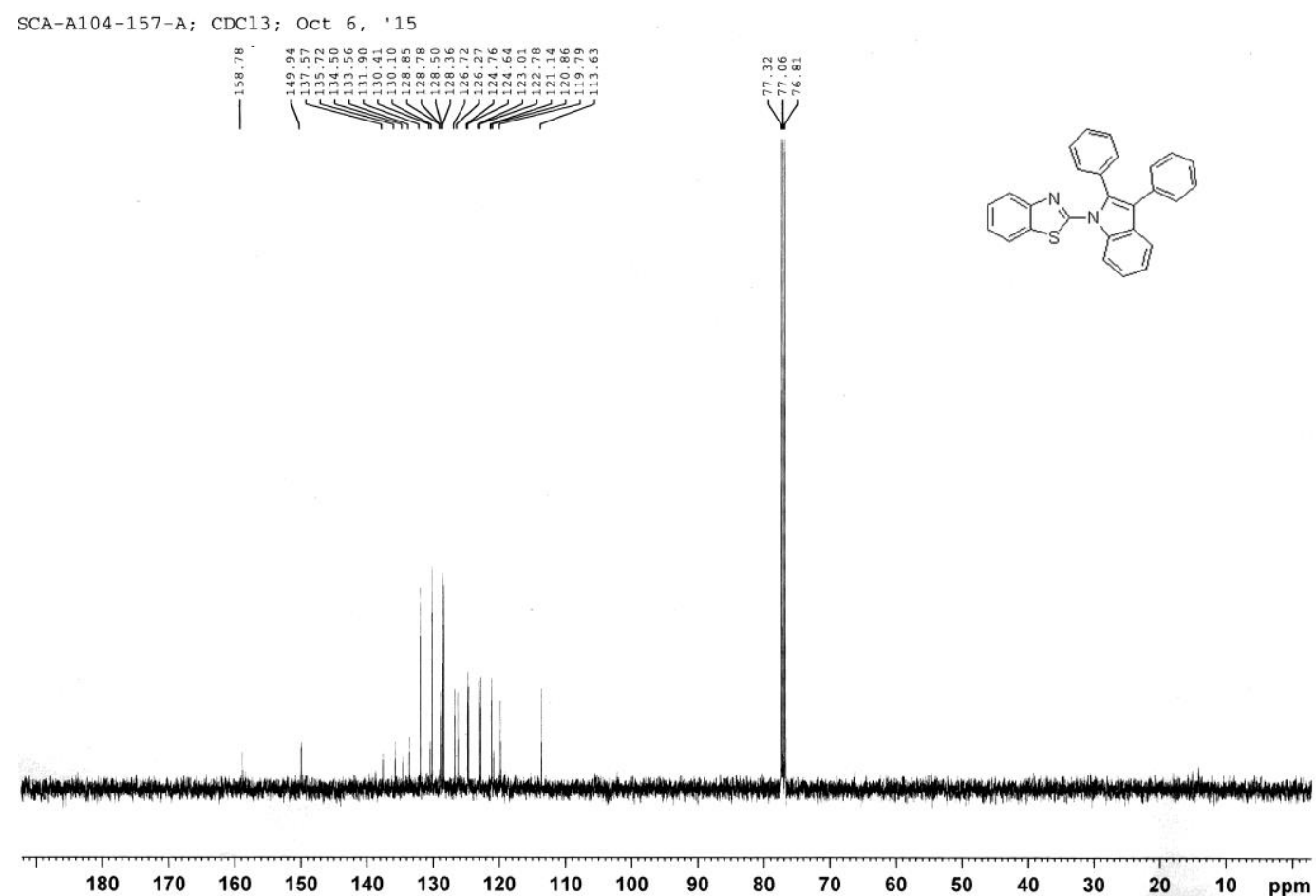

Figure S55: ${ }^{13} \mathrm{C}$ NMR spectra of 2-(2,3-diphenyl-1H-indol-1-yl)benzo[d]thiazole (16). 


\section{Kinetic Isotopic Effect Experiment: Preparation of $N$-2-deutriumphenylmorpholinse-4- carbothioamide}

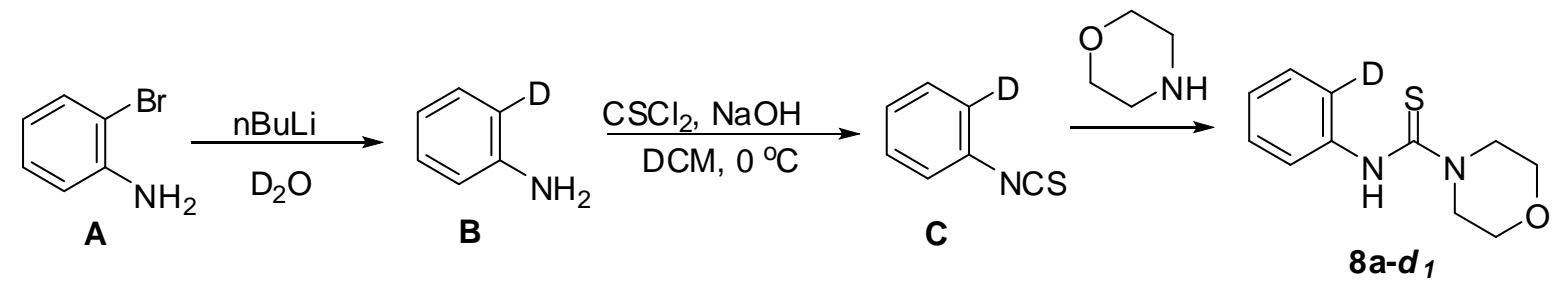

5.1. Preparation of 2-deuterium aniline (B): 2-Bromoaniline (1.5gm) was dissolved in dry diethylether $(9 \mathrm{ml})$ and washed with deuterium oxide $(3 \times 5 \mathrm{ml})$ to replace most of the amine protons by deuterium. The ethereal layer is dried over $\mathrm{Na}_{2} \mathrm{SO}_{4}$, filtered and concentrated to get 1 gm $(5.81 \mathrm{mmol})$ of yellow liquid, which was dissolved in $5 \mathrm{~mL}$ of anhydrous THF and transferred to the reaction flask via a syringe, the flask was evacuated and replaced by argon (thrice) and then ${ }^{n} \mathrm{BuLi}(18.4 \mathrm{~mL}, 29 \mathrm{mmol}, 1.6 \mathrm{M})$ was added to the mixture at $-78{ }^{\circ} \mathrm{C}$. After stirring at $-78^{\circ} \mathrm{C}$ for $1 \mathrm{~h}$, the mixture was warmed to rt and stirred for $1 \mathrm{~h}$. Then, $\mathrm{D}_{2} \mathrm{O}(0.29 \mathrm{~mol})$ was added to the reaction mixture at $-78{ }^{\circ} \mathrm{C}$, stirred for $30 \mathrm{~min}$, warmed to rt and kept at $\mathrm{rt}$ for 1 h. Finally the reaction mixture was quenched with water and extracted with ethyl acetate (100 $\mathrm{mL} \times 2$ ). The organic layers were combined, dried over $\mathrm{Na}_{2} \mathrm{SO}_{4}$ and concentrated in vacuo, and purified by silica gel chromatography (EtOAc: petroleum ether $=20: 80)$ to obtain the desired compound 6; yield: $0.50 \mathrm{~g}(91.4 \%)$. The product so obtained was used in the subsequent reaction.

5.2. Preparationof2-deutriumphenylisothiocyanate $(\mathbf{C})$ : A schlenk tube equipped with a stirbar was charged with pure compound $6(0.5 \mathrm{~g}, 5.31 \mathrm{mmol})$ and dissolved it in $15 \mathrm{~mL}$ DCM. The reaction tube was purged with argon. Then the reaction mixture was cooled to $0{ }^{\circ} \mathrm{C}$ and added thiophosgene $(1.4 \mathrm{~mL}, 14.97 \mathrm{mmol})$ via syringe dropwise. Subsequently, crushed $\mathrm{NaOH}$ was added and the reaction mixture was stirred for $1 \mathrm{hr}$. On completion of reaction on TLC, the reaction mixture was extracted with DCM $(20 \mathrm{~mL} \times 2)$. The organic layers were combined, dried over $\mathrm{Na}_{2} \mathrm{SO}_{4}$, and concentrated under reduced pressure to obtain the crude 7 .

5.3. Preparation of $\mathbf{N}$-2-deutriumphenylmorpholine-4-carbothioamide 8a- $\boldsymbol{d}_{\boldsymbol{l}}$ : A $10 \mathrm{~mL}$ round bottom flask was charged with crude $7(0.2 \mathrm{gm}, 1.468 \mathrm{mmol})$ and morpholine $(0.116 \mathrm{gm}$, $1.334 \mathrm{mmol}$ ) under neat reactions at $\mathrm{rt}$. The reaction stirred for $1 \mathrm{~h}$ to obtained gummy solid. The reaction mixture was purified by silica gel chromatography (EtOAc: petroleum ether $=20: 80$ ) to obtain the desired compound $\mathbf{8 a}-\boldsymbol{d}_{\boldsymbol{l}}$. Product: white solid, yield: $0.380 \mathrm{~g}(75 \%, 95 \%$ deuterium incorporation); m.p.: $118.5{ }^{\circ} \mathrm{C} .{ }^{1} \mathrm{H}$ NMR $(\delta \mathrm{ppm}):\left(500 \mathrm{MHz}, \mathrm{CDCl}_{3}\right), 7.35(\mathrm{~m}, 2 \mathrm{H}$, aromatic C$H, J=\mathrm{Hz}), 7.31(\mathrm{bsr}, 1 \mathrm{H}, \mathrm{N}-H), 7.17(\mathrm{t}, 1 \mathrm{H}$, aromatic C- $H, J=7.5 \mathrm{~Hz}), 7.13(\mathrm{~d}, 1 \mathrm{H}$, aromatic C$H, J=8 \mathrm{~Hz}), 3.82\left(\mathrm{t}, 4 \mathrm{H}, \mathrm{sp}^{3} \mathrm{C}-H, J=4.8 \mathrm{~Hz}\right), 3.73\left(\mathrm{t}, 4 \mathrm{H}, \mathrm{sp}^{3} \mathrm{C}-H, J=4.82 \mathrm{~Hz}\right) .{ }^{13} \mathrm{C}$ NMR $(\delta$ ppm): (125 MHz, $\left.\mathrm{CDCl}_{3}\right): 183.81,139.74,129.30,129.20,125.42,122.92,66.14,49.75$. 


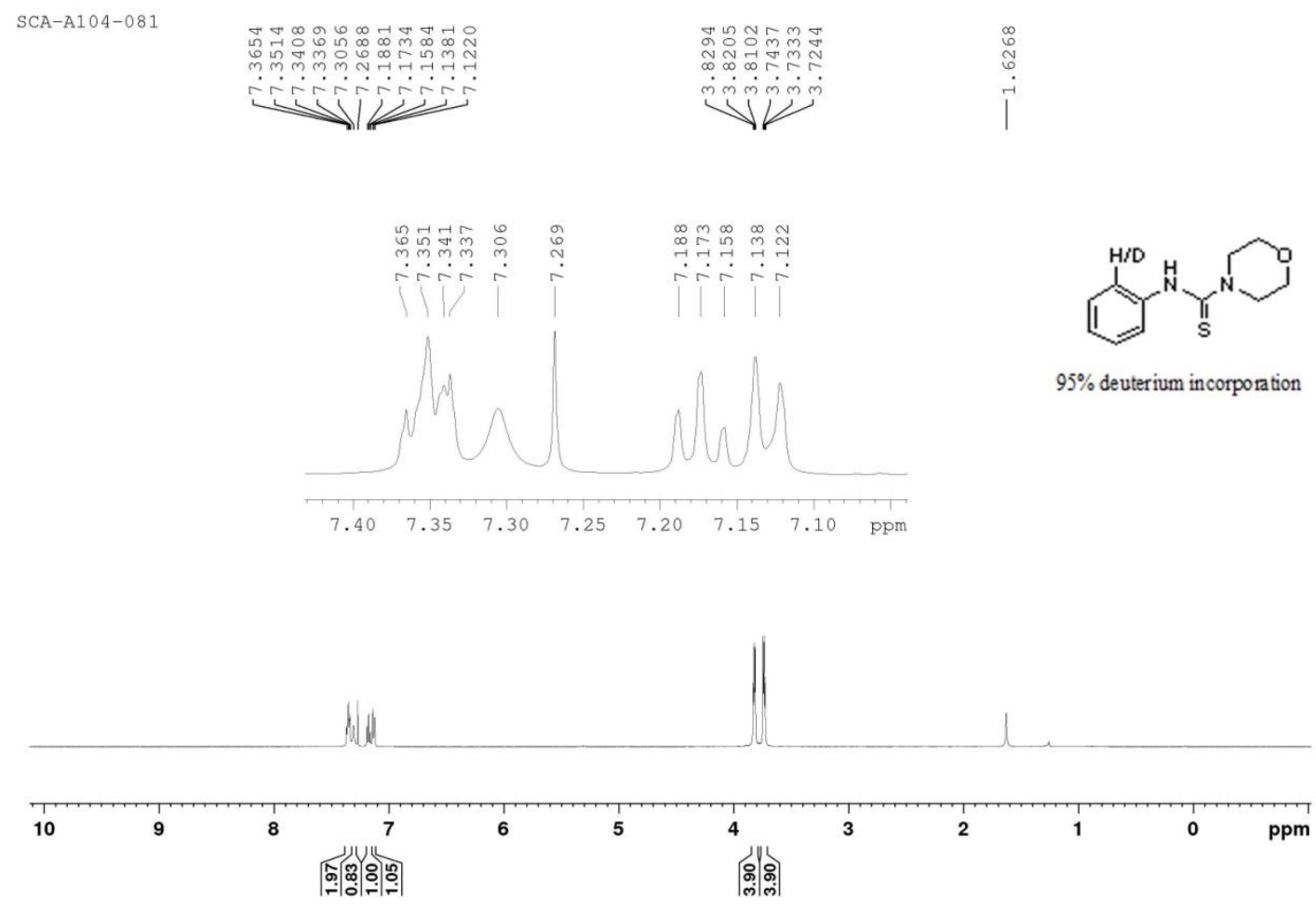

Figure S56: ${ }^{1} \mathrm{H}$ NMR spectra of $N$-2-deutriumphenylmorpholine-4-carbothioamide $\left(\mathbf{8 a}-\boldsymbol{d}_{1}\right)$.
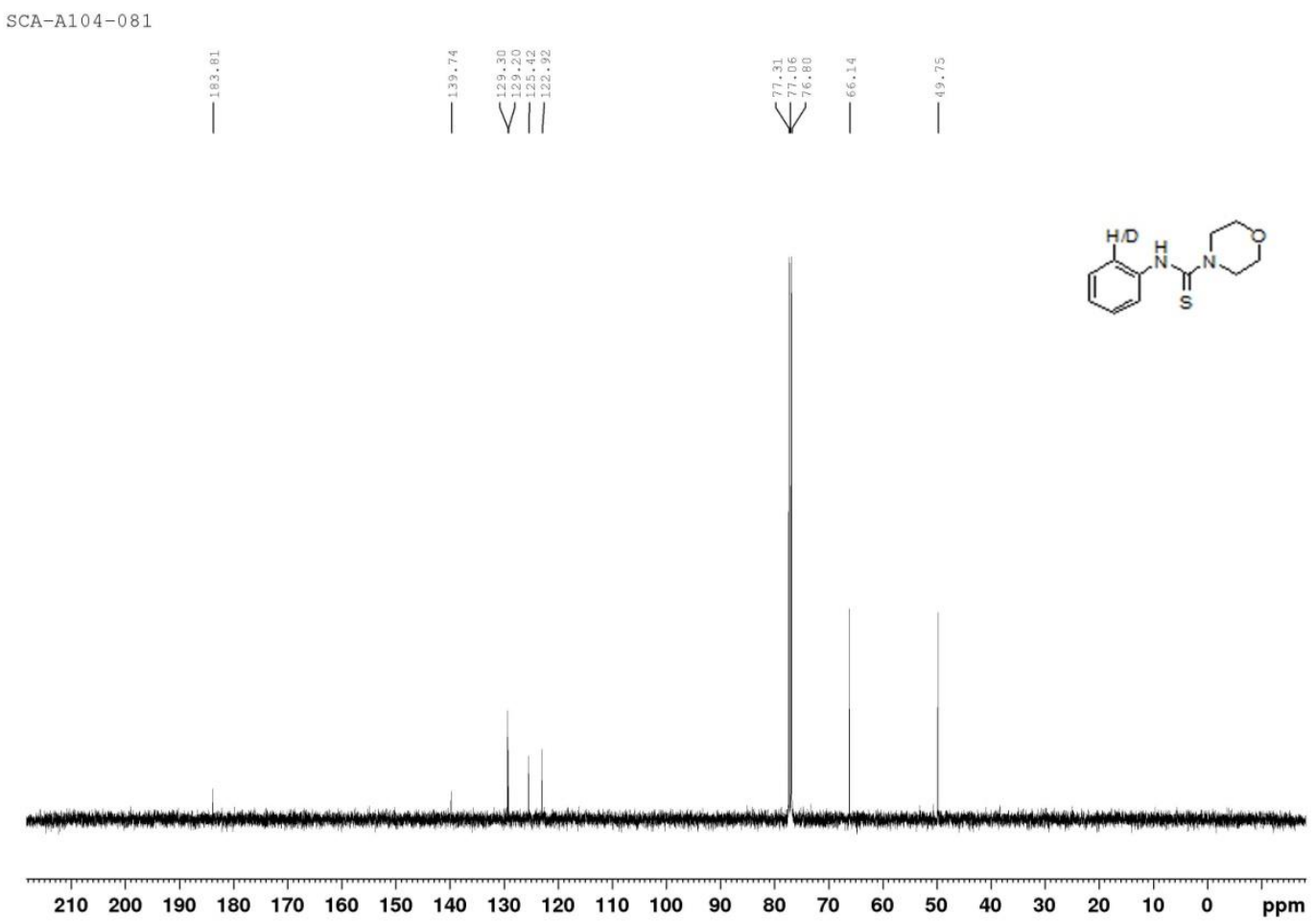

Figure S57: ${ }^{13} \mathrm{C}$ NMR spectra of $N$-2-deutriumphenylmorpholine-4-carbothioamide $\left(\mathbf{8 a}-\boldsymbol{d}_{1}\right)$. 


\subsection{Determination of Kinetic Isotope Effect (KIE) in 2-aminobenzothiazole experiment}

A schlenk tube equipped with a stir-bar was charged with $N$-(2-dueteriopenyl)morpholine-4carbothioamide 8a- $\boldsymbol{d}_{1}(0.05 \mathrm{~g}, 0.223 \mathrm{mmol})$ and DCE $(2 \mathrm{~mL})$ as a solvent. The reaction tube was purged with argon. Then after 5-10 min Oxone $(0.068 \mathrm{~g}, 0.223 \mathrm{mmol})$ and $\mathrm{RuCl}_{3}(0.002 \mathrm{~g}, 0.011$ $\mathrm{mmol}$ ) was added to the reaction mixture followed by argon purging, then argon was replaced by air and the mixture was stirred at $110{ }^{\circ} \mathrm{C}$ for $4 \mathrm{hrs}$. After cooling to room temperature, the reaction mixture was passed through Celite bed and concentrated under reduced pressure and then purified by silica gel chromatography (EtOAc: petroleum ether $=1: 9$ ) to give inseparable mixture of 9a- $d$ and 9a. The ratio of $9 \mathbf{a}-d_{1}$ and $9 \mathbf{a}$ was determined by ${ }^{1} \mathrm{H}$ NMR spectrum as 9a$d_{1}: 9 \mathbf{a}=45:(50+5)\left(\mathrm{KIE}=45 /(55-5)_{-}=0.9\right)$.
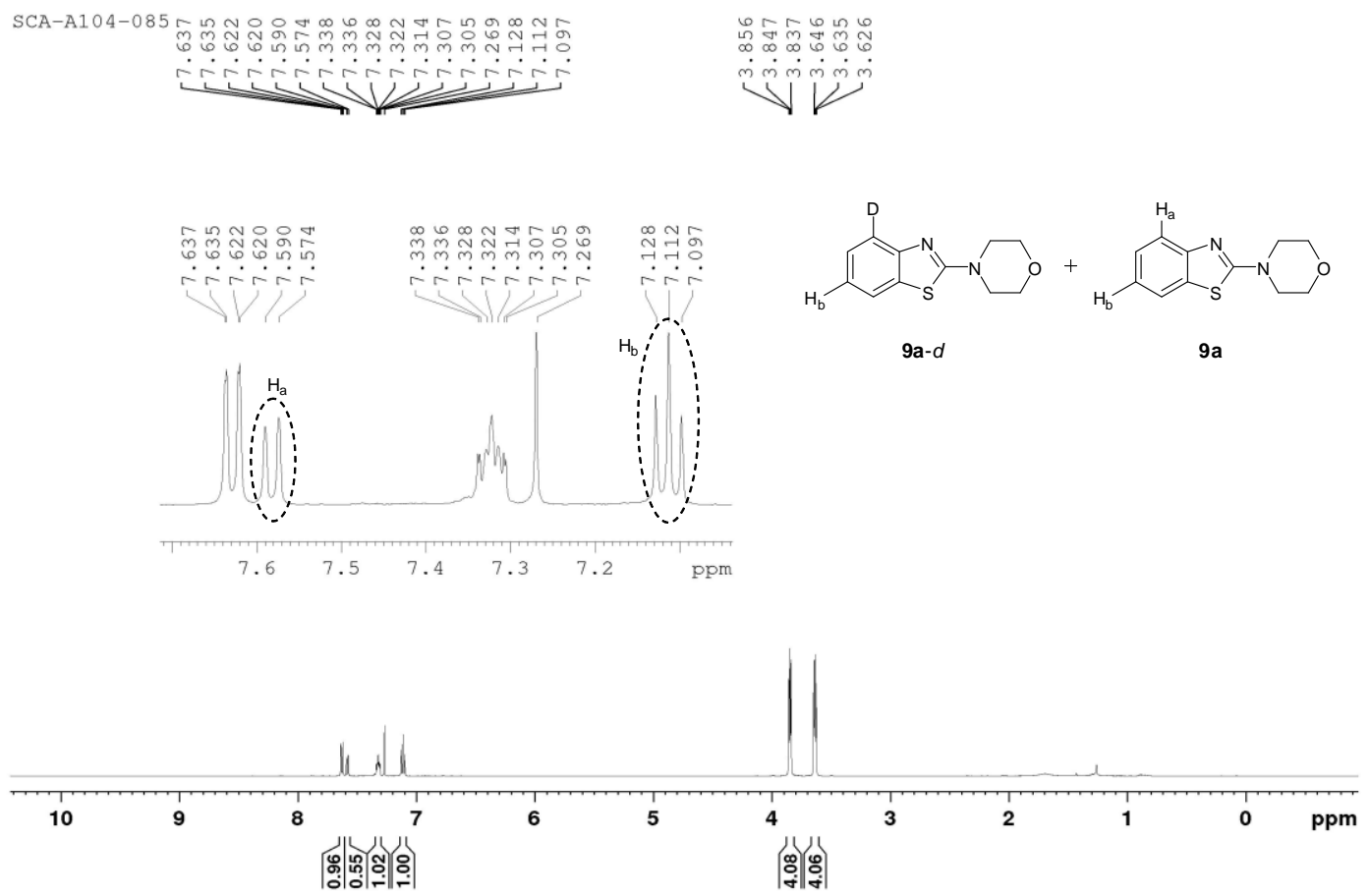

Figure S58: ${ }^{1} \mathrm{H}$ NMR spectra of Kinetic isotopic effect experiment showing mixture of 9a and 9a- $d_{1}$. 


\section{Radical Trap Experiment}<smiles>S=C(Nc1ccccc1)N1CCOCC1</smiles>

$8 a$

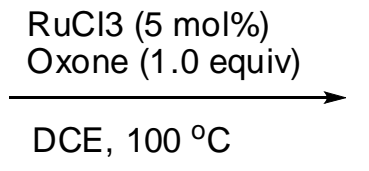

Condition:

None-

TEMPO-

Galvinoxyl-<smiles>c1ccc2sc(N3CCOCC3)nc2c1</smiles>

$9 a$

$87 \%$

$85 \%$

$83 \%$

PROCEDURE: The reactions were been carried out as per the protocol reported in Section 3 in the presence of either TEMPO or Galvinoxyl (free radical scavenger). The product was purified by silica-gel column chromatography (EtOAc: hexane) to produce the isolated yields of 9a.

\section{Table S7: Kinetic Studies}<smiles>[R]c1cccc(NC(=S)N2CCOCC2)c1</smiles><smiles>O=C(c1ccccc1)c1cccc(NC(=S)N2CCOCC2)c1</smiles>

$8 a$<smiles>COc1cccc(NC(=S)N2CCOCC2)c1</smiles>

$8 \mathbf{i}$<smiles>O=[N+]([O-])c1cccc(NC(=S)N2CCOCC2)c1</smiles>

8j

\begin{tabular}{cccc}
\hline Time & $\mathbf{8 a}$ & $\mathbf{8 i}$ & $\mathbf{8 j}$ \\
& $($ Yield\%) & (Yield\%) & (Yield\%) \\
\hline 0 & 0 & 0 & 0 \\
2.5 & 21.86 & 48.9 & 3 \\
0.5 & 46.6 & 75 & 8 \\
1 & 65 & 85 & 10 \\
1.5 & 72 & 87 & 15 \\
2 & 81 & 90 & 28 \\
2.5 & 83 & 91 & 37 \\
3 & 86 & 91 & 54 \\
4 & 87 & 91 & 65 \\
6 & 87 & 91 & 77 \\
8 & 87 & 91 & 77 \\
10 & 87 & 91 & 77 \\
\hline
\end{tabular}




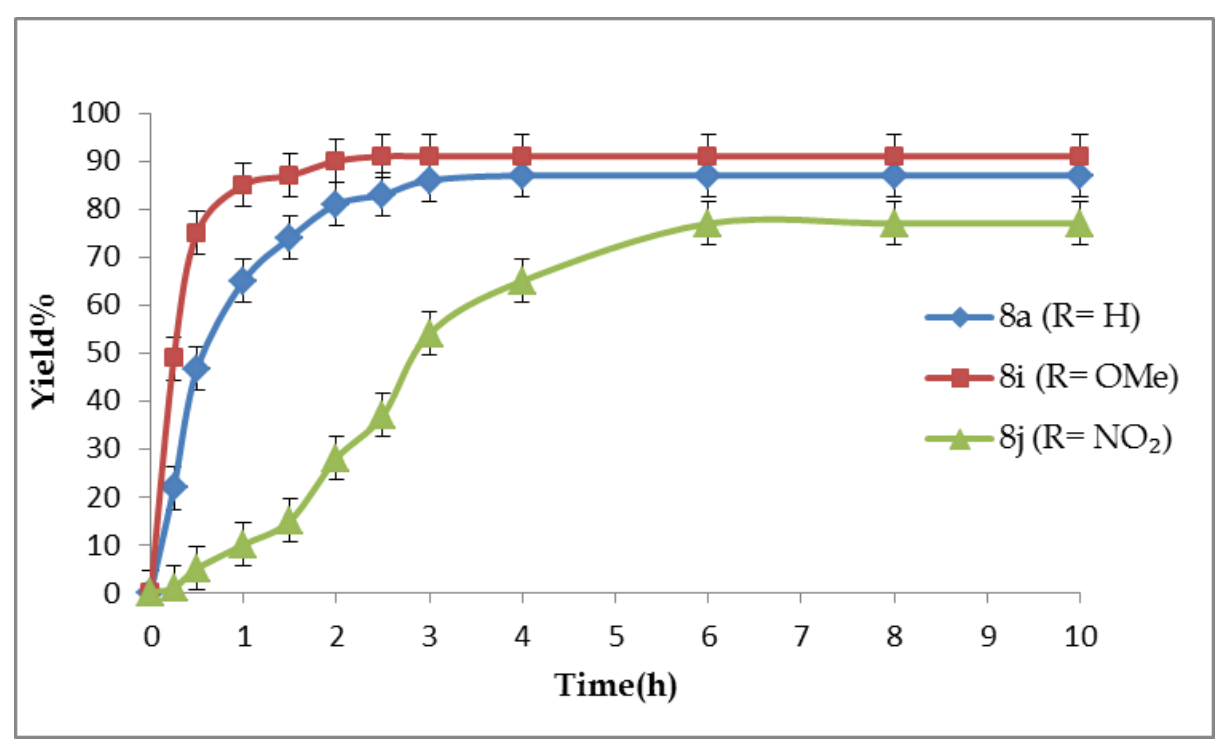

Figure S59: Plot of yield vs. time in the cyclizations of $\mathbf{8 a}, \mathbf{8 i}, \mathbf{8 j}$.

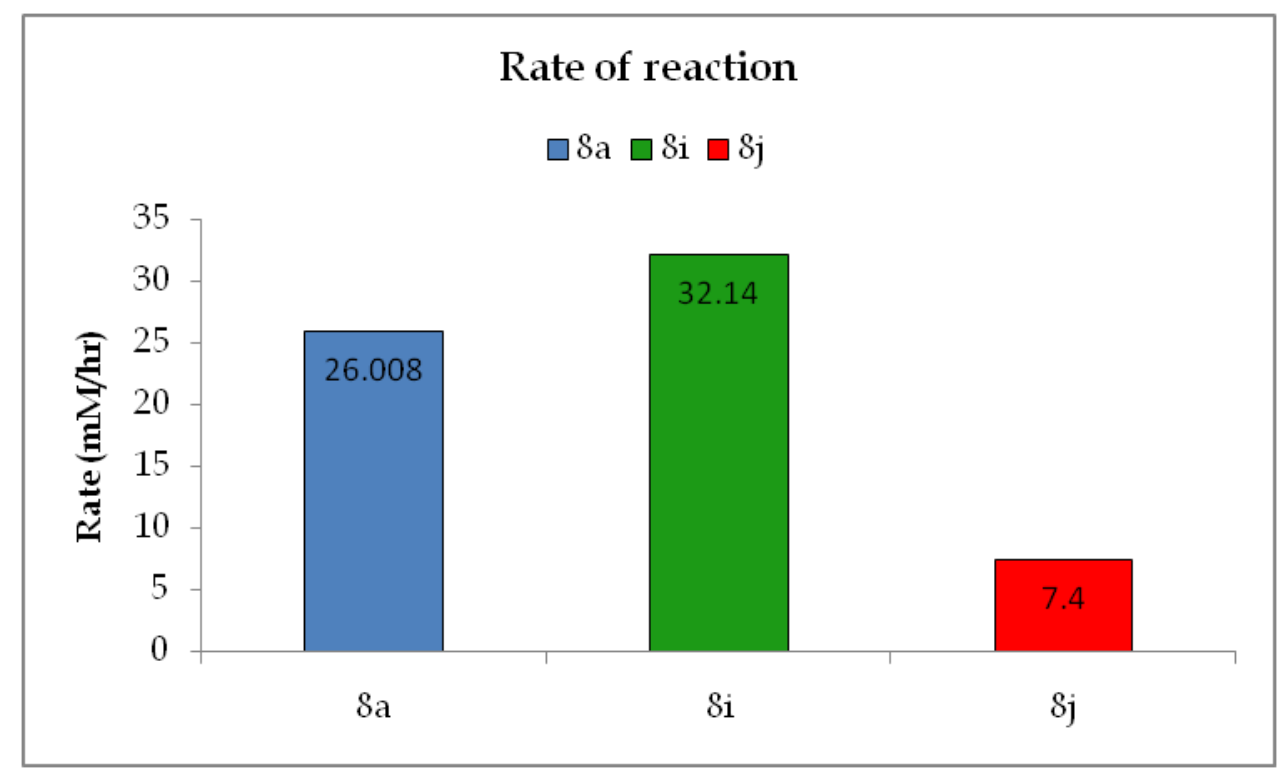

Figure S60: Comparison in the rate of the reaction of $8 \mathrm{a}, 8 \mathrm{i}$ and $8 \mathrm{j}$

\section{Crystallographic Data}

Crystals of 9q and 9u suitable for Single Crystal X-ray Analysis were obtained by slow evaporation in their acetonitrile solutions. Crystal data were collected on Bruker APEX II CCD diffractometer $(\mathrm{MoK} \alpha, \lambda=0.71073 \AA)$. Complete hemispheres of data were collected using $\omega$ scans $\left(0.3^{\circ}\right.$, up to 30 seconds/frame). Integrated intensities were obtained with SAINT ${ }^{7}$ and when they were corrected for absorption SADABS was used. ${ }^{8}$ Structure solution and refinement was performed with the SHELXTL-package. ${ }^{9}$ The structures were solved by direct methods and completed by iterative cycles of DF syntheses and full-matrix least-squares refinement against 
$F^{2} \cdot{ }^{10}$ Non-hydrogen atoms were refined with anisotropic displacement parameters. Hydrogen atoms were fixed at calculated positions and their positions were refined by a riding model. Details of the data collection and refinement parameters are given in Table S7. CCDC 1418622 $(\mathbf{9 q})$ and $1418623(9 \mathbf{u})$ contain the supplementary crystallographic data for this paper. These data can be obtained free of charge at www.ccdc.cam.ac.uk/conts/retrieving.html [or from the Cambridge Crystallographic Data Centre, 12, Union Road, Cambridge CB2 1EZ, UK; fax: +441223/336-033; E-mail: deposit@ccdc.cam.ac.uk].

Table S8: Crystal data and refinement parameters for compounds $9 q$ and $9 u$.

\begin{tabular}{|c|c|c|}
\hline $\begin{array}{l}\text { Compound } \\
\text { Formula }\end{array}$ & $\underset{\mathrm{C}_{13} \mathrm{H}_{10} \mathrm{~N}_{2} \mathrm{~S}}{\mathbf{9 q}}$ & $\begin{array}{c}9 \mathbf{9 u} \\
\mathrm{C}_{14} \mathrm{H}_{12} \mathrm{~N}_{2} \mathrm{OS}\end{array}$ \\
\hline Formula weight & 226.29 & 256.32 \\
\hline $\begin{array}{l}\text { Crystal system } \\
\text { Space group }\end{array}$ & $\begin{array}{l}\text { Triclinic } \\
\text { P-1 }\end{array}$ & $\underset{\mathrm{P}^{\prime} 21 / \mathrm{c}}{\text { Monoclinic }}$ \\
\hline Temperature (K) & $\begin{array}{c}296(2) \\
39105(6)\end{array}$ & $\begin{array}{c}296(2) \\
131514(9)\end{array}$ \\
\hline$b(\AA)$ & $10.8964(17)$ & $12.8014(9)$ \\
\hline$c(\AA)$ & $12.403(2)$ & $7.2224(6)$ \\
\hline$\alpha\left({ }^{\circ}\right)$ & $97.328(3)$ & 90 \\
\hline$\beta\left({ }^{\circ}\right)$ & $94.957(3)$ & $105.094(2)$ \\
\hline$\gamma\left({ }^{\circ}\right)$ & $92.265(3)$ & 90 \\
\hline Volume $\left(\AA^{3}\right)$ & $521.54(14)$ & $1173.99(15)$ \\
\hline$Z$ & 2 & 4 \\
\hline Reflections collected/unique & 3145 & 7716 \\
\hline$R_{\text {int }}$ & 0.0186 & 0.0272 \\
\hline Data/restraints/parameters & $1873 / 0 / 149$ & $2062 / 0 / 168$ \\
\hline Goodness-of-fit on $\mathrm{F}^{2}$ & 1.067 & 1.063 \\
\hline Final $R$ indices $[I>2 \sigma(I)]^{\mathrm{a}}$ & $\begin{aligned} \mathrm{R}_{1} & =0.0311, \\
\mathrm{wR}_{2} & =0.0732\end{aligned}$ & $\begin{array}{l}\mathrm{R}_{1}=0.0299 \\
\mathrm{wR}_{2}=0.0759\end{array}$ \\
\hline$R$ indices (all data) ${ }^{\mathrm{a}}$ & $\begin{array}{c}\mathrm{R}_{1}=0.0369, \\
\mathrm{wR}_{2}=0.0762\end{array}$ & $\begin{aligned} \mathrm{R}_{1} & =0.0350 \\
\mathrm{wR}_{2} & =0.0792\end{aligned}$ \\
\hline Largest residuals $\left(\mathrm{e} . \AA^{-3}\right)$ & $0.19 /-0.29$ & $0.25 /-0.30$ \\
\hline$=\Sigma|| F_{0}|-| F_{c}|| / \Sigma\left|F_{0}\right| ;$ & $\left(\left|F_{0}\right|^{2} \mid F\right.$ & $\left.\left.\left.\left|F_{0}\right|^{2}\right)^{2}\right]\right\}^{1 / 2}$ \\
\hline
\end{tabular}




\section{Computational details}

For all the theoretical investigations the density functional theory (DFT) based Becke threeparameter hybrid (B3) functional was used along with Lee-Yang-Parr (LYP) correction B3LYP formalisms. ${ }^{11}$ The standard $6-31+\mathrm{G}(\mathrm{d}, \mathrm{p})$ basis set ${ }^{12}$ was used to describe the $\mathrm{H}, \mathrm{C}, \mathrm{N}, \mathrm{O}$ and $\mathrm{Cl}$ atoms and Def2-TZVP for $\mathrm{Ru} .{ }^{13}$ The effective core potential variation was used for $\mathrm{Ru}$. The free geometry optimizations for all the species have been performed followed by harmonic vibrational frequencies. Systematic conformational search have been performed to determine the lowest energy conformers for each extrema. Frequency calculations on each equilibriumgeometry show the true minima for reactant and product. Transition state is determined with a vibrational frequency corresponds to the reaction coordinate. Zero-point

energies were included to calculate the activation barrier at room temperature. All the theoretical calculations have been performed using the GAUSSIAN09 package. $^{14}$

\subsection{Cartesian coordinates for intermediate (reactant), transition state and intermediate (product).}

The listed cartesian coordinates correspond to optimizations performed at B3LYP level with Def2-TZVP for Ru and 6-31+G** for all other atoms.

Reactant (12):

$\begin{array}{llll}6 & -0.170850 & -2.500981 & -1.345319 \\ 6 & 0.303306 & -2.186779 & -0.046155 \\ 6 & 1.690389 & -1.901477 & 0.153232 \\ 6 & 2.543793 & -1.883296 & -0.977210 \\ 6 & 2.043526 & -2.163955 & -2.241894 \\ 6 & 0.681747 & -2.475040 & -2.440667 \\ 1 & -1.214597 & -2.781191 & -1.452120 \\ 1 & 3.600353 & -1.687974 & -0.822382 \\ 1 & 2.721207 & -2.163308 & -3.092380 \\ 1 & 0.316649 & -2.718757 & -3.433907 \\ 7 & 2.241133 & -1.767872 & 1.405092 \\ 6 & 1.641468 & -1.184208 & 2.390819\end{array}$




\begin{tabular}{|c|c|c|c|}
\hline 1 & 2.100585 & -1.299543 & 3.375501 \\
\hline 16 & 0.241020 & -0.156938 & 2.451326 \\
\hline 44 & -0.694197 & 0.056515 & 0.255824 \\
\hline 1 & -0.299208 & -2.480624 & 0.806098 \\
\hline 17 & -2.565532 & -1.276780 & 1.038960 \\
\hline 6 & -1.186792 & 0.711002 & -1.865288 \\
\hline 6 & 0.194376 & 0.908021 & -1.541032 \\
\hline 6 & 0.617129 & 1.751292 & -0.474844 \\
\hline 6 & -0.412632 & 2.216856 & 0.394915 \\
\hline 6 & -1.785698 & 1.931819 & 0.149734 \\
\hline 6 & -2.196207 & 1.230305 & -1.034897 \\
\hline 1 & -1.453108 & 0.078023 & -2.704168 \\
\hline 1 & 0.946236 & 0.410771 & -2.146069 \\
\hline 1 & -0.149173 & 2.736801 & 1.308697 \\
\hline 1 & -2.534271 & 2.251796 & 0.866752 \\
\hline 6 & 2.093640 & 2.088042 & -0.313968 \\
\hline 1 & 2.645883 & 1.151347 & -0.462218 \\
\hline 6 & -3.654016 & 0.986002 & -1.304285 \\
\hline 1 & -3.794477 & 0.310831 & -2.151595 \\
\hline 1 & -4.132055 & 0.538702 & -0.428130 \\
\hline 1 & -4.153388 & 1.935968 & -1.529972 \\
\hline 6 & 2.518715 & 3.066305 & -1.432550 \\
\hline 1 & 3.591886 & 3.274118 & -1.362981 \\
\hline 1 & 2.317210 & 2.660507 & -2.429230 \\
\hline 1 & 1.984945 & 4.019716 & -1.341936 \\
\hline
\end{tabular}




$\begin{array}{llll}6 & 2.473269 & 2.650738 & 1.062779 \\ 1 & 3.559042 & 2.778895 & 1.118437 \\ 1 & 2.022636 & 3.636301 & 1.232767 \\ 1 & 2.167121 & 1.984459 & 1.873198\end{array}$

Transition state (13):

\begin{tabular}{llll}
6 & 1.753758 & -0.118680 & -1.855695 \\
6 & 1.362086 & -0.724660 & -0.617512 \\
6 & 2.440697 & -1.141430 & 0.235877 \\
6 & 3.783365 & -0.817308 & -0.100325 \\
6 & 4.089249 & -0.135702 & -1.260817 \\
6 & 3.061105 & 0.191207 & -2.171892 \\
1 & 0.984398 & 0.067367 & -2.598264 \\
1 & 4.561295 & -1.150704 & 0.579334 \\
1 & 5.123055 & 0.103300 & -1.495105 \\
1 & 3.301377 & 0.654767 & -3.124782 \\
7 & 2.331466 & -1.955323 & 1.345993 \\
6 & 1.303584 & -2.081688 & 2.104686 \\
1 & 1.352843 & -2.849651 & 2.879034 \\
16 & -0.177057 & -1.157173 & 2.167916 \\
44 & -0.525314 & -0.059062 & 0.200133 \\
1 & 0.472893 & -1.734813 & -0.943587 \\
17 & -0.311645 & -3.003865 & -1.611807 \\
6 & -1.845229 & 0.374464 & -1.546019 \\
6 & -1.005565 & 1.493136 & -1.378433 \\
6 & -0.827753 & 2.096813 & -0.097599 \\
\hline & & & \\
\hline 6 & & & \\
\hline & & &
\end{tabular}




\begin{tabular}{llll}
6 & -1.673721 & 1.640783 & 0.970929 \\
6 & -2.589470 & 0.583059 & 0.781471 \\
6 & -2.626870 & -0.131436 & -0.453499 \\
1 & -1.837932 & -0.175416 & -2.481555 \\
1 & -0.374224 & 1.815281 & -2.198623 \\
1 & -1.556378 & 2.062029 & 1.962854 \\
1 & -3.150244 & 0.204353 & 1.629755 \\
6 & 0.141946 & 3.257111 & 0.067667 \\
1 & 0.924179 & 3.117001 & -0.688842 \\
6 & -3.500060 & -1.344002 & -0.635942 \\
1 & -2.979897 & -2.103407 & -1.224700 \\
1 & -3.775029 & -1.781473 & 0.326753 \\
1 & -4.421236 & -1.057342 & -1.159672 \\
6 & -0.590232 & 4.581379 & -0.243725 \\
1 & 0.111140 & 5.420872 & -0.193706 \\
1 & -1.037535 & 4.572312 & -1.243161 \\
1 & -1.389812 & 4.767369 & 0.482759 \\
1 & 0.827791 & 3.314796 & 1.441637 \\
1 & 1.589906 & 4.100455 & 1.438370 \\
1 & 0.119884 & 3.556731 & 2.242634 \\
\hline 1 & 1.314813 & 2.367060 & 1.687405 \\
\hline & & & \\
\hline & & & \\
1 & & &
\end{tabular}

Product (14):
6
$\begin{array}{lll}1.458595 & 0.830954 & -1.749723\end{array}$
$\begin{array}{llll}6 & 1.208364 & 0.788976 & -0.340368\end{array}$
$\begin{array}{lllll}6 & 2.133820 & 1.557257 & 0.450889\end{array}$ 


\begin{tabular}{|c|c|c|c|}
\hline 6 & 3.173536 & 2.305214 & -0.176487 \\
\hline 6 & 3.348041 & 2.320016 & -1.544437 \\
\hline 6 & 2.476012 & 1.560103 & -2.344948 \\
\hline 1 & 0.822626 & 0.255758 & -2.412798 \\
\hline 1 & 3.832155 & 2.863307 & 0.481403 \\
\hline 1 & 4.151272 & 2.898257 & -1.992373 \\
\hline 1 & 2.598895 & 1.536216 & -3.424817 \\
\hline 7 & 2.193770 & 1.689579 & 1.832351 \\
\hline 6 & 1.392389 & 1.164237 & 2.690875 \\
\hline 1 & 1.605410 & 1.363800 & 3.743382 \\
\hline 16 & -0.018298 & 0.171238 & 2.477331 \\
\hline 44 & -0.445923 & -0.272531 & 0.299280 \\
\hline 1 & 2.761428 & -1.104633 & -0.580019 \\
\hline 17 & 3.492956 & -2.165649 & -0.745936 \\
\hline 6 & -0.842161 & -1.602558 & -1.459191 \\
\hline 6 & -1.568917 & -0.410071 & -1.642338 \\
\hline 6 & -2.408191 & 0.106975 & -0.604833 \\
\hline 6 & -2.656112 & -0.733812 & 0.533607 \\
\hline 6 & -1.955888 & -1.935867 & 0.705536 \\
\hline 6 & -0.954873 & -2.341627 & -0.236855 \\
\hline 1 & -0.116902 & -1.918438 & -2.202145 \\
\hline 1 & -1.401391 & 0.194299 & -2.527564 \\
\hline 1 & -3.298784 & -0.379625 & 1.331647 \\
\hline 1 & -2.064135 & -2.490634 & 1.632108 \\
\hline 6 & -3.138422 & 1.426110 & -0.812889 \\
\hline
\end{tabular}




$\begin{array}{llll}1 & -2.519745 & 2.015024 & -1.501791 \\ 6 & -0.149932 & -3.595632 & -0.013339 \\ 1 & 0.829595 & -3.530293 & -0.492337 \\ 1 & 0.003273 & -3.780595 & 1.052853 \\ 1 & -0.682192 & -4.458195 & -0.435535 \\ 6 & -4.495450 & 1.161127 & -1.501603 \\ 1 & -5.000156 & 2.108060 & -1.720619 \\ 1 & -4.371449 & 0.616570 & -2.443554 \\ 1 & -5.154940 & 0.570505 & -0.855030 \\ 6 & -3.320421 & 2.252487 & 0.469778 \\ 1 & -3.749187 & 3.228853 & 0.221529 \\ 1 & -4.006981 & 1.768909 & 1.174255 \\ 1 & -2.366366 & 2.414434 & 0.978898\end{array}$



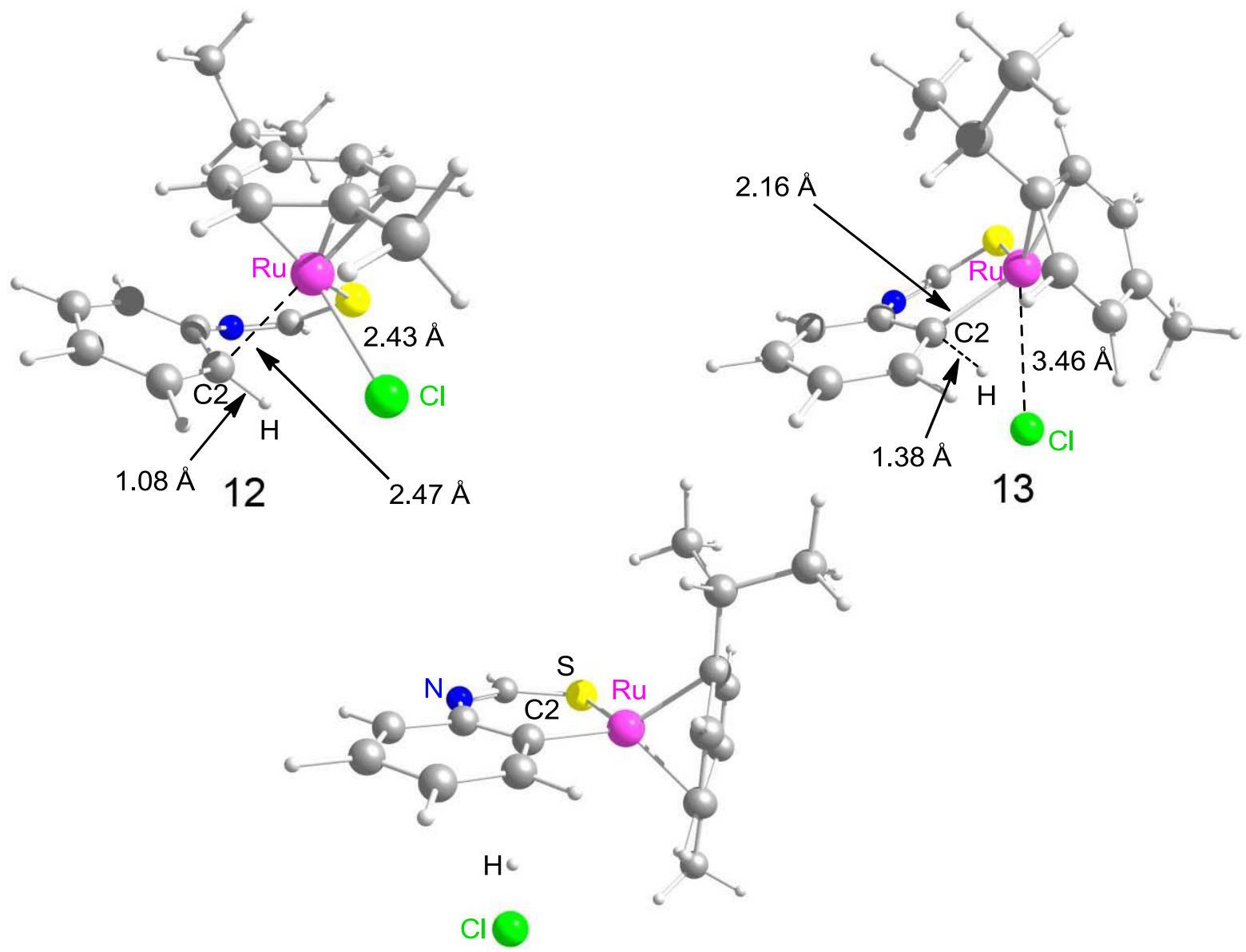

13

Figure S61. Calculated structures of 12, 13 and 14.

\section{Reference}

1] D. Sriram, P. Yogeeswari, K. Madhu, Bioorg. Med.Chem. Lett., 2006, 16, 876-878

2] I. Özdemir, S.Demir, B. Çetinkaya, C. Gourlaouen, F. Maseras, C. Bruneau and P. H. Dixneuf, J. Am. Chem. Soc., 2008, 130, 1156-1157.

3] L. L. Joyce, R. A. Batey, Org. Lett.,2009, 11, 2792-2795.

4] S. K. Sahoo, A. Banerjee, S. Chakraborty and B. K. Patel, ACS Catal., 2012, 2, 544-551.

5] K. Inamoto, C. Hasegawa, J. Kawasaki, K. Hiroya and T. Doi, Adv. Synth. Catal.,2010, 352, 2643-2655.

6] D. Kumar, B. B. Mishra and V. K. TiwariJ. Org. Chem. 2014, 79, 251-266.

7] SMART \& SAINT Software Reference manuals, version 6.45; Bruker Analytical X-ray Systems, Inc.: Madison, WI, 2003. 
8] G. M. Sheldrick, SADABS a software for empirical absorption correction; Version 2.05 University of Göttingen: Göttingen, Germany, 2002.

9] (a) SHELXTL Reference Manual, version 6.1; Bruker Analytical X-ray Systems, Inc.: Madison, WI, 2000. (b) Sheldrick, G. M. SHELXTLv.6.12, Bruker AXS Inc., Madison, WI, 2001.

10] G. M. Sheldrick, SHELXL97, Program for Crystal Structure Refinement; University of Göttingen: Göttingen, Germany, 1997.

11] (a) A. D. Becke, J. Chem. Phys. 1993, 98, 5648-5652. (b) C. Lee, W. Yang, R. Yang and R. G. Parr, Phys. Rev. B 1988, 37, 785-789.

12] (a) T. Clark, J. Chandersekhar, G. W. Spitz-nagel and P. V. R. Schleyer, J. Comput. Chem. 1983, 4, 294-301. (b) M. J. Frisch, J. A. Pople, and J. S. Binkley J. Chem.Phys.1984, 80, 3265-3269.

13] F. Weigend, Phys. Chem. Chem. Phys. 2006, 8, 1057-1065.

14] M. J. Frisch et al., Gaussian 09, revision D.01, Gaussian, Inc. Wallingford CT, 2010. 